\title{
Entropy, Limit Theorems, and Variational Principles for Disordered Lattice Systems
}

\section{Timo Seppäläinen}

Department of Mathematics, Ohio State University, Columbus, OH 43210, USA. E-mail address: timosepp@math.ohio-state.edu.

Received: 4 March 1993/in revised form: 22 August 1994

\begin{abstract}
We study infinite volume limits and Gibbs states of disordered lattice systems with bounded and continuous potentials. Our main tools are a generalization of relative entropy for random reference measures and a large deviation theory for nonstationary independent processes. We find that many familiar results of invariant potentials, such as large deviation theorems, variational principles, and equivalence of ensembles, continue to hold for disordered models, with suitably modified statements.
\end{abstract}

\section{Contents}

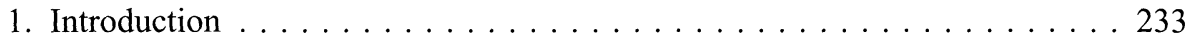

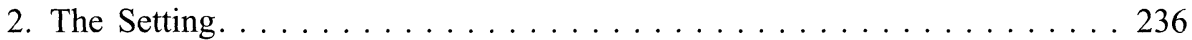

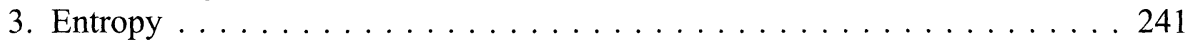

4. The Finite Volume Model . . . . . . . . . . . . . . . . . . . . . . . . 248

5. Thermodynamic Limits. . . . . . . . . . . . . . . . . . . . . 251

6. Infinite Volume Gibbs States . . . . . . . . . . . . . . . . . . . . . . 257

7. Large Deviations . . . . . . . . . . . . . . . . . . . . 260

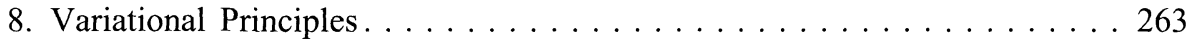

9. Equivalence of Ensembles for Observables . . . . . . . . . . . . . . 266

10. Equivalence of Ensembles for Measures . . . . . . . . . . . . . . 271

\section{Introduction}

This paper studies disordered lattice systems, utilizing some recent large deviation theory for nonstationary processes. Our three interrelated goals are to establish infinite volume limit theorems, to describe Gibbs states by variational principles, and to find the natural entropy functions for these models and study the role of entropy in the limit theorems and variational principles.

Disordered lattice models are interacting spin systems on an integer lattice $\mathbb{Z}^{d}$ whose interaction potential is not necessarily shift invariant. The loss of invariance 
is compensated by making the interaction $\Phi^{\mathbf{x}}$ dependent on an auxiliary parameter $\mathbf{x}$, called the quenched variable, in a way that respects the $\mathbb{Z}^{d}$ action: $\boldsymbol{\Phi}^{\theta_{\mathbf{i}} \mathbf{x}}=\boldsymbol{\Phi}^{\mathbf{x}} \circ \theta_{-\mathbf{i}}$. (Precise definitions follow in Sect. 2.) Thus the mathematical description of the model involves two spaces with $\mathbb{Z}^{d}$ actions, the space $\Omega$ of spin configurations and the space $\mathbf{X}$ of quenched variables. $\mathbb{Z}^{d}$ acts on $\Omega$ by shifts or translations and on $\mathbf{X}$ by some homeomorphisms we need not specify. In Sect. 2 we explain how three familiar models, namely the random field Ising model, the $A_{p} B_{1-p}$-model of a quenched mixed magnetic crystal, and the Edwards-Anderson spin glass model, fit into our framework. We refer the reader to [Z] for a general overview of disordered models and for references to past work.

As usual in equilibrium statistical mechanics, our interest focuses on various aspects of the thermodynamic equilibrium of the spins in the infinite volume limit. The quenched variables do not participate in the thermodynamic equilibrium, but they function as constraints of the equilibrium by specifying an "environment" for the spins: The equilibrium of the spins takes place under a fixed quenched variable $\mathbf{x}$ or under a fixed distribution $\pi$ on $\mathbf{X}$. This situation is called quenched disorder.

We study the setting from two points of view, called the skew model and the sample model. The skew model lives on the product space $\boldsymbol{\Omega}:=\Omega \times \mathbf{X}$ and probability measures describing equilibria are joint distributions of spins and quenched variables. The special role of the quenched variable is reflected in the marginal distribution on $\mathbf{X}$ : Either a point mass $\delta_{\mathbf{x}}$ or a fixed distribution $\pi$, while the spins are either independent under an a priori measure or governed by a Gibbs distribution. The sample model lives on the space $\Omega$, and is obtained from the skew model by projecting all the relevant functions and measures to $\Omega$. Stating results for the skew and sample models separately may seem like an unnecessary duplication, but we shall find that the large deviations and variational principles of the two models have interestingly differing mathematical descriptions.

We restrict to absolutely summable interactions that are continuous functions of the spin and the quenched variable. These are certainly not optimal assumptions for many of our results. But with this restriction we can vary the distribution $\pi$ of the quenched variable freely over the space of all invariant measures on $\mathbf{X}$ and treat the thermodynamic quantities, pressure, entropy, and energy, as functions of $\pi$. We also get deterministic results for fixed quenched variables in addition to $\pi$-almost sure results. And local specifications are well-defined for all configurations, so infinite volume Gibbs states can be defined by DLR-equations. In contrast, other treatments typically fix an i.i.d. distribution $\pi$ on the quenched variables and prove results for a.e. $\mathbf{x}$. The advantage of this approach is that the interaction need not be uniformly bounded, but only sufficiently integrable under $\pi$. No doubt many of our results continue to hold under such assumptions.

Next a brief overview of the contents. Section 2 describes the setting. Section 3 introduces a generalization of relative entropy suitable for handling situations where the reference measure is random. This entropy function is useful for the large deviation theory of nonstationary, independent processes, which we summarize in Sect. 3 for later use. In Sect. 4 we characterize Gibbs states of finite volume skew and sample models in terms of variational principles, paving the way for the infinite volume variational principles of Sect. 8 .

Section 5 moves from finite to infinite volume considerations. We prove the existence of the thermodynamic limit of the pressure. (The remark on p. 180 of [Gr] is in order here, namely that pressure may have to be interpreted as free energy, depending on the model. We follow [Is] and [Z] and call this quantity pressure.) 
The pressure is a function of the interaction and the quenched variable. Via the idea of variables generic for a measure we can treat the pressure also as a function of the distribution $\pi$ of the quenched variable. Additionally, we prove the thermodynamic limit for a function that formally assumes the role of energy in the finite volume variational principle of the sample model. In Sect. 8 we see that this limit takes on the role of specific energy in the infinite volume variational principle.

Section 6 defines various classes of measures and measurable families of measures on $\boldsymbol{\Omega}$ and $\Omega$ that satisfy DLR-equations and hence are Gibbs states of the infinite volume models. Section 7 studies the large deviations of empirical measures under conditioned skew Gibbs states and under sample Gibbs states. The rate functions are expressed in terms of entropies relative to Gibbs kernels. In Sect. 8 we show that infinite volume Gibbs states satisfy variational principles. Sections 9 and 10 apply the large deviation theory to derive equivalence of ensembles results. In Sect. 9 we repeat the results of [La] for observables. In Sect. 10 we look at equivalence of ensembles at the level of measures, seeking results that parallel those of [DSZ] and [Ge2] for invariant potentials.

Throughout the paper we find that results familiar from the invariant theory continue to hold for the skew model, often with only small changes. The sample model picture is not as complete: Under large deviations and variational principles our results cover the sample model as well as the skew model. However, since the Hamiltonian is a function of both the spin and the quenched variable, it is not clear what "conditioning on an energy surface" might mean in the sample model, hence our equivalence of ensembles results are worked out in the skew model setting.

The obvious shortcoming of our results is that they are very general and softanalytic in nature. Explicit computations for interesting statistical mechanical models are often much harder than general theorems, and it remains to be seen whether our approach can say anything interesting about particular models. For some disorderd mean-field models large deviation techniques can yield exact numerical results: In [Se4] this approach is used to rigorously calculate critical exponents of a CurieWeiss version of the $A_{p} B_{1-p}$-model. A mean-field model is more amenable to large deviation techniques because its Hamiltonian is a function of the empirical distribution (a "level 2" object in the Donsker-Varadhan large deviation theory) whereas the Hamiltonian of a Gibbsian model with an interaction potential is a function of the empirical field (a level 3 object), and level 2 rate functions are much easier to calculate explicitly than level 3 rate functions.

The use of large deviation theory in the statistical mechanics of invariant potentials has been well-established since the pioneering work of Lanford [La]. Further examples of this work and references can be found in [Com1, DSZ, E, FO, Ge2, O1, Str, and SZ]. An early use of large deviation theory to study spin glasses was made in [HEC]. Their mean-field-type Hamiltonian can be formulated as a function of order parameters to take advantage of the i.i.d. large deviation theory of the spins under the a priori measure. But to develop large deviation techniques for spin glasses that yield the same results as the tools for invariant statistical mechanics requires a large deviation theory for nonstationary processes. Such results were first presented in [Com2] for i.i.d. quenched variables. The reader will find numerous physical examples described in [Com2], whose assumptions of continuity and boundedness of the interaction are the same as ours. The idea of generic variables appears in [Le] in a proof of the thermodynamic limit of a random Ising model. A recent related paper is $[Z]$, which also lays down elements of a theoretical framework for disordered lattice systems. With techniques completely different from ours it proves 
thermodynamic limits and variational principles for interactions that are unbounded but integrable under the distribution of the quenched variable. In Sect. 8 we connect our variational principle with that of $[\mathrm{Z}]$.

The emerging theory of Markov chains with random transitions (MCRT), also called Markov chains in random environments, deserves to be pointed out to the reader here, though we will not pursue this direction in the present paper. But there is an obvious connection between MCRT's and noninvariant interactions, just as between time-homogeneous Markov chains and invariant potentials (for the latter, see [Ge1]). [Cog1-Cog3 and Or2] have studied MCRT's and contain numerous references to earlier work. A large deviation theory for MCRT's is developed in [Se3].

\section{The Setting}

Let $\mathbb{Z}^{d}$ be the $d$-dimensional integer lattice, and $\Omega:=\mathscr{S} \mathbb{Z}^{d}$ a configuration space with spins in a Polish space $\mathscr{S} . \mathbb{Z}^{d}$ carries the $\ell^{1}$-topology normed by $|\mathbf{i}|:=\left|\mathbf{i}_{1}\right|+$ $\cdots+\left|\mathbf{i}_{d}\right|$. The shift maps $\theta_{\mathbf{i}}$ on $\Omega$ are defined by $\left(\theta_{\mathbf{i}} \sigma\right)_{\mathbf{j}}=\sigma_{\mathbf{i}+\mathbf{j}}$ for $\mathbf{i}, \mathbf{j} \in \mathbb{Z}^{d}$ and $\sigma \in \Omega$. For finite $\Lambda \subset \mathbb{Z}^{d}$, write $\Lambda \subset \subset \mathbb{Z}^{d}$, and define finite volume configuration spaces $\Omega_{\Lambda}:=\mathscr{S}^{\Lambda} . \mathscr{R}$ stands for the class of finite rectangles in $\mathbb{Z}^{d}$.

The space $\mathbf{X}$ of quenched variables is another Polish space, equipped with a continuous $\mathbb{Z}^{d}$ action: there is a group of homeomorphisms $\left\{\theta_{\mathbf{i}}: \mathbf{i} \in \mathbb{Z}^{d}\right\}$ on $\mathbf{X}$ satisfying $\theta_{\mathbf{i}} \circ \theta_{\mathbf{j}}=\theta_{\mathbf{i}+\mathbf{j}}$. Set $\mathscr{S}=\mathscr{S} \times \mathbf{X}, \boldsymbol{\Omega}_{\Lambda}=\Omega_{\Lambda} \times \mathbf{X}$, and $\boldsymbol{\Omega}=\Omega \times \mathbf{X}$. The $\mathbb{Z}^{d}$ action on $\boldsymbol{\Omega}$ is given by $\boldsymbol{\theta}_{\mathbf{i}}(\sigma, \mathbf{x})=\left(\theta_{\mathbf{i}} \sigma, \theta_{\mathbf{i}} \mathbf{x}\right)$. As a rule, boldface notation will distinguish the skew model from the sample model.

The Borel fields of $\mathscr{S}$ and $\mathbf{X}$ are denoted by $\mathscr{B}_{\mathscr{S}}$ and $\mathscr{B}_{\mathbf{X}}$, respectively. $\mathscr{F}_{\Lambda}$ is the $\sigma$-field generated by the spins $\left(\sigma_{\mathbf{i}}: \mathbf{i} \in \Lambda\right)$, and $\mathscr{F}_{\Lambda}:=\mathscr{F}_{\Lambda} \vee \mathscr{B}_{\mathbf{X}} \cdot C_{b}(\Omega)$ denotes the Banach space of bounded continuous functions on $\Omega$ under the supremum norm $\|\cdot\| \cdot \mathscr{C}_{\Lambda}$ is the subspace of $\mathscr{F}_{\Lambda}$-measurable functions in $C_{b}(\Omega)$. The class $\mathscr{C}$ of local functions is the union of the $\mathscr{C}_{\Lambda}$ over $\Lambda \subset \subset \mathbb{Z}^{d}$. Similarly on $\boldsymbol{\Omega}$ we have the classes $\mathscr{C}_{\Lambda}$ of $\mathscr{F}_{\Lambda}$-measurable $C_{b}(\boldsymbol{\Omega})$-functions and their union $\mathscr{C}$. The uniform closure $\overline{\mathscr{C}}$ of $\mathscr{C}$ is a Banach subspace of $C_{b}(\boldsymbol{\Omega})$. Note that while $\mathbf{X}$ may also be an infinite product space, a $\mathscr{C}$-function need not be local in the $\mathbf{x}$-variable.

$\mathscr{M}_{1}(\mathscr{S})$ denotes the space of Borel probability measures on $\mathscr{S}$. Spaces of probability measures are always endowed with their weak topologies, generated by bounded continuous functions. It is important to note that the weak topologies of $\mathscr{M}_{1}(\Omega)$ and $\mathscr{M}_{1}(\boldsymbol{\Omega})$ are also generated by the classes $\mathscr{C}$ and $\mathscr{C}$, respectively.

For each $\mathbf{x} \in \mathbf{X}$, assume given a probability measure $\lambda_{o}^{\mathbf{x}}$ on $\mathscr{S}$ so that the integral

$$
\lambda_{o}^{\mathbf{x}}(f)=\int f d \lambda_{o}^{\mathbf{x}}
$$

is a continuous function of $\mathbf{x}$, for each $f \in C_{b}(\mathscr{S})$. $\lambda_{o}^{\mathbf{x}}$ is interpreted as the a priori measure of the spin at site $\mathbf{0}$, in the sample specified by the quenched variable $\mathbf{x}$. In view of the $\mathbb{Z}^{d}$ action, a natural way to extend $\lambda_{o}^{\mathbf{x}}$ to $\Omega$ is

$$
\lambda^{\mathbf{x}}(d \sigma):=\underset{\mathbf{i} \in \mathbb{Z}^{d}}{\otimes} \lambda_{o}^{\theta_{\mathbf{i}} \mathbf{x}}\left(d \sigma_{\mathbf{i}}\right)
$$

so the spins are independent under $\lambda^{\mathbf{x}}$ and the distribution of $\sigma_{\mathbf{i}}$ is $\lambda_{o}^{\theta_{\mathbf{i}} \mathbf{x}}$. The a priori measure on $\boldsymbol{\Omega}$ is $\lambda^{\mathbf{x}}:=\lambda^{\mathbf{x}} \otimes \delta_{\mathbf{x}}$. Restrictions to finite volume configuration spaces are denoted by 


$$
\lambda_{\Lambda}^{\mathbf{x}}\left(d \sigma_{\Lambda}\right):=\otimes_{\mathbf{i} \in \Lambda}^{\otimes} \lambda_{o}^{\theta_{\mathbf{i}} \mathbf{x}}\left(d \sigma_{\mathbf{i}}\right)
$$

and $\lambda_{\Lambda}^{\mathbf{x}}:=\lambda_{\Lambda}^{\mathbf{x}} \otimes \delta_{\mathbf{x}}$. The continuity of the integrals ensures that these measures are continuous functions of $\mathbf{x}$ in the weak topologies. We shall leave out the superscript " $x$ " to indicate that the quenched variable has been integrated out, as for example in $\lambda:=\int \lambda^{\mathbf{x}} \pi(d \mathbf{x})$.

2.1. Definition. An absolutely summable, continuous, invariant interaction potential on $\boldsymbol{\Omega}$ is a collection $\boldsymbol{\Phi}=\left\{\Phi_{A}: A \subset \subset \mathbb{Z}^{d}\right\} \subset \mathscr{C}$ of bounded local functions satisfying

(a) Each $\Phi_{A}$ is $\mathscr{F}_{A}$-measurable.

(b) $\Phi_{A} \circ \theta_{\mathbf{i}}=\Phi_{\mathbf{i}+A}$ for $A \subset \subset \mathbb{Z}^{d}$ and $\mathbf{i} \in \mathbb{Z}^{d}$.

(c) $\|\boldsymbol{\Phi}\|:=\sum_{A: A \ni 0}\left\|\Phi_{A}\right\|<\infty$.

Let $\mathbb{B}$ denote the space of such objects $\boldsymbol{\Phi}$. $\mathbb{B}$ is a Banach space under the norm $\||\cdot|\|$.

Let $\Lambda \subset \subset \mathbb{Z}^{d}$. The Hamiltonian

$$
H_{\Lambda}:=\sum_{A: A \cap A \neq \emptyset} \Phi_{A}
$$

is a function in $\overline{\mathscr{C}} . \mathbf{G}_{\Lambda}$ is the probability kernel from $\left(\boldsymbol{\Omega}, \mathscr{F}_{\Lambda^{c}}\right)$ into $(\boldsymbol{\Omega}, \mathscr{F})$ that acts on bounded Borel functions $f$ on $\boldsymbol{\Omega}$ by

$$
\mathbf{G}_{\Lambda}^{\sigma, \mathbf{x}}(f):=\frac{1}{Z_{\Lambda}^{\sigma, \mathbf{x}}} \int_{\boldsymbol{\Omega}} f e^{-H_{\Lambda}} d \boldsymbol{\lambda}_{\Lambda}^{\mathbf{x}} \otimes \delta_{\sigma_{\Lambda} c}
$$

with the partition function

$$
Z_{\Lambda}^{\sigma, \mathbf{x}}:=\int_{\boldsymbol{\Omega}} e^{-H_{\Lambda}} d \lambda_{\Lambda}^{\mathbf{x}} \otimes \delta_{\sigma_{\Lambda}^{c}}
$$

It is clear from the formula that $\mathbf{G}_{A}^{\sigma, \mathbf{x}}$ is a continuous measure-valued function of $\left(\sigma_{\Lambda^{c}}, \mathbf{x}\right)$. It acts as a continuous map on probability measures $Q \in \mathscr{M}_{1}(\boldsymbol{\Omega})$ by the rule

$$
\left(\mathbf{G}_{\Lambda} Q\right)(f)=\int_{\boldsymbol{\Omega}} \mathbf{G}_{\Lambda}^{\sigma, \mathbf{x}}(f) Q(d \sigma, d \mathbf{x})
$$

A Hamiltonian without an external condition $\sigma_{\Lambda^{c}}$ is defined by

$$
H_{\Lambda}^{0}:=\sum_{A: A \subset A} \Phi_{A}
$$

with the corresponding probability kernels

$$
d \mathbf{G}_{\Lambda}^{0, \mathbf{x}}:=\frac{1}{Z_{\Lambda}^{0, \mathbf{x}}} e^{-H_{A}^{0}} d \lambda^{\mathbf{x}} .
$$

$\mathbf{G}_{\Lambda}^{0}: \mathbf{X} \mapsto \mathbf{G}_{\Lambda}^{0, \mathbf{x}}$ is a continuous map from $\mathbf{X}$ into $\mathscr{M}_{1}(\boldsymbol{\Omega})$.

The sample model is obtained by considering $\mathbf{x}$-sections of functions $f$ on $\boldsymbol{\Omega}$, defined by $f^{\mathbf{x}}(\sigma):=f(\sigma, \mathbf{x})$. Thus each $\boldsymbol{\Phi} \in \mathbb{B}$ and $\mathbf{x} \in \mathbf{X}$ give a potential

$$
\boldsymbol{\Phi}^{\mathbf{x}}:=\left\{\Phi_{A}^{\mathbf{x}}: A \subset \subset \mathbb{Z}^{d}\right\}
$$


on $\Omega$, no longer necessarily invariant. The Hamiltonians are $H_{\Lambda}^{\mathbf{x}}$ and $H_{\Lambda}^{0, \mathbf{x}}$. The partition functions are the same as for the skew model, and the probability kernels $G_{\Lambda}^{\sigma, \mathbf{x}}, \sigma \in \Omega \cup\{0\}$, are the $\Omega$-marginals of the corresponding skew kernels $\mathbf{G}_{\Lambda}^{\sigma, \mathbf{x}}$. Note that $\mathbf{x}$ remains constant when the kernels of the sample model act on measures:

$$
\left(G_{\Lambda}^{\mathbf{x}} \mu\right)(f)=\int_{\Omega} G_{\Lambda}^{\sigma, \mathbf{x}}(f) \mu(d \sigma)
$$

for $\mu \in \mathscr{M}_{1}(\Omega)$ and bounded Borel functions $f$ on $\Omega$.

The contribution of the spin at site $\mathbf{0}$ is measured by

$$
\varphi:=\sum_{A: A \ni \mathbf{o}} \frac{\Phi_{A}}{|A|} .
$$

The assumption $\|\boldsymbol{\Phi}\| \mid<\infty$ guarantees that $\varphi \in \overline{\mathscr{C}}$. Throughout the paper, we take infinite volume limits along the fixed sequence of cubes

$$
V_{n}:=[-n, n]^{d} \cap \mathbb{Z}^{d} .
$$

To simplify the notation, the subscript $V_{n}$ is replaced by $n$, as in $H_{n}=H_{V_{n}}$.

Invariance and ergodicity under the $\mathbb{Z}^{d}$ action on $\mathbf{X}$ play a major role in our development. Write $\mathscr{M}_{\Theta}(\mathbf{X})$ for the space of invariant Borel probabilities on $\mathbf{X}$. Call a quenched variable $\mathbf{x}$ generic for $\pi$, if $\pi \in \mathscr{M}_{1}(\mathbf{X})$ and

$$
\lim _{n \rightarrow \infty} \frac{1}{\left|V_{n}\right|} \sum_{i \in V_{n}} \delta_{\theta_{\mathbf{i}} \mathbf{x}}=\pi
$$

in the weak topology of $\mathscr{M}_{1}(\mathbf{X})$. It follows that $\pi \in \mathscr{M}_{\Theta}(\mathbf{X})$. Say $\mathbf{x}$ is generic if the above holds for some (unspecified) $\pi$. By the ergodic theorem,

$$
\pi\{\mathbf{x}: \mathbf{x} \text { is generic }\}=1
$$

for all $\pi \in \mathscr{M}_{\Theta}(\mathbf{X})$. In particular, $\pi \in \mathscr{M}_{\Theta}(\mathbf{X})$ is ergodic if and only if

$$
\pi\{\mathbf{x}: \mathbf{x} \text { is generic for } \pi\}=1 .
$$

When working with a fixed $\pi \in \mathscr{M}_{1}(\mathbf{X})$, we write $\mathscr{M}_{1}^{\pi}(\boldsymbol{\Omega})$ for the set of probability measures on $\boldsymbol{\Omega}$ with $\mathrm{X}$-marginal $\pi$. $\mathscr{M}_{\Theta}^{\pi}(\boldsymbol{\Omega}):=\mathscr{M}_{1}^{\pi}(\boldsymbol{\Omega}) \cap \mathscr{M}_{\Theta}(\boldsymbol{\Omega})$ is the subclass of invariant probability measures.

Empirical measures are defined as follows: The empirical distributions are

$$
L_{n}(\sigma)=\frac{1}{\left|V_{n}\right|} \sum_{\mathbf{i} \in V_{n}} \delta_{\sigma_{\mathbf{i}}} \quad \text { and } \quad \mathbf{L}_{n}(\sigma, \mathbf{x})=\frac{1}{\left|V_{n}\right|} \sum_{\mathbf{i} \in V_{n}} \delta_{\left(\sigma_{\mathbf{i}}, \theta_{\mathbf{i}} \mathbf{x}\right)}
$$

for the sample and skew models, respectively. The empirical fields are

$$
R_{n}(\sigma)=\frac{1}{\left|V_{n}\right|} \sum_{\mathbf{i} \in V_{n}} \delta_{\theta_{\mathbf{i}} \sigma} \quad \text { and } \quad \mathbf{R}_{n}(\sigma, \mathbf{x})=\frac{1}{\left|V_{n}\right|} \sum_{\mathbf{i} \in V_{n}} \delta_{\theta \mathbf{i}}(\sigma, \mathbf{x})
$$

The results of this paper rest on a Donsker-Varadhan type large deviation theory for independent but nonstationary random fields. To remind the reader, here is the conventional form of the large deviation principle (see [DS] or [V]): Suppose 
$\xi_{n}$ are random variables with values in a Polish space $\mathscr{U}$. We say that the distributions $P\left\{\xi_{n} \in \cdot\right\}$ on $\mathscr{U}$ satisfy a large deviation principle with rate $I$ if these two requirements are met:

(a) $I$ is a lower semicontinuous function from $\mathscr{U}$ into $[0, \infty]$ with compact sublevel sets $\{I \leqq c\}$ for all real $c$.

(b) For Borel subsets $A$ of $\mathscr{U}$,

$$
\begin{aligned}
-\inf _{A^{\circ}} I & \leqq \liminf _{n \rightarrow \infty} \frac{1}{\left|V_{n}\right|} \log P\left\{\xi_{n} \in A\right\} \\
& \leqq \limsup _{n \rightarrow \infty} \frac{1}{\left|V_{n}\right|} \log P\left\{\xi_{n} \in A\right\} \leqq-\inf _{\bar{A}} I,
\end{aligned}
$$

where $A^{\circ}$ and $\bar{A}$ are the interior and the closure of $A$, respectively.

In our applications $\xi_{n}$ will be an empirical measure and $\mathscr{U}$ the appropriate space of probability measures.

We conclude this section by describing how three familiar disordered models fit the setting described here. We give only the simplest versions of the models with spins that take values in the space $\mathscr{S}=\{-1,+1\}$, as generalizations are easy to write down.

2.3. Example. The random field Ising model (RFIM) with nearest-neighbor interactions can be given in terms of the Hamiltonian

$$
H_{\Lambda}^{0, \mathbf{x}}(\sigma)=-J \sum_{\substack{\{\mathbf{i}, \mathbf{j}\} \subset \Lambda \\|\mathbf{i}-\mathbf{j}|=1}} \sigma_{\mathbf{i}} \sigma_{\mathbf{j}}-\sum_{\mathbf{i} \in \Lambda} h_{\mathbf{i}} \sigma_{\mathbf{i}}
$$

together with the a priori measure

$$
\zeta=\left(\frac{1}{2} \delta_{-1}+\frac{1}{2} \delta_{+1}\right)^{\otimes \mathbb{Z}^{d}} .
$$

The disordered magnetic field variables make up the quenched variable $\mathbf{x}=\left(h_{\mathbf{i}}\right.$ : $\mathbf{i} \in \mathbb{Z}^{d}$ ). However, requirement (c) of Definition 2.1 of an interaction would force us to bound the $h_{\mathbf{i}}$ uniformly. The way around this requirement is to subsume the disorder into the a priori measure. Thus we shall describe the RFIM by taking

$$
\lambda_{o}^{\mathbf{x}}=\frac{1}{e^{-h_{\mathbf{0}}}+e^{h_{\mathbf{0}}}}\left(e^{-h_{\mathbf{0}}} \delta_{-1}+e^{h_{\mathbf{0}}} \delta_{+1}\right)
$$

and superimposing on $\lambda^{\mathbf{x}}$ an ordinary Ising Hamiltonian, namely the first term of (2.4). See $[A W]$ and $[B K]$ for recent rigorous work on this model.

2.7. Example. The $A_{p} B_{1-p}$-model describes the situation where 2 different types of atoms, $\mathrm{A}$ and $\mathrm{B}$, interact on the lattice. The quenched variable $\mathbf{x}=\left(\mathbf{x}_{\mathbf{i}}: \mathbf{i} \in \mathbb{Z}^{d}\right)$ comes from setting $\mathbf{x}_{\mathbf{i}}=1$ or 0 according to whether site $\mathbf{i}$ is occupied by an A- or a B-atom. The measure $\pi$ on $\mathbf{X}=\{0,1\}^{\mathbb{Z}^{d}}$ describes the random mechanism that determines the occupations $\mathbf{x}_{\mathbf{i}}$. This situation is often called site disorder. The point is that the model can simultaneously contain opposing types of magnetic ordering, ferromagnetic and antiferromagnetic, depending on the choices of the coupling constants $J_{A A}, J_{B B}$, and $J_{A B}$ for the three different possible pairs (we are again restricting to nearest-neighbor interactions). The Hamiltonian is given by 
$H_{\Lambda}^{0, \mathbf{x}}(\sigma)=\sum_{\substack{\{\mathbf{i}, \mathbf{j}\} \subset A \\|\mathbf{i}-\mathbf{j}|=1}} \sigma_{\mathbf{i}} \sigma_{\mathbf{j}}\left[J_{A A} \mathbf{x}_{\mathbf{i}} \mathbf{x}_{\mathbf{j}}+J_{B B}\left(1-\mathbf{x}_{\mathbf{i}}\right)\left(1-\mathbf{x}_{\mathbf{j}}\right)+J_{A B}\left(\mathbf{x}_{\mathbf{i}}\left(1-\mathbf{x}_{\mathbf{j}}\right)+\mathbf{x}_{\mathbf{j}}\left(1-\mathbf{x}_{\mathbf{i}}\right)\right)\right]$

and the a priori measure is that of (2.5). See [Ah] for physical background.

2.8. Example. The Edwards-Anderson spin glass model (EA) is an Ising model with random coupling constants. Its Hamiltonian is

$$
H_{\Lambda}^{0, \mathbf{x}}(\sigma)=\sum_{\substack{\{\mathbf{i}, \mathbf{j}\} \subset A \\|\mathbf{i}-\mathbf{j}| \leqq R}} J_{\{\mathbf{i}, \mathbf{j}\}}^{\mathbf{x}} \sigma_{\mathbf{i}} \sigma_{\mathbf{j}}
$$

For the sake of illustration, we allow a general finite range of interaction $R \geqq 1$. Let

$$
D:=\left\{\mathbf{i} \in \mathbb{Z}^{d}: \mathbf{i}>\mathbf{0},|\mathbf{i}| \leqq R\right\},
$$

where $>$ on $\mathbb{Z}^{d}$ denotes strict lexicographic ordering. To adhere to our boundedness requirement 2.1 (c), we need to pick a number $K>0$ that gives the maximum coupling strength, $J_{\{\mathbf{i}, \mathbf{j}\}}^{\mathbf{x}} \leqq K$. Let $E:=[-K, K]^{D}$ and $\mathbf{X}:=E^{\mathbb{Z}^{d}}$. A quenched variable $\mathbf{x} \in \mathbf{X}$ is a doubly indexed configuration $\mathbf{x}=\left(\mathbf{x}_{\mathbf{k}, \mathbf{k}+\mathbf{i}}: \mathbf{k} \in \mathbb{Z}^{d}, \mathbf{i} \in D\right)$ with $-K \leqq \mathbf{x}_{\mathbf{k}, \mathbf{k}+\mathbf{i}} \leqq K$. $\mathbb{Z}^{d}$ acts on $\mathbf{X}$ by $\left(\theta_{\mathbf{j}} \mathbf{x}\right)_{\mathbf{k}, \mathbf{k}+\mathbf{i}}=\mathbf{x}_{\mathbf{k}+\mathbf{j}, \mathbf{k}+\mathbf{i}+\mathbf{j}}$. Set

$$
J_{\{\mathbf{i}, \mathbf{j}\}}^{\mathbf{x}}:= \begin{cases}\mathbf{x}_{\mathbf{i}, \mathbf{j}} & \text { if } \mathbf{j} \in \mathbf{i}+D \\ \mathbf{x}_{\mathbf{j}, \mathbf{i}} & \text { if } \mathbf{i} \in \mathbf{j}+D \\ 0 & \text { otherwise }\end{cases}
$$

Exactly one of the cases happens for each 2-point set $\{\mathbf{i}, \mathbf{j}\}$; this was the point of requiring that $D$ be lexicographically strictly above $\mathbf{0}$. On the other hand, whenever $0<|\mathbf{i}-\mathbf{j}| \leqq R$, then either $\mathbf{j} \in \mathbf{i}+D$ or $\mathbf{i} \in \mathbf{j}+D$. The desired Hamiltonian (2.9) is got by defining the interaction potential as

$$
\Phi_{A}(\sigma, \mathbf{x}):= \begin{cases}J_{\{\mathbf{i}, \mathbf{j}\}}^{\mathbf{x}} \sigma_{\mathbf{i}} \sigma_{\mathbf{j}} & \text { if } A=\{\mathbf{i}, \mathbf{j}\} \\ 0 & \text { if }|A| \neq 2\end{cases}
$$

This arrangement allows nonstationary correlations among some coupling constants: The law of the variable $\mathbf{x}_{\mathbf{k}}=\left(\mathbf{x}_{\mathbf{k}, \mathbf{k}+\mathbf{i}}\right)_{\mathbf{i} \in D}$ can be any probability measure on $E$, while the $E$-valued process $\left(\mathbf{x}_{\mathbf{k}}\right)_{\mathbf{k} \in \mathbb{Z}^{d}}$ has to be stationary with law $\pi$. This formulation is general enough to contain Example 2.7 as a special case.

For general background on the physics of spin-glasses and this model in particular, we refer the reader to $[\mathrm{FH}]$.

2.10. Non-Example. The Sherrington-Kirkpatrick spin glass model, with the Hamiltonian

$$
H_{n}(\sigma)=\frac{1}{\sqrt{n}} \sum_{1 \leqq i<j \leqq n} J_{i, j} \sigma_{i} \sigma_{j}
$$

and i.i.d. coupling constants $\left(J_{i, j}\right)_{1 \leqq i<j<\infty}$, does not fit our setting: 


\section{Entropy}

Recall the entropy of a probability measure $\alpha$ relative to another probability measure $\rho$ defined by

$$
H(\alpha \mid \rho)= \begin{cases}\rho\left(\frac{d \alpha}{d \rho} \log \frac{d \alpha}{d \rho}\right) & \text { if } \alpha \ll \rho, \\ \infty & \text { otherwise } .\end{cases}
$$

The relative entropy functional is at the heart of the link between large deviation theory and equilibrium statistical mechanics: In large deviations, $H(\cdot \mid \rho)$ is the rate function in Sanov's theorem for i.i.d. random variables with common law $\rho$ [DS, Theorem 3.2.17], and in statistical mechanics $-H(\alpha \mid \rho)$ is the entropy of $\alpha$ as a state of a system with a priori measure $\rho$ [Is, p. 38]. An alternative variational formula for the entropy is

$$
H(\alpha \mid \rho)=\sup _{f \in C_{b}(\mathscr{S})}\left\{\alpha(f)-\log \rho\left(e^{f}\right)\right\}
$$

Now suppose that the probability $\rho$ relative to which entropy is measured in (3.1) is replaced by a random probability measure. To be precise, let $\lambda_{o}^{*}$ denote the $\mathscr{M}_{1}(\mathscr{S})$-valued random variable $\mathbf{x} \mapsto \lambda_{o}^{\mathbf{x}}$, defined on the probability space $\left(\mathbf{X}, \mathscr{B}_{\mathbf{X}}, \pi\right)$. Then (3.2) generalizes naturally in the following fashion:

$$
\widehat{H}\left(\alpha \mid \lambda_{o}, \pi\right):=\sup _{f \in C_{b}(\mathscr{S})}\left\{\alpha(f)-\int \log \lambda_{o}^{\mathbf{x}}\left(e^{f}\right) \pi(d \mathbf{x})\right\}
$$

Facts about this entropy (proofs can be found in [Se1] and [Se2]):

(3.a) $\widehat{H}\left(\alpha \mid \lambda_{o}^{\cdot}, \pi\right) \geqq 0$, with equality if and only if $\alpha=\lambda_{o}:=\int \lambda_{o}^{\mathbf{x}} \pi(d \mathbf{x})$, the expectation of the random measure $\lambda_{o}^{\mathbf{x}}$ under $\pi$. The inequality $\widehat{H}\left(\alpha \mid \lambda_{o}^{*}, \pi\right) \geqq H\left(\alpha \mid \lambda_{o}\right)$ holds for all $\alpha \in \mathscr{M}_{1}(\mathscr{S})$, with equality for all $\alpha \in \mathscr{M}_{1}(\mathscr{S})$ if and only if $\lambda_{o}^{\mathbf{x}}=\lambda_{o} \pi$ a.s.

(3.b) If $\mathbf{x}$ is generic for $\pi$, the distributions $\lambda^{\mathbf{x}}\left\{L_{n} \in \cdot\right\}$ on $\mathscr{M}_{1}(\mathscr{S})$ satisfy a large deviation principle with the convex rate $S^{\pi}(\alpha):=\widehat{H}\left(\alpha \mid \lambda_{o}, \pi\right)$. The new notation $S^{\pi}$ is introduced to reserve the notation $\widehat{H}$ for the general object defined in (3.3), for we will encounter $\widehat{H}$-entropies relative to four different random measures: the a priori measures and the Gibbs measures of both the sample and the skew model.

(3.c) In the skew model, the distributions $\lambda^{\mathbf{x}}\left\{\mathbf{L}_{n} \in \cdot\right\}$ on $\mathscr{M}_{1}(\mathscr{S})$ satisfy a large deviation principle with the rate function $\mathbf{S}^{\pi}(v):=\widehat{H}\left(v \mid \boldsymbol{\lambda}_{o}^{\cdot}, \pi\right)$ defined for $v \in \mathscr{M}_{1}(\mathscr{S})$. In the skew model we have the representation

$$
\mathbf{S}^{\pi}(v)= \begin{cases}\int H\left(v^{\mathbf{x}} \mid \lambda_{o}^{\mathbf{x}}\right) \pi(d \mathbf{x})=H\left(v \mid \lambda_{o}\right) & \text { if the marginal } v_{\mathbf{X}}=\pi \\ \text { otherwise }\end{cases}
$$

Here $v^{\mathbf{x}}$ is a conditional distribution of $v$ on $\mathscr{S}$, given $\mathbf{x} \in \mathbf{X}, \lambda_{o}:=\int \lambda_{o}^{\mathbf{x}} \pi(d \mathbf{x})$, and we used the conditional entropy formula [DS, Lemma 4.4.7]. A contraction principle connects the skew and sample model:

$$
S^{\pi}(\alpha)=\inf _{v \mathscr{S}=\alpha} \mathbf{S}^{\pi}(v)=\inf _{\substack{v \mathscr{y}=\alpha \\ v \mathbf{x}=\pi}} H\left(v \mid \boldsymbol{\lambda}_{o}\right)
$$


Next we look at these entropies on configuration spaces. Suppose $\mu$ and $\gamma$ are invariant probability measures on $\Omega$. Write $\mu_{\Lambda}$ for the restriction to $\mathscr{F}_{\Lambda}$, with the convention $\mu_{n}=\mu_{V_{n}}$. Specific relative entropy is defined by

$$
h(\mu \mid \gamma)=\lim _{n \rightarrow \infty} \frac{1}{\left|V_{n}\right|} H\left(\mu_{n} \mid \gamma_{n}\right)
$$

whenever the limit exists. As is well-known, if $\gamma$ is hypermixing or a Gibbs measure for an absolutely summable invariant potential, then $h(\mu \mid \gamma)$ exists for all $\mu \in \mathscr{M}_{\Theta}(\Omega)$ and governs the large deviations of $R_{n}$ under $\gamma$, see for example [DSZ] and [Ge2].

Suppose now that $\pi$ is invariant and, as above, $\mu \in \mathscr{M}_{\Theta}(\Omega)$. By Theorem 3.19 in $[\mathrm{Se} 2]$ the limit

$$
\widehat{h}\left(\mu \mid \lambda^{\bullet}, \pi\right):=\lim _{n \rightarrow \infty} \frac{1}{\left|V_{n}\right|} \widehat{H}\left(\mu_{n} \mid \lambda_{n}^{\cdot}, \pi\right)
$$

exists and satisfies

$$
\widehat{h}\left(\mu \mid \lambda^{\cdot}, \pi\right)=\sup _{\Lambda \in \mathscr{R}} \frac{1}{|\Lambda|} \widehat{H}\left(\mu_{\Lambda} \mid \lambda_{\Lambda}, \pi\right) .
$$

It is perhaps worthwhile to point out that (3.7)-(3.8) follow from the independence built into $\lambda^{\mathbf{x}}$, so no restrictions on $\pi$ are needed, except for invariance. Furthermore (proofs again in [Se1] and [Se2]):

(3.d) Suppose $\mathbf{x}$ is generic for $\pi$. Then the distributions $\lambda^{\mathbf{x}}\left\{R_{n} \in \cdot\right\}$ on $\mathscr{M}_{1}(\Omega)$ satisfy a large deviation principle with the convex rate function

$$
s^{\pi}(\mu):= \begin{cases}\widehat{h}\left(\mu \mid \lambda^{\circ}, \pi\right) & \text { if } \mu \in \mathscr{M}_{\Theta}(\Omega), \\ \infty & \text { otherwise }\end{cases}
$$

$s^{\pi}(\mu)=0$ if and only if $\mu=\lambda:=\int \lambda^{\mathbf{x}} \pi(d \mathbf{x})$. In case $\pi$ is ergodic, $s$ is affine on $\mathscr{M}_{\Theta}(\Omega)$.

(3.e) Analogous results hold for the skew model: The convex rate function

$$
\mathbf{s}^{\pi}(Q):= \begin{cases}\widehat{h}\left(Q \mid \lambda^{\cdot}, \pi\right) & \text { if } Q \in \mathscr{M}_{\Theta}(\boldsymbol{\Omega}), \\ \infty & \text { otherwise }\end{cases}
$$

governs the large deviations of $\lambda^{\mathbf{x}}\left\{\mathbf{R}_{n} \in \cdot\right\}$ on $\mathscr{M}_{1}(\boldsymbol{\Omega})$. For $Q \in \mathscr{M}_{\Theta}(\boldsymbol{\Omega})$, let $Q_{n}$ be its restriction to $\mathscr{F}_{n}$. The entropy

$$
\mathbf{h}(Q \mid \lambda):=\lim _{n \rightarrow \infty} \frac{1}{\left|V_{n}\right|} H\left(Q_{n} \mid \lambda_{n}\right)
$$

exists for $Q \in \mathscr{M}_{\Theta}^{\pi}(\boldsymbol{\Omega})$, and we have

$$
\mathbf{s}^{\pi}(Q)= \begin{cases}\mathbf{h}(Q \mid \lambda) & \text { if } Q \in \mathscr{M}_{\Theta}^{\pi}(\boldsymbol{\Omega}), \\ \infty & \text { otherwise }\end{cases}
$$

$\mathbf{s}^{\pi}$ is affine on $\mathscr{M}_{\Theta}^{\pi}(\boldsymbol{\Omega})$, and on all of $\mathscr{M}_{\Theta}(\boldsymbol{\Omega})$ in case $\pi$ is ergodic [Se1, Theorem 3.4 and Remark 3.8]. Contraction is again in force:

$$
s^{\pi}(\mu)=\inf _{Q_{\Omega}=\mu} \mathbf{s}^{\pi}(Q)
$$

In the typical case $\mathbf{X}$ is a product space with $\mathbb{Z}^{d}$ action by translations and $\lambda_{o}^{\mathbf{x}}=\lambda_{o}^{\mathbf{x}_{0}}$ depends only on the $\mathbf{x}_{\mathbf{0}}$-coordinate. Then $\mathbf{h}(Q \mid \lambda)$ in (3.10) can be replaced 
by the ordinary specific entropy $h(Q \mid \lambda)$ (To justify this take $U=\{\boldsymbol{0}\}$ in Theorem 3.4 of [Se1]). The difference between $\mathbf{h}(Q \mid \lambda)$ and $h(Q \mid \lambda)$ is that in (3.9) each finite volume entropy involves all $\mathbf{x}$-coordinates, because $\mathscr{\mathscr { P }}_{n}$ contains $\mathscr{B} \mathbf{x}$, whereas in (3.6) only those in the box $V_{n}$.

The difference between the skew and sample models alluded to in the introduction is evident here: As (3.4) and (3.10) show, the familiar relative entropy rates govern large deviations under the skew a priori measure, but for the sample model the new entropy $\widehat{H}$ does not reduce to the old one.

Neither (3.3) nor (3.5) is very useful for computing the entropy $\widehat{H}\left(\alpha \mid \lambda_{o}^{\bullet}, \pi\right)$ unless an obvious maximizing $f$ or minimizing $v$ suggests itself. A special case where this happens is when a "generalized derivative" exists: Say a nonnegative Borel function $\psi$ on $\mathscr{S}$ is a generalized derivative of $\alpha$ if

$$
\alpha(g)=\int \frac{\lambda_{o}^{\mathbf{x}}(g \psi)}{\lambda_{o}^{\mathbf{x}}(\psi)} \pi(d \mathbf{x})
$$

for all bounded Borel functions $g$.

3.13. Lemma. If $\psi$ is a generalized derivative for $\alpha$ in the above sense, then

$$
\widehat{H}\left(\alpha \mid \lambda_{o}^{\cdot}, \pi\right)=\int \lambda_{o}^{\mathbf{x}}\left(\frac{\psi}{\lambda_{o}^{\mathbf{x}}(\psi)} \log \frac{\psi}{\lambda_{o}^{\mathbf{x}}(\psi)}\right) \pi(d \mathbf{x}) .
$$

Note that $\psi$ is not necessarily unique, but the function $\Psi(s, \mathbf{x}):=\psi(s) / \lambda_{o}^{\mathbf{x}}(\psi)$ is $\lambda_{o}$-a.s. unique. In case the measures $\lambda_{o}^{\mathbf{x}}$ are sufficiently close to each other over $\pi$ a.e. $\mathbf{x}$, we can strengthen this lemma to give the entropy $\widehat{H}\left(\alpha \mid \lambda_{o}^{\cdot}, \pi\right)$ an expression analogous to (3.1), with the Radon-Nikodym derivative $d \alpha / d \rho$ replaced by the generalized derivative. As for the degree of closeness among the $\lambda_{o}^{\mathbf{x}}$ required, the following result is sufficient for the three models presented as examples. This can be regarded as a correction to [Se2], where the conclusion of Lemma 3.15 was erroneously believed to always hold.

3.15. Lemma. Assume that $\lambda_{o}^{\mathbf{x}} \ll \lambda_{o}^{\mathbf{y}}$ for $\pi \otimes \pi$-a.e. pair $(\mathbf{x}, \mathbf{y})$ and that

$$
\iint\left\|\log \frac{d \lambda_{o}^{\mathbf{x}}}{d \lambda_{o}^{\mathbf{y}}}\right\|_{L^{\infty}\left(\lambda_{o}^{\mathbf{x}}\right)} \pi(d \mathbf{x}) \pi(d \mathbf{y})<\infty .
$$

Then for all $\alpha \in \mathscr{M}_{1}(\mathscr{S})$, either $\alpha$ has a generalized derivative $\psi$ and $\widehat{H}\left(\alpha \mid \lambda_{o}^{\cdot}, \pi\right)$ is given by (3.14) or $\widehat{H}\left(\alpha \mid \lambda_{o}^{*}, \pi\right)=\infty$.

Before proving Lemmas 3.13 and 3.15, let us look at the entropies of the three examples 2.3,2.7, and 2.8:

3.17. Example. In the $A_{p} B_{1-p}$ and EA models the disorder is confined to the Hamiltonian, and consequently both the sample and skew model entropies are equal to familiar specific relative entropies: Let $\zeta$ be the fair coin-tossing measure of (2.5). Then $s^{\pi}(\mu)=h(\mu \mid \zeta)$ and $\mathbf{s}^{\pi}(Q)=\mathbf{h}(Q \mid \zeta \otimes \pi)$.

3.18. Example. The RFIM offers a chance to illustrate the computation of the generalized derivative and entropy. Suppose $\pi$ is an i.i.d. measure on $\mathbb{R}^{\mathbb{Z}^{d}}$ and its marginal $\pi_{o}$ on $\mathbb{R}$ is symmetric and nondegenerate. Then $\lambda$ is the coin-tossing measure $\zeta$. For $-1 \leqq m \leqq 1$, let $\alpha_{m}:=\frac{1-m}{2} \delta_{-1}+\frac{1+m}{2} \delta_{+1}$ be the unique probability measure on $\mathscr{S}=\{-1,+1\}$ with expected spin $m$, and let $\mu_{m}:=\alpha_{m}^{\otimes \mathbb{Z}^{\mathrm{d}}}$ be the 
corresponding i.i.d. measure on $\Omega$. Then $s^{\pi}\left(\mu_{m}\right)=S^{\pi}\left(\alpha_{m}\right)$, and to compute this we look for the generalized derivative $\psi_{m}$ of $\alpha_{m}$ in the form $\psi_{m}(s)=e^{c s}$ for some number $c$. Define a function $m(c)$ by

$$
m(c):=\int \frac{\lambda_{o}^{h}\left(s e^{c s}\right)}{\lambda_{o}^{h}\left(e^{c s}\right)} \pi_{o}(d h)=\int \frac{e^{c+h}-e^{-c-h}}{e^{c+h}+e^{-c-h}} \pi_{o}(d h) .
$$

The measure $\lambda_{o}^{h}$ was defined in (2.6). $m(\infty)=-m(-\infty)=1$ and $m^{\prime}(c)>0$ for all $c$, hence a well-defined inverse $c=c(m)$ exists for $-1<m<1$. As probability measures on $\mathscr{S}$ are uniquely determined by expectations, $\psi_{m}(s)=e^{c(m) s}$. A final thing to notice is that

$$
m(c)=\frac{\partial}{\partial c} \int \log \left(e^{c+h}+e^{-c-h}\right) \pi_{o}(d h) .
$$

Now we can compute, from (3.14), that

$$
\begin{aligned}
S^{\pi}\left(\alpha_{m}\right) & =m c(m)-\int_{0}^{c} \log \left(e^{c+h}+e^{-c-h}\right) \pi_{o}(d h)+\int \log \left(e^{h}+e^{-h}\right) \pi_{o}(d h) \\
& =m c(m)-\int_{0} m(b) d b \\
& =\int_{0}^{m} c(p) d p .
\end{aligned}
$$

We used the identity $m(0)=0=c(0)$ (from the symmetry of $\pi_{o}$ ) and did an integration by parts for the last equality.

In this example $s^{\pi}\left(\mu_{m}\right)>h\left(\mu_{m} \mid \lambda\right)$ for all $m \neq-1,0,1$, so the new entropy differs from specific relative entropy. (This is easiest to check by first computing $h\left(\mu_{m} \mid \lambda\right)=\frac{1}{2}[(1-m) \log (1-m)+(1+m) \log (1+m)]$ and then Taylor expanding to see that $s^{\pi}\left(\mu_{m}\right)>h\left(\mu_{m} \mid \lambda\right)$ holds for small $m$; it follows for all $m$ from convexity and the inequality $s^{\pi}\left(\mu_{m}\right) \geqq h\left(\mu_{m} \mid \lambda\right)$ that is always valid.)

Proof of Lemma 3.13. Set $v(d s, d x):=\psi(s) / \lambda_{o}^{\mathbf{x}}(\psi) \lambda_{o}(d s, d x)$. The goal is to compare the right-hand side of (3.3) with $H\left(v \mid \lambda_{o}\right)$ which equals the right-hand side of (3.14). We shall begin by proving $\widehat{H}\left(\alpha \mid \lambda_{o}^{*}, \pi\right) \geqq H\left(v \mid \lambda_{o}\right)$. Let $\psi_{b}=\psi \wedge b$ for $b$ large but finite. The hypothesis of the lemma implies that $0<\lambda_{o}^{\mathbf{x}}\left(\psi_{b}\right) \leqq \lambda_{o}^{\mathbf{x}}(\psi)<\infty$ for $\pi$-a.a. $\mathbf{x}$. Let $\log ^{+} x=\log (x \vee 1)$ and $\log ^{-} x=-\log (x \wedge 1)$.

Claim 1. For all $b, \varepsilon>0$, we have $0 \leqq \frac{\psi(s)}{\lambda_{o}^{\mathbf{x}}(\psi)} \log ^{-} \frac{\psi_{b}(s)+\varepsilon}{\lambda_{o}^{\mathbf{x}}\left(\psi_{b}\right)+\varepsilon} \leqq \frac{1}{e}$.

Make these observations:

(i) If $0 \leqq x \leqq y$ and $y>0$, then $\frac{x}{y} \leqq \frac{x+\varepsilon}{y+\varepsilon}$.

(ii) If $\psi(s) \neq \psi_{b}(s)$, then $\psi(s)>\psi_{b}(s)=b \geqq \lambda_{o}^{\mathbf{x}}\left(\psi_{b}\right)$ and hence $\log ^{-} \frac{\psi(s)}{\lambda_{o}^{\mathbf{x}}\left(\psi_{b}\right)}$

$$
=0=\log -\frac{\psi_{b}(s)}{\lambda_{o}^{\mathbf{x}}\left(\psi_{b}\right)} .
$$

(iii) The function $\log ^{-}$is decreasing, hence $\log ^{-} \frac{\psi(s)}{\lambda_{o}^{\mathbf{x}}\left(\psi_{b}\right)} \leqq \log ^{-} \frac{\psi(s)}{\lambda_{o}^{\mathbf{x}}(\psi)}$.

Now the following steps prove Claim 1: 
Disordered Lattice Systems

$$
\begin{aligned}
0 \leqq \frac{\psi(s)}{\lambda_{o}^{\mathbf{x}}(\psi)} \log -\frac{\psi_{b}(s)+\varepsilon}{\lambda_{o}^{\mathbf{x}}\left(\psi_{b}\right)+\varepsilon} & \leqq \frac{\psi(s)}{\lambda_{o}^{\mathbf{x}}(\psi)} \log -\frac{\psi_{b}(s)}{\lambda_{o}^{\mathbf{x}}\left(\psi_{b}\right)}=\frac{\psi(s)}{\lambda_{o}^{\mathbf{x}}(\psi)} \log -\frac{\psi(s)}{\lambda_{o}^{\mathbf{x}}\left(\psi_{b}\right)} \\
& \leqq \frac{\psi(s)}{\lambda_{o}^{\mathbf{x}}(\psi)} \log ^{-} \frac{\psi(s)}{\lambda_{o}^{\mathbf{x}}(\psi)} \leqq \frac{1}{e}
\end{aligned}
$$

Claim 2. $\lim _{b \rightarrow \infty} \inf \lim _{\varepsilon \rightarrow 0} \lambda_{o}\left(\frac{\psi}{\lambda_{o}^{*}(\psi)} \log ^{+} \frac{\psi_{b}+\varepsilon}{\lambda_{o}\left(\psi_{b}\right)+\varepsilon}\right) \geqq \lambda_{o}\left(\frac{\psi}{\lambda_{o}^{\cdot}(\psi)} \log ^{+} \frac{\psi}{\lambda_{o}^{\cdot}(\psi)}\right)$.

Fix $b$. Whenever $\psi_{b}(s) \geqq \lambda_{o}^{\mathbf{x}}\left(\psi_{b}\right)$ and $\varepsilon^{\prime}<\varepsilon$, then $\frac{\psi_{b}(s)+\varepsilon}{\lambda_{o}^{\mathbf{x}}\left(\psi_{b}\right)+\varepsilon} \leqq \frac{\psi_{b}(s)+\varepsilon^{\prime}}{\lambda_{o}^{\mathbf{x}}\left(\psi_{b}\right)+\varepsilon^{\prime}}$.

Thus

$$
\lim _{\varepsilon \rightarrow 0} \lambda_{o}\left(\frac{\psi}{\lambda_{o}^{\cdot}(\psi)} \log ^{+} \frac{\psi_{b}+\varepsilon}{\lambda_{o}\left(\psi_{b}\right)+\varepsilon}\right)=\lambda_{o}\left(\frac{\psi}{\lambda_{o}^{*}(\psi)} \log ^{+} \frac{\psi_{b}}{\lambda_{o}\left(\psi_{b}\right)}\right)
$$

holds by monotone convegence. Claim 2 follows as $b \nearrow \infty$ by Fatou's lemma.

The definition (3.3) remains unchanged if the supremum is taken over bounded Borel functions. Thus for all positive $b$ and $\varepsilon$,

$$
\begin{aligned}
\widehat{H}\left(\alpha \mid \lambda_{o}, \pi\right) & \geqq \alpha\left(\log \left(\psi_{b}+\varepsilon\right)\right)-\int \log \lambda_{o}^{\mathbf{x}}\left(\psi_{b}+\varepsilon\right) \pi(d \mathbf{x}) \\
& =\lambda_{o}\left(\frac{\psi}{\lambda_{o}^{\cdot}(\psi)} \log ^{+} \frac{\psi_{b}+\varepsilon}{\lambda_{o}^{\cdot}\left(\psi_{b}\right)+\varepsilon}\right)-\lambda_{o}\left(\frac{\psi}{\lambda_{o}^{\cdot}(\psi)} \log -\frac{\psi_{b}+\varepsilon}{\lambda_{o}^{\cdot}\left(\psi_{b}\right)+\varepsilon}\right) .
\end{aligned}
$$

Claim 1 implies that the second term above is finite and justifies splitting the integral in the above fashion. Secondly, again by Claim 1, the dominated convergence theorem applies to the second term as we let first $\varepsilon \searrow 0$ and then $b / \infty$. Applying Claim 2 to the first term we get

$$
\begin{aligned}
\widehat{H}\left(\alpha \mid \lambda_{o}^{\cdot}, \pi\right) & \geqq \lambda_{o}\left(\frac{\psi}{\lambda_{o}^{\cdot}(\psi)} \log ^{+} \frac{\psi}{\lambda_{o}^{\cdot}(\psi)}\right)-\lambda_{o}\left(\frac{\psi}{\lambda_{o}^{\cdot(\psi)}} \log { }^{-} \frac{\psi}{\lambda_{o}^{\cdot}(\psi)}\right) \\
& =H\left(v \mid \lambda_{o}\right) .
\end{aligned}
$$

To prove the opposite inequality $\widehat{H}\left(\alpha \mid \lambda_{o}^{\bullet}, \pi\right) \leqq H\left(v \mid \lambda_{o}\right)$, we may assume that

$$
\infty>H\left(v \mid \lambda_{o}\right)=\int \lambda_{o}^{\mathbf{x}}\left(\frac{\psi}{\lambda_{o}^{\mathbf{x}}(\psi)} \log \frac{\psi}{\lambda_{o}^{\mathbf{x}}(\psi)}\right) \pi(d \mathbf{x}),
$$

from which it follows that $\lambda_{o}^{\mathbf{x}}(\psi \log \psi)<\infty \pi$-a.s. Let $f \in C_{b}(\mathscr{S})$ be arbitrary, and write

$$
\alpha(f)-\int \log \lambda_{o}^{\mathbf{x}}\left(e^{f}\right) \pi(d \mathbf{x})=\int\left\{\frac{\lambda_{o}^{\mathbf{x}}(f \psi)}{\lambda_{o}^{\mathbf{x}}(\psi)}-\log \lambda_{o}^{\mathbf{x}}\left(e^{f}\right)\right\} \pi(d \mathbf{x}) .
$$

For almost every $\mathbf{x}$, the integrand equals

$$
\begin{aligned}
\frac{\lambda_{o}^{\mathbf{x}}(f \psi)}{\lambda_{o}^{\mathbf{x}}(\psi)}-\frac{\lambda_{o}^{\mathbf{x}}(\psi \log \psi)}{\lambda_{o}^{\mathbf{x}}(\psi)} & +\frac{\lambda_{o}^{\mathbf{x}}(\psi \log \psi)}{\lambda_{o}^{\mathbf{x}}(\psi)}-\log \lambda_{o}^{\mathbf{x}}\left(e^{f}\right) \\
& =\frac{\lambda_{o}^{\mathbf{x}}(\psi[f-\log \psi])}{\lambda_{o}^{\mathbf{x}}(\psi)}-\log \lambda_{o}^{\mathbf{x}}\left(e^{f}\right)+\frac{\lambda_{o}^{\mathbf{x}}(\psi \log \psi)}{\lambda_{o}^{\mathbf{x}}(\psi)}
\end{aligned}
$$




$$
\begin{aligned}
& \leqq \log \frac{\lambda_{o}^{\mathbf{x}}\left(e^{f}\right)}{\lambda_{o}^{\mathbf{x}}(\psi)}-\log \lambda_{o}^{\mathbf{x}}\left(e^{f}\right)+\frac{\lambda_{o}^{\mathbf{x}}(\psi \log \psi)}{\lambda_{o}^{\mathbf{x}}(\psi)} \\
& =\lambda_{o}^{\mathbf{x}}\left(\frac{\psi}{\lambda_{o}^{\mathbf{x}}(\psi)} \log \frac{\psi}{\lambda_{o}^{\mathbf{x}}(\psi)}\right) .
\end{aligned}
$$

This completes the proof of Lemma 3.13.

Before we turn to the proof of Lemma 3.15, an auxiliary fact about entropyminimizing measures:

3.19. Lemma. Suppose $\mu$ and $v$ are probability measures on some measurable space $X, C$ is a convex set of probability measures, $v \in C$, and $H(v \mid \mu) \leqq H(\rho \mid \mu)$ for all $\rho \in C$. Then $\rho \ll v$ for all $\rho \in C$ such that $H(\rho \mid \mu)<\infty$.

Proof. Write $\phi(x)=x \log x$ for short. Suppose $\rho \in C$ is such that $H(\rho \mid \mu)<\infty$ but $\rho \ll v$ fails. Let $A$ be a measurable set such that $\rho(A)>0=v(A)$. For $0 \leqq t \leqq 1$ set $v_{t}=(1-t) v+t \rho$. In the next calculation, note that $d v / d \mu=0$ on $A$, use the convexity of $\phi$ and rearrange:

$$
\begin{aligned}
H\left(v_{t} \mid \mu\right) & =\int \phi\left((1-t) \frac{d v}{d \mu}+t \frac{d \rho}{d \mu}\right) d \mu \\
& \leqq \int_{A} t \frac{d \rho}{d \mu} \log \left(t \frac{d \rho}{d \mu}\right) d \mu+(1-t) \int_{A^{c}} \phi\left(\frac{d v}{d \mu}\right) d \mu+t \int_{A^{c}} \phi\left(\frac{d \rho}{d \mu}\right) d \mu \\
& =(1-t) H(v \mid \mu)+t[H(\rho \mid \mu)+\rho(A) \log t] .
\end{aligned}
$$

For small enough $t, H(\rho \mid \mu)+\rho(A) \log t<0$ and so $H\left(v_{t} \mid \mu\right)<H(v \mid \mu)$, contradicting the choice of $v$ as $v_{t} \in C$ by convexity.

Proof of Lemma 3.15. In view of Lemma 3.13, we only need to show that $\alpha$ has a generalized derivative $\psi$ whenever $\widehat{H}\left(\alpha \mid \lambda_{o}^{*}, \pi\right)<\infty$. Let $v$ be the unique minimizer in (3.5), which exists by the compactness of the sublevel sets and strict convexity of $H\left(\cdot \mid \lambda_{o}\right)$. Let $\phi=d \alpha / d \lambda_{o}$ which exists by (3.a). The proof of Lemma 3.15 is divided into four steps.

Step 1. $H\left(\alpha \otimes \pi \mid \lambda_{o}\right)<\infty$.

This follows from the hypotheses of the lemma. Let $v(d s, d \mathbf{x})=v^{\mathbf{x}}(d s) \pi(d \mathbf{x})$ be a decomposition of $v$ into conditional distributions on $\mathscr{S}$ given $\mathbf{x}$, so that $\alpha=$ $\int v^{\mathbf{x}} \pi(d \mathbf{x})$. By the conditional entropy formula and convexity,

$$
H\left(\alpha \otimes \pi \mid \lambda_{o}\right)=\int H\left(\alpha \mid \lambda_{o}^{\mathbf{y}}\right) \pi(d \mathbf{y}) \leqq \iint H\left(v^{\mathbf{x}} \mid \lambda_{o}^{\mathbf{y}}\right) \pi(d \mathbf{x}) \pi(d \mathbf{y}) .
$$

Note first that $H\left(v \mid \lambda_{o}\right)<\infty$ implies that $H\left(v^{\mathbf{x}} \mid \lambda_{o}^{\mathbf{x}}\right)<\infty$ for a.e. $\mathbf{x}$, secondly that, by assumption, $\lambda_{o}^{\mathbf{x}} \ll \lambda_{o}^{\mathbf{y}}$ for a.e. $\mathbf{x}$ and $\mathbf{y}$. Thus $v^{\mathbf{x}} \ll \lambda_{o}^{\mathbf{x}} \ll \lambda_{o}^{\mathbf{y}}$ and $\log d \nu^{\mathbf{x}} / d \lambda_{o}^{\mathbf{x}} \in L^{1}\left(v^{\mathbf{x}}\right)$, for a.e. $\mathbf{x}$ and $\mathbf{y}$. And we can write

$$
\begin{aligned}
H\left(v^{\mathbf{x}} \mid \lambda_{o}^{\mathbf{y}}\right) & =v^{\mathbf{x}}\left(\log \frac{d v^{\mathbf{x}}}{d \lambda_{o}^{\mathbf{y}}}\right)=v^{\mathbf{x}}\left(\log \frac{d v^{\mathbf{x}}}{d \lambda_{o}^{\mathbf{x}}}+\log \frac{d \lambda_{o}^{\mathbf{x}}}{d \lambda_{o}^{\mathbf{y}}}\right) \\
& \leqq H\left(v^{\mathbf{x}} \mid \lambda_{o}^{\mathbf{x}}\right)+\left\|\log \frac{d \lambda_{o}^{\mathbf{x}}}{d \lambda_{o}^{\mathbf{y}}}\right\|_{L^{\infty}\left(\lambda_{o}^{\mathbf{x}}\right)} .
\end{aligned}
$$

Integrate against $\pi(d \mathbf{x}) \pi(d \mathbf{y})$ to get 


$$
H\left(\alpha \otimes \pi \mid \lambda_{o}\right) \leqq H\left(v \mid \lambda_{o}\right)+\iint\left\|\log \frac{d \lambda_{o}^{\mathbf{x}}}{d \lambda_{o}^{\mathbf{y}}}\right\|_{L^{\infty}\left(\lambda_{o}^{\mathbf{x}}\right)} \pi(d \mathbf{x}) \pi(d \mathbf{y})
$$

and apply (3.16). Step 1 is completed.

Step 2. $\alpha \otimes \pi \sim v$. Lemma 3.19 and Step 1 imply $\alpha \otimes \pi \ll v$. On the other hand, the hypothesis implies $\lambda_{o}^{\mathbf{x}} \sim \lambda_{o}$ for a.e. $\mathbf{x}$, so integrating $\mathbf{x}$ out gives $\lambda_{o} \sim \lambda_{o} \otimes \pi$. $v$ is supported by the set $\{\phi>0\} \times \mathbf{X}$, and on this set $v \ll \lambda_{o} \sim \lambda_{o} \otimes \pi \sim \alpha \otimes \pi$.

Step 3. There are measurable functions $0<a(s), b(\mathbf{x})<\infty$ and a Borel subset $W$ of $\mathscr{S}$ such that $\frac{d v}{d \lambda_{o}}(s, \mathbf{x})=a(s) b(\mathbf{x}) \mathbf{1}_{W}(s, \mathbf{x})$.

Let $F(s, \mathbf{x})=\log d v / d \lambda_{o}(s, \mathbf{x})$, an element of $L^{1}(v)$. We borrow an argument from Csiszár [Cs]. Let $V$ be the linear subspace of $L^{1}(v)$ of functions of the form $g(s)+h(\mathbf{x})$. Suppose $f \in L^{\infty}(v)$ is such that $v(f G)=0$ for all $G \in V$. In other words,

$$
v(f)=\int f(s, \mathbf{x}) g(s) v(d s, d \mathbf{x})=\int f(s, \mathbf{x}) h(\mathbf{x}) v(d s, d \mathbf{x})=0
$$

for all $g$ and $h$, hence $d v_{\varepsilon}=(1+\varepsilon f) d v$ defines a probability measure $v_{\varepsilon}$ with marginals $\alpha$ and $\pi$, for all $\varepsilon$ small enough. Since $\varepsilon=0$ minimizes $H\left(v_{\varepsilon} \mid \lambda_{o}\right)$, calculus forces $v(f F)=0$. Since $f$ was arbitrary, $F$ must lie in the closure of $V$ in $L^{1}(v)$. It follows that there are measurable functions $g_{n}(s)$ and $h_{n}(\mathbf{x})$ such that $g_{n}(s)+h_{n}(\mathbf{x}) \rightarrow F(s, \mathbf{x}) v$-a.s., hence by Step 2 also $\alpha \otimes \pi$-a.s. By Lemma 2.3 of $[\mathrm{DV}]$, there are finite measurable functions $u(s)$ and $v(\mathbf{x})$ such that $F(s, \mathbf{x})=$ $u(s)+v(\mathbf{x}) \alpha \otimes \pi$-a.s., and thus $v$-a.s. (Lemma 2.3 in [DV] is stated for a measure of the type $\lambda \otimes \lambda$, but the proof is easily seen to work for any product measure $\alpha \otimes \pi$.) Take $a(s)=e^{u(s)}$ and $b(\mathbf{x})=e^{v(\mathbf{x})}$ to have $d v / d \lambda_{o}=a \otimes b v$-a.s., and then let $W=\left\{d v / d \lambda_{o}>0\right\}$.

Step 4. There is a nonnegative measurable function $\psi(s)$ such that $\frac{d v}{d \lambda_{o}}(s, \mathbf{x})=$ $\frac{\psi(s)}{\lambda_{o}^{\mathbf{x}}(\psi)}$

Put $\psi(s)=a(s) \mathbf{1}_{\{\phi>0\}}(s)$. Then for bounded Borel functions $f$ on $\mathscr{S}$,

$$
\begin{aligned}
\int f[\psi \otimes b] d \lambda_{o} & =\int f \mathbf{1}_{\{\phi>0\} \times \mathbf{X}}[a \otimes b] \mathbf{1}_{W} d \lambda_{o}+\int f \mathbf{1}_{\{\phi>0\} \times \mathbf{X}}[a \otimes b] \mathbf{1}_{W^{c}} d \lambda_{o} \\
& =\int f \mathbf{1}_{\{\phi>0\} \times \mathbf{X}} d v+\int f[a \otimes b] \mathbf{1}_{W^{c} \cap\{\phi>0\} \times \mathbf{X}} d \boldsymbol{\lambda}_{o} .
\end{aligned}
$$

Since $v(\{\phi>0\} \times \mathbf{X})=1$, the above line equals $v(f)$ if $\lambda_{o}\left(W^{c} \cap\{\phi>0\} \times \mathbf{X}\right)=$ 0 . But this is equivalent to $\lambda_{o} \otimes \pi\left(W^{c} \cap\{\phi>0\} \times \mathbf{X}\right)=0$, which in turn is equivalent to $\alpha \otimes \pi\left(W^{c}\right)=0$ (see the proof of Step 2), and by Step 2 this follows from $v\left(W^{c}\right)=0$. Thus $d v / d \lambda_{o}=\psi \otimes b$. For Borel subsets $B$ of $\mathbf{X}$,

$$
\pi(B)=v(\mathscr{S} \times B)=\iint_{\mathscr{S} \times B} \psi \otimes b d \lambda_{o}=\int_{B} \lambda_{o}^{\mathbf{x}}(\psi) b(\mathbf{x}) \pi(d \mathbf{x})
$$

hence $b(\mathbf{x})=\lambda_{o}^{\mathbf{x}}(\psi)^{-1}, \pi$-a.s. It is clear that $\psi$ is a generalized derivative of $\alpha$, and so this completes the proof of Lemma 3.15. 


\section{The Finite Volume Model}

Fix $\Lambda \subset \subset \mathbb{Z}^{d}$ and $\pi \in \mathscr{M}_{1}(\mathbf{X})$. For each $\mathbf{x} \in \mathbf{X}$, the equilibrium state $G_{A}^{0, \mathbf{x}}$ on $\Omega_{\Lambda}$ defined by

$$
d G_{\Lambda}^{0, \mathbf{x}}=\frac{e^{-H_{\Lambda}^{0, \mathbf{x}}}}{Z_{\Lambda}^{0, \mathbf{x}}} d \lambda_{\Lambda}^{\mathbf{x}}
$$

is the unique probability measure that satisfies the variational principle [Is, p. 46]:

$$
\begin{aligned}
\inf _{\alpha \in \mathscr{M}_{1}\left(\Omega_{\Lambda}\right)}\left\{\alpha\left(H_{\Lambda}^{0, \mathbf{x}}\right)+H\left(\alpha \mid \lambda_{\Lambda}^{\mathbf{x}}\right)\right\} & =-\log Z_{\Lambda}^{0, \mathbf{x}} \\
& =G_{\Lambda}^{0, \mathbf{x}}\left(H_{\Lambda}^{0, \mathbf{x}}\right)+H\left(G_{\Lambda}^{0, \mathbf{x}} \mid \lambda_{\Lambda}^{\mathbf{x}}\right) .
\end{aligned}
$$

In this section we identify the energy and entropy functions that permit this variational principle to hold for the skew and sample model, while averaging over quenched variables. For probability measures $q$ on $\boldsymbol{\Omega}_{\Lambda}$, the energy in $q$ is defined by

$$
\mathbf{U}(q)=\mathbf{U}_{\Lambda}(q):=q\left(H_{\Lambda}^{0}\right)
$$

and we set

$$
\mathbf{S}(q)=\mathbf{S}_{\Lambda}^{\pi}(q):=\widehat{H}\left(q \mid \dot{\lambda}_{\Lambda}, \pi\right) .
$$

The skew equilibrium state is the probability measure $v$ on $\boldsymbol{\Omega}_{\Lambda}$ defined by

$$
v\left(d \sigma_{\Lambda}, d \mathbf{x}\right)=G_{\Lambda}^{0, \mathbf{x}}\left(d \sigma_{\Lambda}\right) \pi(d \mathbf{x}) .
$$

Let

$$
\wp=\wp_{\Lambda}^{\pi}:=\int \log Z_{\Lambda}^{0, \mathbf{x}} \pi(d \mathbf{x})
$$

be the averaged finite volume pressure.

4.6. Theorem. For all probability measures $q \neq v$ on $\boldsymbol{\Omega}_{\Lambda}$, we have

$$
\mathbf{U}(q)+\mathbf{S}(q)>-\wp=\mathbf{U}(v)+\mathbf{S}(v) .
$$

Proof. By convex duality and (3.3) applied to $\mathbf{S}_{\Lambda}^{\pi}$,

$$
-\wp=\inf _{q \in \mathscr{M}_{1}\left(\boldsymbol{\Omega}_{\Lambda}\right)}\{\mathbf{U}(q)+\mathbf{S}(q)\} .
$$

Integrate (4.1) against $\pi(d \mathbf{x})$ and use (3.4) to get $-\wp=\mathbf{U}(v)+\mathbf{S}(v)$. Let $q$ be an arbitrary measure such that $-\wp=\mathbf{U}(q)+\mathbf{S}(q)$. Then $\mathbf{S}(q)<\infty$, so by (3.4) $q_{\mathbf{x}}=\pi$ and

$$
-\int \log Z_{\Lambda}^{0, \mathbf{x}} \pi(d \mathbf{x})=\int\left[q^{\mathbf{x}}\left(H_{\Lambda}^{0, \mathbf{x}}\right)+H\left(q^{\mathbf{x}} \mid \lambda_{\Lambda}^{\mathbf{x}}\right)\right] \pi(d \mathbf{x}) .
$$

By the first equality of (4.1), the integrands on the left and right must coincide $\pi$-a.s., and so by the uniqueness in (4.1) $q^{\mathbf{x}}=G_{\Lambda}^{0, \mathbf{x}} \pi$-a.s. Hence $q=v$.

Next we find the form that Theorem 4.6 takes in the sample model. For a probability measure $\alpha$ on $\Omega_{\Lambda}$, set

$$
S(\alpha)=S_{\Lambda}^{\pi}(\alpha):=\widehat{H}\left(\alpha \mid \lambda_{\Lambda}, \pi\right) .
$$

For $\alpha$ such that $S(\alpha)<\infty$, define the quantity $U(\alpha)=U_{\Lambda}^{\pi}(\alpha)$ by the equation 
Disordered Lattice Systems

$$
U(\alpha)+S(\alpha)=\inf _{q_{\Omega_{\Lambda}}=\alpha}\{\mathbf{U}(q)+\mathbf{S}(q)\}
$$

Let $\gamma:=v_{\Omega_{\Lambda}}$ be the $\Omega_{\Lambda}$-marginal of $v$. The next statement is immediate from Theorem 4.6.

4.9. Corollary. For all probability measures $\alpha \neq \gamma$ on $\Omega_{\Lambda}$,

$$
U(\alpha)+S(\alpha)>-\wp=U(\gamma)+S(\gamma) .
$$

At this point the quantity $U(\alpha)$ is purely an ad hoc construction designed to reproduce the finite volume variational principle in the sample model. It gains some mathematical legitimacy once we see in Sect. 5 and 8 that it has a well-defined infinite volume limit that occupies the natural role in the infinite volume variational principle. From (3.5) it is immediate that $U(\alpha)=\alpha\left(H_{\Lambda}^{0}\right)$ whenever the Hamiltonian $H_{\Lambda}^{0, \mathbf{x}}$ is independent of the quenched variable $\mathbf{x}$. Thus $U$ is a generalization of the energy function of statistical mechanics without disorder.

$U(\alpha)$ has an alternative expression as the difference of two convex duals of pressure-type functionals. For bounded continuous functions $f$ on $\Omega_{\Lambda}$, set

$$
P(f):=\int \log \lambda_{\Lambda}^{\mathbf{x}}\left(e^{f-H_{\Lambda}^{0, \mathbf{x}}}\right) \pi(d \mathbf{x}),
$$

its convex dual on measures defined by

$$
P^{*}(\alpha):=\sup _{f \in C_{b}\left(\Omega_{\Lambda}\right)}\{\alpha(f)-P(f)\} .
$$

4.10. Lemma. $U(\alpha)=P^{*}(\alpha)-S(\alpha)$ whenever $S(\alpha)<\infty$.

Proof. Extend $P$ to functions $g \in C_{b}\left(\boldsymbol{\Omega}_{\Lambda}\right)$ by defining

$$
\Pi(g):=\int \log \lambda_{\Lambda}^{\mathbf{x}}\left(e^{g-H_{\Lambda}^{0}}\right) \pi(d \mathbf{x}) .
$$

The arguments used in the proof of Lemma 5.25 below can be used to see that $\Pi$ is weakly lower semicontinuous and convex, $\Pi=\Pi^{* *}$, and that, for $\eta \in C_{b}\left(\boldsymbol{\Omega}_{\Lambda}\right)^{*}, \Pi^{*}(\eta)<\infty$ only if $\eta$ is given by a probability measure on $\boldsymbol{\Omega}_{\Lambda}$ with $\mathbf{X}$-marginal $\pi$. Let

$$
F(\alpha):=\inf _{q_{\Omega_{\Lambda}}=\alpha} \Pi^{*}(q)
$$

for measures $\alpha$ on $\Omega_{\Lambda}$; it is finite only for probability measures. From

$$
q(g) \leqq \log \lambda_{\Lambda}\left(e^{g}\right)+\left\|H_{\Lambda}^{0}\right\|+\Pi^{*}(q),
$$

valid for all $g$, we see that the sets $\left\{\Pi^{*} \leqq c\right\}$ are compact. Thus $F$ is convex and lower semicontinuous and consequently $F=F^{* *}$. For $f \in C_{b}\left(\Omega_{\Lambda}\right)$,

$$
\begin{aligned}
P(f) & =\Pi(f)=\Pi^{* *}(f)=\sup _{q \in \mathscr{M}_{1}\left(\mathbf{\Omega}_{\Lambda}\right)}\left\{q_{\Omega_{\Lambda}}(f)-\Pi^{*}(q)\right\} \\
& =\sup _{\alpha \in \mathscr{M}_{1}\left(\boldsymbol{\Omega}_{\Lambda}\right)}\{\alpha(f)-F(\alpha)\} \\
& =F^{*}(f) .
\end{aligned}
$$

Then, for $\alpha \in \mathscr{M}_{1}\left(\Omega_{\Lambda}\right)$, 


$$
\begin{aligned}
P^{*}(\alpha) & =F^{* *}(\alpha)=F(\alpha)=\inf _{q_{\Omega_{\Lambda}}=\alpha} \sup _{g \in C_{b}\left(\boldsymbol{\Omega}_{\Lambda}\right)}\{q(g)-\Pi(g)\} \\
& =\inf _{q_{\Omega_{\Lambda}}=\alpha} \sup _{g \in C_{b}\left(\boldsymbol{\Omega}_{\Lambda}\right)}\left\{q(g)-\int \log \lambda_{\Lambda}^{\mathbf{x}}\left(e^{g}\right) \pi(d \mathbf{x})+q\left(H_{\Lambda}^{0}\right)\right\} \\
& =\inf _{q_{\Omega_{\Lambda}}=\alpha}\{\mathbf{S}(q)+\mathbf{U}(q)\} \\
& =U(\alpha)+S(\alpha) .
\end{aligned}
$$

The measures $v$ and $\gamma$ also minimize entropy under an energy constraint. For the skew model it is clear from Theorem 4.6 that if $\mathbf{U}(v)=c$, then $q=v$ is the unique minimizer of $\mathbf{S}(q)$ subject to $\mathbf{U}(q)=c$. The same holds also for the sample model:

4.11. Proposition. Let $c$ be real and suppose that $U(\gamma)=c$. Then $\alpha=\gamma$ is the unique minimizer of $S(\alpha)$ subject to $U(\alpha)=c$.

Proof. Suppose that $U(\alpha)=c$ and $S(\alpha) \leqq S(\gamma)$ for some $\alpha \in \mathscr{M}_{1}\left(\Omega_{\Lambda}\right)$. By the lower semicontinuity and compact level sets of the function $\mathbf{U}+\mathbf{S}$ on $\mathscr{M}_{1}\left(\boldsymbol{\Omega}_{\Lambda}\right)$, there is a $q$ with $q_{\Omega_{\Lambda}}=\alpha$ that realizes the infimum on the right-hand side of (4.8). Then

$$
\mathbf{U}(q)+\mathbf{S}(q)=c+S(\alpha) \leqq c+S(\gamma)=\mathbf{U}(v)+\mathbf{S}(v) .
$$

By Theorem $4.6 q=v$, and consequently $\alpha=\gamma$.

Let us compare $v$ and $\gamma$ with the equilibrium state $\mu$ under annealed disorder where the quenched variable $\mathbf{x}$ participates in the thermal equilibrium. The a priori measure is now $\lambda_{\Lambda}:=\int \lambda_{\Lambda}^{\mathbf{x}} \pi(d \mathbf{x})$, the partition function is

$$
Z_{\Lambda}^{0}:=\int_{\mathbf{\Omega}_{\Lambda}} e^{-H_{\Lambda}^{0}} d \lambda_{\Lambda}=\int_{\mathbf{X}} Z_{\Lambda}^{0, \mathbf{x}} \pi(d \mathbf{x})
$$

and the equilibrium measure on $\boldsymbol{\Omega}_{\Lambda}$, as given by the usual Gibbs prescription, is

$$
d \mu:=\frac{1}{Z_{\Lambda}^{0}} e^{-H_{\Lambda}^{0}} d \lambda_{\Lambda} .
$$

To read off the effect of annealed disorder, let us rewrite this as

$$
\mu\left(d \sigma_{\Lambda}, d \mathbf{x}\right)=\frac{e^{-H_{\Lambda}^{0, \mathbf{x}}\left(\sigma_{\Lambda}\right)}}{Z_{\Lambda}^{0, \mathbf{x}}} \lambda_{\Lambda}^{\mathbf{x}}\left(d \sigma_{\Lambda}\right) \frac{Z_{\Lambda}^{0, \mathbf{x}}}{Z_{\Lambda}^{0}} \pi(d \mathbf{x})=G_{\Lambda}^{0, \mathbf{x}}\left(d \sigma_{\Lambda}\right) \frac{Z_{\Lambda}^{0, \mathbf{x}}}{Z_{\Lambda}^{0}} \pi(d \mathbf{x}) .
$$

Thus when we restrict to that part of the space $\boldsymbol{\Omega}_{\Lambda}$ where $\mathbf{x}$ is fixed, the equilibrium of the spins $\sigma_{\Lambda}$ under $\mu$ is still given by $G_{\Lambda}^{0, \mathbf{x}}$. Mathematically speaking, $G_{\Lambda}^{0, \mathbf{x}}$ is the conditional distribution of $\mu$ on $\Omega_{\Lambda}$, given $\mathbf{x}$. But the probabilities of quenched variables change from the a priori distribution $\pi(d \mathbf{x})$ to the equilibrium distribution $Z_{\Lambda}^{0, \mathbf{x}} / Z_{\Lambda}^{0} \pi(d \mathbf{x})$. In the annealed equilibrium the spins do not obey the sample equilibrium state $\gamma=\int G_{\Lambda}^{0, \mathbf{x}} \pi(d \mathbf{x})$ but another mixture of the kernels $G_{\Lambda}^{0, \mathbf{x}}$, namely

$$
\mu_{\Omega_{\Lambda}}=\int G_{\Lambda}^{0, \mathbf{x}} \frac{Z_{\Lambda}^{0, \mathbf{x}}}{Z_{\Lambda}^{0}} \pi(d \mathbf{x})
$$




\section{Thermodynamic Limits}

Let $\pi \in \mathscr{M}_{\Theta}(\mathbf{X})$. From the development in Sect. 3 we have the infinite volume entropies $s^{\pi}(\mu)=\widehat{h}\left(\mu \mid \lambda^{*}, \pi\right)$ for the sample model and $\mathbf{s}^{\pi}(Q)=\widehat{h}\left(Q \mid \hat{\lambda}^{*}, \pi\right)$ for the skew model that satisfy

$$
s^{\pi}(\mu)=\lim _{n \rightarrow \infty} \frac{1}{\left|V_{n}\right|} S_{n}^{\pi}\left(\mu_{n}\right)=\sup _{\Lambda \in \mathscr{R}} \frac{1}{|\Lambda|} S_{\Lambda}^{\pi}\left(\mu_{\Lambda}\right)
$$

and

$$
\mathbf{s}^{\pi}(Q)=\lim _{n \rightarrow \infty} \frac{1}{\left|V_{n}\right|} \mathbf{S}_{n}^{\pi}\left(Q_{n}\right)=\sup _{\Lambda \in \mathscr{R}} \frac{1}{|\Lambda|} \mathbf{S}_{\Lambda}^{\pi}\left(Q_{\Lambda}\right)
$$

for all invariant measures $\mu \in \mathscr{M}_{\Theta}(\Omega)$ and $Q \in \mathscr{M}_{\Theta}(\boldsymbol{\Omega})$. This section presents the infinite volume limits of the other two central quantities, namely energy and pressure. For the energy of the skew model this involves nothing but invariance:

5.3. Lemma. Let $Q \in \mathscr{M}_{\Theta}(\boldsymbol{\Omega})$. Then

$$
\mathbf{u}(Q):=\lim _{n \rightarrow \infty} \frac{1}{\left|V_{n}\right|} \mathbf{U}_{n}\left(Q_{n}\right)
$$

exists and satisfies $\mathbf{u}(Q)=Q(\varphi)$. In particular, $\mathbf{u}(Q)$ is a continuous function of $Q$.

The "energy" $U_{1}^{\pi}$ of the sample model was defined somewhat indirectly in terms of convex duals, hence it is not as evident that it should behave well under the thermodynamic limit. We have the following result:

5.4. Proposition. Let $\pi \in \mathscr{M}_{\Theta}(\mathbf{X})$. For $\mu \in \mathscr{M}_{\Theta}(\Omega)$ such that $s^{\pi}(\mu)<\infty$, the thermodynamic limit

$$
u^{\pi}(\mu)=\lim _{n \rightarrow \infty} \frac{1}{\left|V_{n}\right|} U_{n}^{\pi}\left(\mu_{n}\right)
$$

exists and satisfies

$$
u^{\pi}(\mu)+s^{\pi}(\mu)=\inf _{Q_{\Omega}=\mu}\left\{\mathbf{u}(Q)+\mathbf{s}^{\pi}(Q)\right\} .
$$

This defines a Borel function $u^{\pi}$ on the nonempty convex subset $\left\{s^{\pi}<\infty\right\}$ of $\mathscr{M}_{\Theta}(\Omega)$, with $\left|u^{\pi}(\mu)\right| \leqq\|\varphi\|$. If $\pi$ is ergodic, then $u^{\pi}$ is affine.

The thermodynamic limit of the pressure, as a nonrandom a.s. limit typically under i.i.d. quenched variables, has been proved many times over for disordered models. Our point of view is a little different: We do not work with a fixed distribution on the quenched variables, but we show that the infinite volume pressure $\wp^{\mathbf{x}}$ is a well-defined function on the set of all generic quenched variables, and that $\wp^{\mathbf{x}}$ is naturally related to an infinite volume pressure $\wp^{\pi}$ defined as a function on invariant distributions $\pi$.

\subsection{Theorem.}

(i) For each generic variable $\mathbf{x}$ there is an infinite volume pressure $\wp^{\mathbf{x}}$ such that $\mathbf{x} \mapsto \wp^{\mathbf{x}}$ is a bounded Borel function and

$$
\lim _{n \rightarrow \infty} \sup _{\sigma \in \Omega}\left|\wp^{\mathbf{x}}-\frac{1}{\left|V_{n}\right|} \log Z_{n}^{\sigma, \mathbf{x}}\right|=0 \text {. }
$$


(ii) For each invariant probability distribution $\pi$ on $\mathbf{X}$ there is an infinite volume pressure $\wp^{\pi}$ such that $\pi \mapsto \wp^{\pi}$ is an upper semicontinuous bounded affine function on $\mathscr{M}_{\Theta}(\mathbf{X})$, and

$$
\lim _{n \rightarrow \infty} \sup _{\sigma \in \Omega}\left|\wp^{\pi}-\frac{1}{\left|V_{n}\right|} \int \log Z_{n}^{\sigma, \mathbf{x}} \pi(d \mathbf{x})\right|=0
$$

(iii) The functions $\wp^{\mathbf{x}}$ and $\wp^{\pi}$ are connected by

$$
\wp^{\pi}=\int \wp^{\mathbf{x}} \pi(d \mathbf{x})
$$

valid for all $\pi \in \mathscr{M}_{\Theta}(\mathbf{X})$, and $\wp^{\mathbf{x}}=\wp^{\pi}$ whenever $\mathbf{x}$ is generic for $\pi$. In variational terms,

$$
-\wp^{\pi}=\inf _{Q \in \mathscr{M}_{\Theta}(\boldsymbol{\Omega})}\left\{\mathbf{u}(Q)+\mathbf{s}^{\pi}(Q)\right\}=\inf _{\mu \in \mathscr{M}_{\Theta}(\Omega)}\left\{u^{\pi}(\mu)+s^{\pi}(\mu)\right\}
$$

Quenched randomness refers to the situation where thermal averages are taken under a fixed quenched variable $\mathbf{x}$. From this perspective, the interesting measures on $\mathbf{X}$ are those that can be realized as a limit of averages $\left|V_{n}\right|^{-1} \sum_{i \in V_{n}} \delta_{\theta_{i} \mathbf{x}}$ for some $\mathbf{x}$, in other words those that have generic quenched variables. As remarked earlier, all ergodic measures belong to this class, but there can be other measures too, depending on $\mathbf{X}$ and the $\mathbb{Z}^{d}$ action on it. If $\mathbf{X}$ is a configuration space $\mathbf{X}=E^{\mathbb{Z}^{d}}$ and $\mathbb{Z}^{d}$ acts by shifts, then every invariant measure on $\mathbf{X}$ has a generic quenched variable by Lemma 3.1 in [Se1].

Suppose $\pi \in \mathscr{M}_{\Theta}(\mathbf{X})$ has a generic quenched variable $\mathbf{x}$ but is not ergodic, and $\pi=\int v w(d v)$ is its ergodic decomposition. Theorem 5.6 tells us that

$$
\int \wp^{v} w(d v)=\wp^{\pi}=\wp^{\mathbf{x}}=\lim _{n \rightarrow \infty} \frac{1}{\left|V_{n}\right|} \log Z_{n}^{0, \mathbf{x}} .
$$

The interesting point is that the quenched variables generic for nonergodic measures are atypical in the sense that

$$
v\{\mathbf{x}: \mathbf{x} \text { is generic for a nonergodic measure }\}=0
$$

for any $v \in \mathscr{M}_{\Theta}(\mathbf{X})$, but nevertheless such a quenched variable yields the correct limit in (5.11).

Write $\wp^{\pi}(\boldsymbol{\Phi})$ to indicate the dependence of the pressure on the interaction $\boldsymbol{\Phi}$. The familiar result about pressure-bounded linear functionals on interactions [Is, Theorem II.1.2] now takes the following form:

5.12. Proposition. Let $\pi \in \mathscr{M}_{\Theta}(\mathbf{X})$ and $\xi \in \mathbb{B}^{*}$. Suppose there exists a constant C such that

$$
\xi(\boldsymbol{\Phi}) \leqq \wp^{\pi}(\boldsymbol{\Phi})+C
$$

for all $\boldsymbol{\Phi} \in \mathbb{B}$. Then there is a unique $Q \in \mathscr{M}_{\boldsymbol{\Theta}}^{\pi}(\boldsymbol{\Omega})$ such that $\xi(\boldsymbol{\Phi})=-Q(\varphi)$ for all $\boldsymbol{\Phi} \in \mathbb{B}$, where $\varphi$ is associated to $\boldsymbol{\Phi}$ as in (2.2).

The rest of this section is devoted to the proofs. For readers familiar with the use of large deviation theory it perhaps suffices to say that Theorem 5.6 follows from the large deviation principle stated in (3.e) and some convex analysis. Proposition 5.12 is proved as in [Is] with a few additional technicalities. 
Proof of Lemma 5.3. Note first that for any $\Delta \subset \Lambda \subset \subset \mathbb{Z}^{d}$,

$$
\left\|H_{\Lambda}^{0}-H_{\Lambda}\right\| \leqq c_{\Delta, \Lambda}
$$

and

$$
\left\|H_{\Lambda}^{0}-\sum_{\mathbf{i} \in \Lambda} \varphi \circ \theta_{\mathbf{i}}\right\| \leqq 2 c_{\Delta, \Lambda}
$$

with

$$
c_{\Delta, \Lambda}:=|\Lambda| \cdot \sum_{\substack{A \ni \mathbf{0} \\ A \not \subset \Delta}}\left\|\Phi_{A}\right\|+\|\boldsymbol{\Phi}|\| \cdot|\{\mathbf{i} \in \Lambda: \mathbf{i}+\Delta \not \subset \Lambda\} \mid .
$$

By $Q$ 's invariance, (4.2), and (5.15),

$$
Q(\varphi)=Q\left(\mathbf{R}_{n} \varphi\right)=\frac{1}{\left|V_{n}\right|} \mathbf{U}_{n}\left(Q_{n}\right)+O\left(\frac{c_{r, n}}{\left|V_{n}\right|}\right)
$$

for any $r<n$. Now let first $n \rightarrow \infty$ and then $r \rightarrow \infty$ and note that

$$
\lim _{r \rightarrow \infty} \lim _{n \rightarrow \infty} \frac{c_{r, n}}{\left|V_{n}\right|}=0
$$

Proof of Proposition 5.4. For $Q \in \mathscr{M}_{\Theta}(\boldsymbol{\Omega})$, set

$$
\mathbf{f}(Q):=\mathbf{u}(Q)+\mathbf{s}^{\pi}(Q)=\lim _{n \rightarrow \infty} \frac{1}{\left|V_{n}\right|}\left[\mathbf{U}_{n}\left(Q_{n}\right)+\mathbf{S}_{n}^{\pi}\left(Q_{n}\right)\right] .
$$

For $\alpha \in \mathscr{M}_{1}\left(\Omega_{n}\right)$ and $\mu \in \mathscr{M}_{\Theta}(\Omega)$ such that $S_{n}^{\pi}(\alpha)<\infty$ and $s^{\pi}(\mu)<\infty$, set

$$
\begin{gathered}
F_{n}(\alpha):=U_{n}^{\pi}(\alpha)+S_{n}^{\pi}(\alpha)=\inf _{q_{\Omega_{n}}=\alpha}\left\{\mathbf{U}_{n}(q)+\mathbf{S}_{n}^{\pi}(q)\right\}, \\
f(\mu):=\inf _{Q_{\Omega}=\mu} \mathbf{f}(Q), \\
\bar{f}(\mu):=\lim _{n \rightarrow \infty} \sup _{\frac{1}{\left|V_{n}\right|} F_{n}\left(\mu_{n}\right),} \text { and } \underline{f}(\mu):=\liminf _{n \rightarrow \infty} \frac{1}{\left|V_{n}\right|} F_{n}\left(\mu_{n}\right) .
\end{gathered}
$$

Note that $s^{\pi}(\mu)<\infty$ and (3.11) together imply that $|f(\mu)|<\infty$.

Let us first show that

$$
f(\mu)=\lim _{n \rightarrow \infty} \frac{1}{\left|V_{n}\right|} F_{n}\left(\mu_{n}\right),
$$

by deducing $\bar{f}(\mu) \leqq f(\mu) \leqq f(\mu)$. By the compact sublevel sets and lower semicontinuity of $\mathbf{u}+\mathbf{s}^{\pi}$, there exists a $Q \in \mathscr{M}_{\Theta}(\boldsymbol{\Omega})$ with $Q_{\Omega}=\mu$ and $f(\mu)=\mathbf{f}(Q)$. Then

$$
f(\mu)=\lim _{n \rightarrow \infty} \frac{1}{\left|V_{n}\right|}\left[\mathbf{U}_{n}\left(Q_{n}\right)+\mathbf{S}_{n}^{\pi}\left(Q_{n}\right)\right] \geqq \limsup _{n \rightarrow \infty} \frac{1}{\left|V_{n}\right|} F_{n}\left(\mu_{n}\right)=\bar{f}(\mu) .
$$

Next, fix $n$ and let $q \in \mathscr{M}_{1}\left(\boldsymbol{\Omega}_{n}\right)$ be such that $q_{\Omega_{n}}=\mu_{n}$ and

$$
F_{n}\left(\mu_{n}\right)=\mathbf{U}_{n}(q)+\mathbf{S}_{n}^{\pi}(q) .
$$

Let $q^{\mathbf{x}}$ be a version of the conditional distribution of $q$ on $\Omega_{n}$, given $\mathbf{x} . H:=(2 n+$ $1) \mathbb{Z}^{d}$ is the subgroup of $\mathbb{Z}^{d}$ that makes $\left\{\mathbf{j}+V_{n}: \mathbf{j} \in H\right\}$ into a disjoint covering 
of $\mathbb{Z}^{d}$. The following construction extends $q$ to a probability measure $q^{\otimes}$ on $\boldsymbol{\Omega}$ such that the variables $\left\{\left(\sigma_{\mathbf{j}+V_{n}}, \mathbf{x}\right): \mathbf{j} \in H\right\}$, have identical distribution $q$ and are independent, given $\mathbf{x}$ :

$$
q^{\otimes}(d \sigma, d \mathbf{x}):=\left[\underset{\mathbf{j} \in H}{\otimes} q^{\theta_{\mathbf{j}} \mathbf{x}}\left(d \sigma_{\mathbf{j}+V_{n}}\right)\right] \pi(d \mathbf{x}) .
$$

$Q^{n}:=\left|V_{n}\right|^{-1} \sum_{\mathbf{i} \in V_{n}} q^{\otimes} \circ \theta_{-\mathbf{i}}$ defines an invariant probability measure. By (5.15),

$$
\mathbf{u}\left(Q^{n}\right)=\frac{1}{\left|V_{n}\right|} \mathbf{U}_{n}(q)+O\left(\frac{c_{r, n}}{\left|V_{n}\right|}\right) .
$$

For $k$ odd, choose $m_{k}$ so that $H_{k}:=\left\{\mathbf{j} \in H:-m_{k}<\mathbf{j}_{1}, \ldots, \mathbf{j}_{d}<m_{k}\right\}$ has cardinality $\left|H_{k}\right|=k^{d}$ and $V_{m_{k}}=\bigcup_{\mathbf{j} \in H_{k}}\left(\mathbf{j}+V_{n}\right)$. For each $\mathbf{i} \in V_{n}$, let $W_{\mathbf{i}}$ be the union of those sets $\mathbf{i}+\mathbf{j}+V_{n}, \mathbf{j} \in H$, that intersect $V_{m_{k}}$. Then $W_{\mathbf{i}}$ is a union of at most $(k+1)^{d}$ disjoint shifted copies of $V_{n}$. By (3.4) and the independence built into $q^{\otimes}$,

$$
\mathbf{S}_{W_{\mathbf{i}}}^{\pi}\left(\left(q^{\otimes} \circ \theta_{-\mathbf{i}}\right)_{W_{\mathbf{i}}}\right) \leqq(k+1)^{d} \mathbf{S}_{n}^{\pi}(q)
$$

Since $\mathbf{S}_{\Lambda}^{\pi}(\rho)$ is convex in $\rho$ and increasing in $\Lambda$,

$$
\begin{aligned}
\mathbf{S}_{m_{k}}^{\pi}\left(Q_{m_{k}}^{n}\right) & \leqq \frac{1}{\left|V_{n}\right|} \sum_{\mathbf{i} \in V_{n}} \mathbf{S}_{m_{k}}^{\pi}\left(\left(q^{\otimes} \circ \theta_{-\mathbf{i}}\right)_{m_{k}}\right) \\
& \leqq \frac{1}{\left|V_{n}\right|} \sum_{\mathbf{i} \in V_{n}} \mathbf{S}_{W_{\mathbf{i}}}^{\pi}\left(\left(q^{\otimes} \circ \theta_{-\mathbf{i}}\right)_{W_{\mathbf{i}}}\right) \\
& \leqq(k+1)^{d} \mathbf{S}_{n}^{\pi}(q)
\end{aligned}
$$

Divide by $\left|V_{m_{k}}\right|$ and let $k \rightarrow \infty$ to get

$$
\mathbf{s}^{\pi}\left(Q^{n}\right) \leqq \frac{1}{\left|V_{n}\right|} \mathbf{S}_{n}^{\pi}(q)
$$

Equations (5.20)-(5.22) combine to yield

$$
\frac{1}{\left|V_{n}\right|} F_{n}\left(\mu_{n}\right) \geqq \mathbf{u}\left(Q^{n}\right)+\mathbf{s}^{\pi}\left(Q^{n}\right)+O\left(\frac{c_{r, n}}{\left|V_{n}\right|}\right) .
$$

Repeat this for all $n$. The sequence $\left\{Q^{n}\right\}$ is tight, for its $\Omega$-marginals converge to $\mu$ and its $\mathbf{X}$-marginals are constantly $\pi$. Hence $\left\{Q^{n}\right\}$ has limit points, and any limit point $Q$ satisfies $Q \in \mathscr{M}_{\Theta}(\boldsymbol{\Omega})$ and $Q_{\Omega}=\mu$. Thus we may let first $n \rightarrow \infty$ in (5.23) along a suitable subsequence, use the continuity of $\mathbf{u}$ and the lower semicontinuity of $\mathbf{s}^{\pi}$, and then let $r \rightarrow \infty$ to get $\underline{f}(\mu) \geqq \mathbf{u}(Q)+\mathbf{s}^{\pi}(Q) \geqq f(\mu)$. This completes the proof of (5.19).

Set

$$
u^{\pi}(\mu):=f(\mu)-s^{\pi}(\mu)=\lim _{n \rightarrow \infty} \frac{1}{\left|V_{n}\right|}\left[F_{n}\left(\mu_{n}\right)-S_{n}^{\pi}\left(\mu_{n}\right)\right]=\lim _{n \rightarrow \infty} \frac{1}{\left|V_{n}\right|} U_{n}^{\pi}\left(\mu_{n}\right) .
$$

Equation (5.5) is the same as (5.18). The inequality $\left|u^{\pi}(\mu)\right| \leqq\|\varphi\|$ follows from (5.5). 
Assume $\pi$ is ergodic. Then $s^{\pi}$ is affine by (3.d), so to prove $u^{\pi}$ affine it suffices to show that $f$ is affine. $\mathbf{f}$ is affine, lower semicontinuous, and has compact sublevel sets by (3.e) and Lemma 5.3, hence $f$ is convex and lower semicontinuous by (5.18). To show $f$ is concave, it suffices to show that

$$
f(\mu) \geqq \int f(\rho) w(d \rho)
$$

whenever $\mu=\int \rho w(d \rho)$ is the ergodic decomposition of $\mu \in \mathscr{M}_{\Theta}(\Omega)$. Choose $Q \in \mathscr{M}_{\boldsymbol{\Theta}}^{\pi}(\boldsymbol{\Omega})$ such that $Q_{\Omega}=\mu$ and $f(\mu)=\mathbf{f}(Q)$, with ergodic decomposition $Q=\int R \mathbf{w}(d R)$. Since $Q_{\mathbf{X}}=\pi$ is ergodic, $R_{\mathbf{X}}=\pi \mathbf{w}$-a.s. Lemma 5.4.24 in [DS] applied to $\mathbf{f},(5.18)$, and the uniqueness of $w$ in the ergodic decomposition give

$$
f(\mu)=\mathbf{f}(Q)=\int \mathbf{f}(R) \mathbf{w}(d R) \geqq \int f\left(R_{\Omega}\right) \mathbf{w}(d R)=\int f(\rho) w(d \rho) .
$$

For $\mathbf{x} \in \mathbf{X}$ and $f \in \overline{\mathscr{C}}$, set

$$
p_{n}(\mathbf{x}, f):=\frac{1}{\left|V_{n}\right|} \log \lambda^{\mathbf{x}}\left(\exp \left\{\left|V_{n}\right| \mathbf{R}_{n}(f)\right\}\right) .
$$

By (3.e) and Varadhan's Theorem [DS, 2.1.10], $p(\mathbf{x}, f):=\lim _{n \rightarrow \infty} p_{n}(\mathbf{x}, f)$ exists and is given by

$$
p(\mathbf{x}, f)=\sup _{Q \in \|_{\Theta}(\boldsymbol{\Omega})}\left\{Q(f)-\mathbf{s}^{\pi}(Q)\right\}
$$

whenever $\mathbf{x}$ is generic for $\pi$. Every $\pi \in \mathscr{M}_{\Theta}(\mathbf{X})$ is supported by generic quenched variables, so it makes sense to define

$$
p(\pi, f):=\int p(\mathbf{x}, f) \pi(d \mathbf{x}) .
$$

For elements $\eta$ of the dual space $\overline{\mathscr{C}}^{*}$ of the Banach space $\overline{\mathscr{C}}$ we define the convex dual of $p(\pi, \cdot)$ by

$$
p^{*}(\pi, \eta):=\sup _{f \in \overline{\mathscr{C}}}\{\eta(f)-p(\pi, f)\} .
$$

5.25. Lemma. Let $\pi \in \mathscr{M}_{\Theta}(\mathbf{X})$.

(a) For $\eta \in \overline{\mathscr{C}}^{*}, p^{*}(\pi, \eta)= \begin{cases}\mathbf{s}^{\pi}(Q) & \text { if } \eta \text { is given by } Q \in \mathscr{M}_{\boldsymbol{\Theta}}(\boldsymbol{\Omega}), \\ \infty & \text { otherwise. }\end{cases}$

(b) For $f \in \overline{\mathscr{C}}, p(\pi, f)=\sup _{Q \in \|_{\Theta}(\Omega)}\left\{Q(f)-\mathbf{s}^{\pi}(Q)\right\}$.

(c) $p(\pi, f)=p(\mathbf{x}, f)$ for $f \in \overline{\mathscr{C}}$ whenever $\mathbf{x}$ is generic for $\pi$.

Proof. (a) Fix $\eta \in \overline{\mathscr{C}}^{*}$ and suppose that $c:=p^{*}(\pi, \eta)<\infty$. We wish to show that, for some $Q \in \mathscr{M}_{\Theta}(\boldsymbol{\Omega}), \eta(f)=Q(f)$ for all $f \in \overline{\mathscr{C}}$.

By the argument of Lemma 5.10 in [Se1],

$$
p(\pi, f) \leqq \frac{1}{\left|V_{k}\right|} \int \log \lambda^{\mathbf{x}}\left(e^{\left|V_{k}\right| f}\right) \pi(d \mathbf{x})
$$

for $f \in \mathscr{C}_{k}$, and an application of Jensen's inequality gives

$$
p(\pi, f) \leqq \frac{1}{\left|V_{k}\right|} \log \lambda\left(e^{\left|V_{k}\right| f}\right) .
$$

Hence we deduce 


$$
\eta(f) \leqq p(\pi, f)+c \leqq \frac{1}{\left|V_{k}\right|} \log \lambda\left(e^{\left|V_{k}\right| f}\right)+c
$$

for all $f \in \mathscr{C}_{k}$. Applying this to both $M \cdot \mathbf{1}_{\boldsymbol{\Omega}}$ and $-M \cdot \mathbf{1}_{\boldsymbol{\Omega}}$ and letting $M \nearrow \infty$ gives $\eta\left(\mathbf{1}_{\boldsymbol{\Omega}}\right)=1$. Next, apply (5.28) to $-M \cdot f$ for $f \geqq 0$ and $M>0$, and note that $p(\pi,-M \cdot f) \leqq 0$ to get $\eta(f) \geqq 0$. Finally, suppose $f_{n} \searrow 0$ pointwise in $\mathscr{C}_{k}$. Apply (5.28) to $M \cdot f_{n}$, use the bounded convergence theorem to let $n \rightarrow \infty$ on the right-hand side, and deduce $\limsup _{n \rightarrow \infty} M \eta\left(f_{n}\right) \leqq c$. This implies $\eta\left(f_{n}\right) \searrow 0$ as $n \rightarrow \infty$.

To summarize, we have shown that $\eta$ is a pre-integral on $\mathscr{C}_{k}$. By the DaniellStone theorem [Du, 4.5.2] and by $\eta\left(\mathbf{1}_{\boldsymbol{\Omega}}\right)=1$ there is a probability measure $Q_{k}$ on $\mathscr{F}_{k}$ such that $\eta(f)=Q_{k}(f)$ for all $f \in \mathscr{C}_{k}$. The measures $Q_{k}$ are consistent, so by Kolmogorov's extension theorem they are the $\widetilde{F}_{k}$-marginals of a probability measure $Q \in \mathscr{M}_{1}(\boldsymbol{\Omega})$. Since $\eta(f)=Q(f)$ for all $f \in \mathscr{C}$, we get $\eta(f)=Q(f)$ for all $f \in \overline{\mathscr{C}}$ by passing to uniform limits. $Q$ has to be invariant by (5.28) because $p\left(\pi, f-f \circ \theta_{\mathbf{i}}\right)=0$ for all $f$ and $\mathbf{i}$.

It remains to show $p^{*}(\pi, \eta)=\mathbf{s}^{\pi}(Q)$. Let $f \in \mathscr{C}_{k}$. Then $\left|V_{n}\right| \mathbf{R}_{n}(f) \in \mathscr{C}_{n+k}$, so by (3.3) and $Q$ 's invariance,

$$
\mathbf{S}_{n+k}^{\pi}\left(Q_{n+k}\right) \geqq\left|V_{n}\right|\left\{Q(f)-\int p_{n}(\mathbf{x}, f) \pi(d \mathbf{x})\right\} .
$$

Divide by $\left|V_{n+k}\right|$ and let $n \rightarrow \infty$ to get

$$
\mathbf{s}^{\pi}(Q) \geqq Q(f)-p(\pi, f)=\eta(f)-p(\pi, f) .
$$

Since $k$ and $f \in \mathscr{C}_{k}$ were arbitrary, $\mathbf{s}^{\pi}(Q) \geqq p^{*}(\pi, \eta)$. By (5.26)

$$
p^{*}(\pi, \eta) \geqq \frac{1}{\left|V_{k}\right|}\left\{Q\left(\left|V_{k}\right| f\right)-\int \log \lambda^{\mathbf{x}}\left(e^{\left|V_{k}\right| f}\right) \pi(d \mathbf{x})\right\}
$$

for $f \in \mathscr{C}_{k}$, whence $p^{*}(\pi, \eta) \geqq\left|V_{k}\right|^{-1} \mathbf{S}_{k}^{\pi}\left(Q_{k}\right)$, and since $k$ was arbitrary, $p^{*}(\pi, \eta)$ $\geqq \mathbf{s}^{\pi}(Q)$.

(b) The functional $f \mapsto p(\pi, f)$ is continuous in the norm topology of $\overline{\mathscr{C}}$ and convex. By [DuS, V.3.13] the sets $\{f \in \overline{\mathscr{C}}: p(\pi, f) \leqq b\}$ are weakly closed for real $b$, which says that $f \mapsto p(\pi, f)$ is weakly lower semicontinuous. Thus $p(\pi, \cdot)$ is equal to its double dual [ET, Proposition I.4.1] and this, in light of part (a), is equivalent to part (b).

(c) Compare (5.24) and part (b).

Proof of Theorem 5.6. Define $\wp^{\mathbf{x}}:=p(\mathbf{x},-\varphi)$ and $\wp^{\pi}:=p(\pi,-\varphi)$. Boundedness of $\wp^{\mathbf{x}}$ and $\wp^{\pi}$ is trivial.

(i) By (5.14)-(5.15),

$$
\sup _{\mathbf{x} \in \mathbf{X}} \sup _{\sigma \in \Omega}\left|p_{n}(\mathbf{x},-\varphi)-\frac{1}{\left|V_{n}\right|} \log Z_{n}^{\sigma, \mathbf{x}}\right| \leqq 3 \frac{c_{r, n}}{\left|V_{n}\right|} .
$$

Letting first $n \rightarrow \infty$ and then $r \rightarrow \infty$ gives (5.7) and proves part (i).

(iii) (5.9) holds by definition, and Lemma 5.25(c) gives the statement about $\wp^{\mathrm{x}}=$ $\wp^{\pi}$. (5.5) and Lemma 5.25(b) give (5.10).

(ii) Integrating over (5.29) gives (5.8). Affinity of $\pi \rightarrow \wp^{\pi}$ is trivial. It remains to prove its upper semicontinuity.

Claim. If $\Lambda \subset \subset \mathbb{Z}^{d}, b$ is a real number and $A$ is a compact subset of $\mathscr{M}_{1}(\mathbf{X})$, then the set $B:=\bigcup_{\pi \in A}\left\{q \in \mathscr{M}_{1}\left(\boldsymbol{\Omega}_{\Lambda}\right): \mathbf{S}_{\Lambda}^{\pi}(q) \leqq b\right\}$ is compact. 
To prove the Claim, let $\left\{q_{n}\right\}$ be a sequence in $B$, with $\pi_{n} \in A$ so that $\mathbf{S}_{\Lambda}^{\pi_{n}}\left(q_{n}\right) \leqq$ $b$. By passing to a subsequence we may assume $\pi_{n} \rightarrow \pi$. Let $\mu_{n}:=\lambda_{\Lambda}^{\mathbf{x}}\left(d \sigma_{\Lambda}\right) \pi_{n}(d \mathbf{x})$, so that $\mu_{n} \rightarrow \mu:=\lambda_{\Lambda}^{\mathbf{x}}\left(d \sigma_{\Lambda}\right) \pi(d \mathbf{x})$. Let $M>0$ and pick a compact subset $K$ of $\boldsymbol{\Omega}_{\Lambda}$ such that $\mu_{n}\left(K^{c}\right)<e^{-M}$ for all $n$. Since $\mathbf{S}_{\Lambda}^{\pi_{n}}\left(q_{n}\right) \geqq H\left(q_{n} \mid \mu_{n}\right)$, taking $f=M \cdot \mathbf{1}_{K^{c}}$ in (3.2) gives

$$
M q_{n}\left(K^{c}\right) \leqq \log \mu_{n}\left(e^{M \cdot 1_{K^{c}}}\right)+b \leqq \log 2+b .
$$

Since $M$ was arbitrary, this shows that $\left\{q_{n}\right\}$ is tight. Suppose $q_{n} \rightarrow q$, by passing to a subsequence if necessary. The variational formula

$$
\mathbf{S}_{\Lambda}^{\pi}(q)=\sup _{f \in C_{b}\left(\boldsymbol{\Omega}_{\Lambda}\right)}\left\{q(f)-\int \log \lambda_{\Lambda}^{\mathbf{x}}\left(e^{f}\right) \pi(d \mathbf{x})\right\}
$$

shows that $\mathbf{S}_{\Lambda}^{\pi}(q)$ is jointly lower semicontinuous in the variable $(\pi, q)$, and hence $\mathbf{S}_{\Lambda}^{\pi}(q) \leqq \lim _{n \rightarrow \infty} \inf _{\Lambda}^{\pi_{n}}\left(q_{n}\right) \leqq b$, implying that $q \in B$. This proves the Claim.

Now we prove that, if $\pi_{n} \rightarrow \pi$, then $\wp^{\pi} \geqq \lim \sup _{n \rightarrow \infty} \wp^{\pi_{n}}$. Pass to a subsequence, again denoted by $n$, such that $\wp^{\pi_{n}}$ converges to the lim sup. Using (5.10), pick $Q_{n}$ so that $Q_{n}(\varphi)+\mathbf{s}^{\pi_{n}}\left(Q_{n}\right) \leqq-\wp^{\pi_{n}}+1 / n$. Then $\mathbf{s}^{\pi_{n}}\left(Q_{n}\right) \leqq C$ for some constant $C$, and by $(5.2) \mathbf{S}_{\Lambda}^{\pi_{n}}\left(\left(Q_{n}\right)_{\Lambda}\right) \leqq|\Lambda| C$ for all $\Lambda$. Applying the Claim to $\Lambda=V_{m}$ for all $m$ and a diagonal argument give a subsequence $Q_{n_{j}}$ that converges to a measure $Q$. Equation (5.2) shows that $\mathbf{s}^{\pi}(Q)$ is jointly lower semicontinuous in the variable $(\pi, Q)$. Thus we get

$$
\begin{aligned}
& \wp^{\pi} \geqq-Q(\varphi)-\mathbf{s}^{\pi}(Q) \geqq-\lim _{j \rightarrow \infty} Q_{n_{j}}(\varphi)-\lim _{j \rightarrow \infty} \inf ^{\pi^{\pi_{n}}}\left(Q_{n_{j}}\right) \\
& =\limsup _{j \rightarrow \infty}\left[-Q_{n_{j}}(\varphi)-\mathbf{s}^{\pi_{n_{j}}}\left(Q_{n_{j}}\right)\right] \geqq \lim _{j \rightarrow \infty} \sup \left[\wp^{\pi_{n_{j}}}-1 / n_{J}\right]=\lim _{n \rightarrow \infty} \sup _{n} \wp^{\pi_{n}} .
\end{aligned}
$$

This completes the proof of part (ii) and of the theorem.

Proof of Proposition 5.12. For each $m \in \mathbb{N}$, define $\eta_{m} \in\left(\mathscr{C}_{m}\right)^{*}$ by $\eta_{m}(g)=$ $-\xi\left(\boldsymbol{\Psi}_{g}^{V_{m}}\right)$, where the potential $\boldsymbol{\Psi}_{g}^{V_{m}} \in \mathbb{B}$ is defined as on p. 7 of [Is]. If $\psi$ is the function associated to this potential as in (2.2), then $\psi=\mathbf{R}_{m}(g)$ and consequently $\wp^{\pi}\left(\boldsymbol{\Psi}_{g}^{V_{m}}\right)=p\left(\pi,-\mathbf{R}_{m}(g)\right)=p(\pi,-g)$. By (5.13) and (5.27),

$$
-\eta_{m}(g) \leqq \frac{1}{\left|V_{m}\right|} \log \lambda\left(e^{-\left|V_{m}\right| g}\right)+C
$$

By the proof of Lemma 5.25(a) and the arguments in [Is, pp. 34-35] there is a $Q \in \mathscr{M}_{\boldsymbol{\Theta}}(\boldsymbol{\Omega})$ satisfying the conclusion of the proposition. $Q$ has $\mathbf{X}$-marginal $\pi$ because $p(\pi,-g)=-\pi(g)$ for $g \in C_{b}(\mathbf{X})$.

\section{Infinite Volume Gibbs States}

We look at various definitions of infinite volume Gibbs states for the skew and sample models. The first approach is to focus on a fixed quenched variable $\mathbf{x}$ : We let the class $\mathscr{G}^{\mathbf{x}}$ consist of those $\gamma \in \mathscr{M}_{1}(\Omega)$ that satisfy $G_{\Lambda}^{\mathbf{x}} \gamma=\gamma$ for all $\Lambda \subset \subset \mathbb{Z}^{d}$, or equivalently, for which $G_{\Lambda}^{\sigma, \mathbf{x}}$ is a version of the conditional probability $\gamma\left\{\cdot \mid \sigma_{\Lambda^{c}}\right\}$. From the observation 


$$
\int G_{\Lambda}^{\sigma, \theta_{\mathbf{i}} \mathbf{x}}(f) \gamma \circ \theta_{-\mathbf{i}}(d \sigma)=\int G_{\mathbf{i}+\Lambda}^{\sigma, \mathbf{x}}\left(f \circ \theta_{\mathbf{i}}\right) \gamma(d \sigma)
$$

it follows that $\mathscr{G}^{\mathbf{x}} \circ \theta_{-\mathbf{i}}=\mathscr{G}^{\theta_{\mathbf{i}} \mathbf{x}}$. With the continuity built into our model it is not hard to prove

\subsection{Lemma.}

(i) For all $\mathbf{x} \in \mathbf{X}, \mathscr{G}^{\mathbf{x}}$ is a nonempty, compact, convex subset of $\mathscr{M}_{1}(\Omega)$.

(ii) The set $\bigcup_{\mathbf{x} \in M} \mathscr{G}^{\mathbf{x}}$ is compact for compact $M \subset \mathbf{X}$.

(iii) The graph $\left\{(\mathbf{x}, \gamma): \gamma \in \mathscr{G}^{\mathbf{x}}\right\}$ is closed in $\mathbf{X} \times \mathscr{M}_{1}(\Omega)$.

However, the individual classes $\mathscr{G}^{\mathbf{x}}$ will not be very useful for us, as our whole approach is based on utilizing asymptotic properties that involve ergodic averaging over $\mathbf{X}$. The next step is to consider families $\left\{\gamma^{\mathbf{X}}\right\}$ of Gibbs measures, with measurable dependence on $\mathbf{x}$. We shall write $\varrho^{\cdot}$ for a measurable map $\mathbf{x} \mapsto \varrho^{\mathbf{x}}$ from $\mathbf{X}$ into $\mathscr{M}_{1}(\Omega)$, and let $\mathscr{M}^{\bullet}$ denote the space of all such maps, or measurable families of measures.

Shifts and Gibbs kernels act on such families in the obvious way, pointwise: For $\mathbf{i} \in \mathbb{Z}^{d}$ and $\Lambda \subset \subset \mathbb{Z}^{d}$, affine transformations $\theta_{\mathbf{i}}$ and $G_{\Lambda}$ on $\mathscr{M}^{\cdot}$ are defined by $\left(\theta_{\mathbf{i}} \varrho^{\bullet}\right)^{\mathbf{x}}=\varrho^{\theta_{\mathbf{i}} \mathbf{x}} \circ \theta_{\mathbf{i}}$ and $\left(G_{\Lambda} \varrho^{\bullet}\right)^{\mathbf{x}}=G_{\Lambda}^{\mathbf{x}} \varrho^{\mathbf{x}}$. Let

$$
\mathscr{M}_{\Theta}:=\left\{\varrho^{\bullet}: \theta_{\mathbf{i}} \varrho^{\bullet}=\varrho^{\bullet} \text { for all } \mathbf{i} \in \mathbb{Z}^{d}\right\}
$$

be the class of invariant families,

$$
\mathscr{G}^{\bullet}:=\left\{\varrho^{\bullet}: G_{\Lambda} \varrho^{\bullet}=\varrho^{\bullet} \text { for all } \Lambda \subset \subset \mathbb{Z}^{d}\right\}
$$

the class of Gibbs families, and $\mathscr{G}_{\Theta}^{\cdot}:=\mathscr{G}^{\bullet} \cap \mathscr{M}_{\Theta}^{\cdot}$ the class of invariant Gibbs families. Note that $\varrho^{\cdot} \in \mathscr{M}_{\Theta}^{\cdot}$ if and only if $\varrho^{\theta_{\mathbf{i}} \mathbf{x}}=\varrho^{\mathbf{x}} \circ \theta_{-\mathbf{i}}$ for all $\mathbf{i}$ and $\mathbf{x}$, and $\varrho^{\cdot} \in \mathscr{G}^{\cdot}$ if and only if $\varrho^{\mathbf{x}} \in \mathscr{G}^{\mathbf{x}}$ for all $\mathbf{x}$.

It turns out that the requirement "for all $\mathbf{x}$ " is in general too stringent, hence we need definitions that overlook sets of measure zero: Given $\pi \in \mathscr{M}_{\Theta}(\mathbf{X})$, let $\mathscr{M}^{\pi, \cdot}$ be the class of $\pi$-a.s. defined families, and corresponding to the classes defined above we have the classes $\mathscr{M}_{\Theta}^{\pi, \cdot}, \mathscr{G}^{\pi, \cdot}$, and $\mathscr{G}_{\Theta}^{\pi, \cdot}$, where the relevant condition $\left(\varrho^{\theta_{\mathbf{i}} \mathbf{x}}=\right.$ $\varrho^{\mathbf{x}} \circ \theta_{-\mathbf{i}}, \varrho^{\mathbf{x}} \in \mathscr{G}^{\mathbf{x}}$, or both) is required to hold only $\pi$-a.s.

Averaging over $\mathbf{x}$ leads us from measurable families to measures on $\Omega$ and $\boldsymbol{\Omega}$. These will be our most important classes of Gibbs measures:

6.2. Definition. Given $\pi \in \mathscr{M}_{\Theta}(\mathbf{X})$, let $\mathscr{G}^{\pi}$ be the class of probability measures $P$ on $\boldsymbol{\Omega}$ that satisfy $\mathbf{G}_{\Lambda} P=P$ for all $\Lambda \subset \subset \mathbb{Z}^{d}$ and whose $\mathbf{X}$-marginal is $\pi . \mathscr{G}_{\Theta}^{\pi}:=$ $\mathscr{G}^{\pi} \cap \mathscr{M}_{\Theta}^{\pi}(\boldsymbol{\Omega})$ is the subclass of invariant measures. Let $\mathscr{G}_{\Theta}^{\pi}$ be the class of $\Omega$ marginals of elements of $\mathscr{G}_{\boldsymbol{\Theta}}^{\pi}$.

The remainder of this section is for existence results. For the classes determined by a fixed $\pi$ this is easy. For $Q \in \mathscr{M}_{1}^{\pi}(\boldsymbol{\Omega})$, let $q^{\mathbf{x}}$ be a version of the conditional distribution of $Q$ on $\Omega$, given $\mathbf{x}$. This defines an element $q^{\bullet}: \mathbf{x} \mapsto q^{\mathbf{x}}$ of $\mathscr{M}^{\pi, \cdot}$.

6.3. Lemma. Let $\pi \in \mathscr{M}_{\Theta}(\mathbf{X})$.

(i) $\mathscr{G}_{\Theta}^{\pi}$ and $\mathscr{G}_{\Theta}^{\pi}$ are nonempty, compact, convex subsets of $\mathscr{M}_{\Theta}^{\pi}(\boldsymbol{\Omega})$ and $\mathscr{M}_{\Theta}(\Omega)$, respectively.

(ii) The map $Q \mapsto q^{\cdot}$ is an affine bijective map of $\mathscr{M}_{\boldsymbol{\Theta}}^{\pi}(\boldsymbol{\Omega})$ onto $\mathscr{M}_{\Theta}^{\pi, \cdot}$. It maps $\mathscr{G}_{\Theta}^{\pi}$ bijectively onto $\mathscr{G}_{\Theta}^{\pi, \cdot}$, so in particular $\mathscr{G}_{\Theta}^{\pi, \cdot}$ is not empty. Moreover, $\mu \in \mathscr{G}_{\Theta}^{\pi}$ if and only if there exists $a \varrho^{\cdot} \in \mathscr{G}_{\Theta}^{\pi, \cdot}$ such that $\mu=\int \varrho^{\mathbf{x}} \pi(d \mathbf{x})$. 
Proof. (i) Define a sequence of probability measures on $\boldsymbol{\Omega}$ by

$$
P^{n}(f):=\int \frac{1}{\left|V_{n}\right|} \sum_{i \in V_{n}} \mathbf{G}_{n}^{0, \mathbf{x}}\left(f \circ \theta_{\mathbf{i}}\right) \pi(d \mathbf{x}) .
$$

The boundedness of the interaction implies that this sequence is tight, and it is straightforward to check that any limit point is in $\mathscr{G}_{\boldsymbol{\Theta}}^{\pi}$. Relative compactness of $\mathscr{G}_{\Theta}^{\pi}$ follows from uniform absolute continuity with respect to $\lambda$ on all $\mathscr{F}_{\Lambda}, \Lambda \subset \subset \mathbb{Z}^{d}$, closedness follows from the continuity of the condition $\mathbf{G}_{\Lambda} P=P$, and convexity is immediate. The continuity and affinity of the projection from $\boldsymbol{\Omega}$ onto $\Omega$ extend these results to $\mathscr{G}_{\Theta}^{\pi}$. The proof of part (ii) is obvious.

To prove that $\mathscr{G}^{\cdot}$ and $\mathscr{G}_{\Theta}^{\cdot}$ are nonempty appears to be less straightforward. At present we have an argument only for the former.

6.4. Lemma. The class $\mathscr{G}^{\bullet}$ is nonempty.

Proof. Let $C=\left\{(\mathbf{x}, \gamma): \gamma \in \mathscr{G}^{\mathbf{x}}\right\}$, a closed subset of $\mathbf{X} \times \mathscr{M}_{1}(\Omega)$ by Lemma 6.1 (iii). Let $g$ be the projection $g(\mathbf{x}, \gamma)=\mathbf{x}$ from $C$ onto $\mathbf{X}$. Suppose there is a Borel subset $B$ of $C$ such that $g(B)=\mathbf{X}$ and $g$ is one-to-one on $B$. By Kuratowski's theorem [Pa, Corollary I.3.3] the inverse map $\mathbf{x} \mapsto\left(\mathbf{x}, \gamma^{\mathbf{x}}\right)$ from $\mathbf{X}$ onto $B$ is measurable, where $\gamma^{\mathbf{x}}$ is the unique element of the $\mathbf{x}$-section of $B$. This gives a measurable map $\mathbf{x} \mapsto \gamma^{\mathbf{x}}$ from $\mathbf{X}$ into $\mathscr{M}_{1}(\Omega)$ such that $\gamma^{\mathbf{x}} \in \mathscr{G}^{\mathbf{x}}$ for all $\mathbf{x}$.

It remains to prove the existence of the Borel cross section $B$. If $\mathbf{X}$ is compact, then so is $C$ by Lemma 6.1(ii) and (iii), and we can apply Theorem I.4.2 from [Pa]. The general case follows by taking $X=C$ and $Y=\mathbf{X}$ in the next lemma and by using Lemma 6.1 to verify its hypothesis.

6.5. Lemma. Suppose that $X$ and $Y$ are Polish spaces and that $g: X \rightarrow Y$ is continuous and onto, with $g^{-1}(M)$ compact for each compact $M \subset Y$. Then there is a Borel subset $B$ of $X$ such that $g(B)=Y$ and $g$ is one-to-one on $B$.

Proof. The proof is a simple modification of that given in [Pa, pp. 23-24]. Let $\bar{X}$ be a compactification of $X$ under a totally bounded metric, and let $\tilde{h}: \widetilde{A} \rightarrow \bar{X}$ be a continuous function from a closed set $\widetilde{A} \subset[0,1]$ onto $\bar{X}$ given by $[\mathrm{Pa}$, Theorem I.4.1]. Set $A:=\tilde{h}^{-1}(X) \subset \widetilde{A}, h:=\tilde{h} \mid A$, and

$$
A_{n}:=\{t \in A: g(h(s)) \neq g(h(t)) \text { for all } s \in A \text { such that } s \leqq t-1 / n\} .
$$

We claim that $A \backslash A_{n}$ is closed in $A$. Suppose $t_{m} \rightarrow t$ as $m \rightarrow \infty, t \in A$, and $t_{m} \in A \backslash A_{n}$ for all $m$. For each $m$ pick $s_{m} \leqq t_{m}-1 / n$ in $A$ so that $g\left(h\left(s_{m}\right)\right)=$ $g\left(h\left(t_{m}\right)\right)$. By passing to a subsequence we may assume that $s_{m} \rightarrow s \in \widetilde{A}$ as $m \rightarrow \infty$. By continuity, $h\left(s_{m}\right) \rightarrow \tilde{h}(s) \in \bar{X}$. The set

$$
M:=\left\{g\left(h\left(t_{m}\right)\right): m \in \mathbb{N}\right\} \cup\{g(h(t))\}
$$

is compact in $Y$. So all the $h\left(s_{m}\right)$ lie in the compact subset $g^{-1}(M)$ of $X$, hence so does $\tilde{h}(s)$. In particular, $\tilde{h}(s) \in X$, and hence $s \in A$. By continuity $g(h(s))=g(h(t))$ and $s \leqq t-1 / n$, so $t \in A \backslash A_{n}$. This proves that $A \backslash A_{n}$ is closed in $A$.

It follows that there is an open set $U$ in $\widetilde{A}$ such that $A_{n}=U \cap A . \tilde{h}(U)$ is a Borel set since $U$ is an $F_{\sigma}$ and closed subsets of $\widetilde{A}$ are compact. Hence $h\left(A_{n}\right)=\tilde{h}(U) \cap X$ 
is also Borel, for $X$ is a $G_{\delta}$ in $\bar{X}$. Now $B:=\bigcap_{n} h\left(A_{n}\right)$ defines a Borel subset of $X$.

Fix $y_{0} \in Y$. The compactness of $g^{-1}\left(y_{0}\right)$ implies that

$$
t_{0}:=\inf \left\{t \in A: g(h(t))=y_{0}\right\}
$$

lies in $A$ and $g\left(h\left(t_{0}\right)\right)=y_{0}$. That $g$ is one-to-one on $B$ follows as on p. 24 of $[\mathrm{Pa}]$.

To prove $\mathscr{G}_{\Theta}^{\bullet}$ nonempty, we would like to extend this lemma to the case where $\mathbb{Z}^{d}$ acts on $X$ and $Y$ continuously and $g$ commutes with the action, and show the existence of an invariant Borel cross section. However, an attempt to use the above argument runs into trouble: Composing $g$ with the canonical projection $p: Y \rightarrow$ $Y / \mathbb{Z}^{d}$ does give a continuous map $p \circ g: X \rightarrow Y / \mathbb{Z}^{d}$ onto the orbit space, but the orbit space can have a bad topology: For example, if $Y=\{0,1\} \mathbb{Z}^{d}$, we have dense orbits and the quotient topology of the orbit space is not even Hausdorff.

\section{Large Deviations}

In this section we study the large deviations of empirical fields under Gibbs measures. The rate functions are entropies relative to random measures as in Sect. 3, but now we take the Gibbs kernels as the random measures. We start with the skew model. For $\Lambda \subset \subset \mathbb{Z}^{d}$ and $v \in \mathscr{M}_{1}\left(\boldsymbol{\Omega}_{\Lambda}\right)$,

$$
\mathbf{K}_{\Lambda}^{\pi}(v):=\widehat{H}\left(v \mid \mathbf{G}_{\Lambda}^{0, \cdot}, \pi\right),
$$

and for $Q \in \mathscr{M}_{\Theta}(\boldsymbol{\Omega})$

$$
\mathbf{k}^{\pi}(Q):=\lim _{n \rightarrow \infty} \frac{1}{\left|V_{n}\right|} \mathbf{K}_{n}^{\pi}\left(Q_{n}\right)
$$

whenever the limit exists. (Recall that $Q_{n}$ is the marginal distribution of $\left(\sigma_{V_{n}}, \mathbf{x}\right)$ under $Q$.) Set $\mathbf{k}^{\pi}(Q)=\infty$ for noninvariant $Q$.

7.3. Lemma. Let $\pi \in \mathscr{M}_{\Theta}(\mathbf{X})$. The limit (7.2) exists for all $Q \in \mathscr{M}_{\Theta}(\boldsymbol{\Omega})$ and is given by

$$
\mathbf{k}^{\pi}(Q)=\mathbf{s}^{\pi}(Q)+\mathbf{u}(Q)+\wp^{\pi} .
$$

Suppose $P \in \mathscr{G}^{\pi}$, and let $P^{\mathbf{x}}$ be a version of the conditional probability of $P$, given x. The entropy $\widehat{h}\left(Q \mid P^{\cdot}, \pi\right)$ defined as in (3.7) exists for $Q \in \mathscr{M}_{\boldsymbol{\Theta}}(\boldsymbol{\Omega})$, and the entropy $\mathbf{h}(Q \mid P)$ defined as in (3.9) exists for $Q \in \mathscr{M}_{\boldsymbol{\Theta}}^{\pi}(\boldsymbol{\Omega})$. These entropies are connected by

$$
\mathbf{k}^{\pi}(Q)=\widehat{h}\left(Q \mid P^{\cdot}, \pi\right)= \begin{cases}\mathbf{h}(Q \mid P) & \text { if } Q \in \mathscr{M}_{\boldsymbol{\Theta}}^{\pi}(\boldsymbol{\Omega}) \\ \infty & \text { otherwise }\end{cases}
$$

If $\pi$ is ergodic, $\mathscr{M}_{\boldsymbol{\Theta}}^{\pi}(\boldsymbol{\Omega})$ can be replaced by $\mathscr{M}_{\boldsymbol{\Theta}}(\boldsymbol{\Omega})$ in $(7.5)$.

7.6. Remark. By (7.4), $\mathbf{k}^{\pi}$ inherits properties of $\mathbf{s}^{\pi}$ from (3.e): The sublevel sets $\left\{\mathbf{k}^{\pi} \leqq c\right\}$ are weakly compact, $\mathbf{k}^{\pi}$ is affine on $\mathscr{M}_{\Theta}^{\pi}(\boldsymbol{\Omega})$, and on all of $\mathscr{M}_{\Theta}(\boldsymbol{\Omega})$ in case $\pi$ is ergodic. 
Proof. Let $Q \in \mathscr{M}_{\boldsymbol{\Theta}}(\boldsymbol{\Omega})$. For any $f \in \mathscr{C}_{n}$,

$$
\begin{aligned}
Q_{n}(f) & -\int \log \mathbf{G}_{n}^{0, \mathbf{x}}\left(e^{f}\right) \pi(d \boldsymbol{x}) \\
& =Q_{n}\left(f-H_{n}^{0}\right)-\int \log \lambda_{n}^{\mathbf{x}}\left(e^{f-H_{n}^{0}}\right) \pi(d \mathbf{x})+\mathbf{U}_{n}\left(Q_{n}\right)+\wp_{n}^{\pi} .
\end{aligned}
$$

Since $H_{n}^{0} \in \mathscr{C}_{n}$ too, we get by (3.3)

$$
\mathbf{K}_{n}^{\pi}\left(Q_{n}\right)=\mathbf{S}_{n}^{\pi}\left(Q_{n}\right)+\mathbf{U}_{n}\left(Q_{n}\right)+\wp_{n}^{\pi} .
$$

From this follow both the existence of the limit in (7.2) and (7.4), and then by (3.10) $\mathbf{k}^{\pi}(Q)=\infty$ whenever $Q \mathbf{x} \neq \pi$.

Now suppose $P \in \mathscr{G}^{\pi}$. By (5.14) it follows from (7.1) that

$$
\mathbf{K}_{n}^{\pi}\left(Q_{n}\right)=\widehat{H}\left(Q_{n} \mid P_{n}^{\cdot}, \pi\right)+O\left(c_{r, n}\right) .
$$

On the other hand, if $Q \in \mathscr{M}_{\boldsymbol{\Theta}}^{\pi}(\boldsymbol{\Omega})$, by (3.4)

$$
\mathbf{K}_{n}^{\pi}\left(Q_{n}\right)=H\left(Q_{n} \mid P_{n}\right)+O\left(c_{r, n}\right) .
$$

Dividing by $\left|V_{n}\right|$, letting $n \rightarrow \infty$ and then $r \rightarrow \infty$ gives (7.5) and the statements above it. If $\pi$ is ergodic the case $Q_{\mathbf{x}} \neq \pi$ is trivial, for then $Q_{\mathbf{x}} \ll \pi$ fails and consequently $H\left(Q_{n} \mid P_{n}\right)=\infty$ for all $n$.

Next is the large deviation principle for the skew model. An earlier version appeared in Theorem IV.2 of [Com2]. Weak closures and interiors are denoted by $\vec{A}$ and $A^{\circ}$, respectively.

7.8. Theorem. Suppose $\mathbf{x}$ is generic for $\pi$. Then for any Borel subset $A$ of $\mathscr{M}_{1}(\boldsymbol{\Omega})$,

$$
\begin{aligned}
-\inf _{A^{\circ}} \mathbf{k}^{\pi} & \leqq \liminf _{n \rightarrow \infty} \frac{1}{\left|V_{n}\right|} \log \inf _{\sigma} \mathbf{G}_{n}^{\sigma, \mathbf{x}}\left\{\mathbf{R}_{n} \in A\right\} \\
& \leqq \limsup _{n \rightarrow \infty} \frac{1}{\left|V_{n}\right|} \log \sup _{\sigma} \mathbf{G}_{n}^{\sigma, \mathbf{x}}\left\{\mathbf{R}_{n} \in A\right\} \\
& \leqq-\inf _{\bar{A}} \mathbf{k}^{\pi} .
\end{aligned}
$$

If $P \in \mathscr{G}^{\pi}$, the conditional distributions $P^{\mathbf{x}}\left\{\mathbf{R}_{n} \in \cdot\right\}$ satisfy a large deviation principle with rate $\mathbf{k}^{\pi}$, for $\pi$-a.e. $\mathbf{x}$.

Proof. Equation (7.9) is a consequence of (3.e) and a version of Varadhan's theorem given as Exercise 2.1.24 in [DS]. The only technical twist is that, because $\mathbf{G}_{n}^{\sigma, \mathbf{x}} \ll \lambda_{n}^{\mathbf{x}}$ only on $\widetilde{F}_{n}$, one needs to work with the $\mathscr{F}_{n}$-measurable stationary modification of $\mathbf{R}_{n}$ defined by

$$
\tilde{\mathbf{R}}_{n}=\frac{1}{\left|V_{n}\right|} \sum_{i \in V_{n}} \delta \boldsymbol{\theta}\left(\sigma^{(n)}, \mathbf{x}\right)
$$

where $\sigma^{(n)}$ is the usual periodized configuration: $\sigma_{\mathbf{i}}^{(n)}=\sigma_{\mathbf{i}}$ for $\mathbf{i} \in V_{n}$ and $\sigma_{\mathbf{i}+(2 n+1) \mathbf{j}}^{(n)}=$ $\sigma_{\mathbf{i}}^{(n)}$ for $\mathbf{i}, \mathbf{j} \in \mathbb{Z}^{d}$. Now (7.9) follows from these three steps:

(1) By the argument of Proposition 3.1 in [Or1], the large deviation principle of (3.e) works for $\tilde{\mathbf{R}}_{n}$ too.

(2) By [DS, 2.1.24], (5.10), and (7.4), the distributions 


$$
\mu_{n}(A):=z_{n}^{-1} \int_{\boldsymbol{\Omega}} \mathbf{1}_{A}\left(\tilde{\mathbf{R}}_{n}\right) e^{-\left|V_{n}\right| \tilde{\mathbf{R}}_{n}(\varphi)} d \lambda^{\mathbf{x}}
$$

on $\mathscr{M}_{1}(\boldsymbol{\Omega})$ satisfy a large deviation principle with rate $\mathbf{k}^{\pi} . z_{n}$ is the normalization factor that makes $\mu_{n}$ into a probability measure.

(3) Now (7.9) follows for $\tilde{\mathbf{R}}_{n}$ with uniform estimates of the type (5.15), and then for $\mathbf{R}_{n}$ again as in [Or1, Proposition 3.1].

For $P \in \mathscr{G}^{\pi}, P^{\mathbf{x}}\{\cdot\}=\int \mathbf{G}_{n}^{\sigma, \mathbf{x}}\{\cdot\} P^{\mathbf{x}}(d \sigma)$ for $\pi$-a.a. $\mathbf{x}$, so

$$
\inf _{\sigma} \mathbf{G}_{n}^{\sigma, \mathbf{x}}\{\cdot\} \leqq P^{\mathbf{x}}\{\cdot\} \leqq \sup _{\sigma} \mathbf{G}_{n}^{\sigma, \mathbf{x}}\{\cdot\},
$$

which shows that the last statement of the theorem follows from (7.9).

Analogous results hold for the sample model. Set

$$
k^{\pi}(\mu):=\lim _{n \rightarrow \infty} \frac{1}{\left|V_{n}\right|} \widehat{H}\left(\mu_{n} \mid G_{n}^{0, \cdot}, \pi\right)
$$

for $\mu \in \mathscr{M}_{\Theta}(\Omega)$, and $k^{\pi}(\mu)=\infty$ for noninvariant $\mu \in \mathscr{M}_{1}(\Omega)$.

7.12. Lemma. Let $\pi \in \mathscr{M}_{\Theta}(\mathbf{X})$. Then the limit in (7.11) exists for $\mu \in \mathscr{M}_{\Theta}(\Omega)$ and satisfies

$$
k^{\pi}(\mu)=s^{\pi}(\mu)+u^{\pi}(\mu)+\wp^{\pi}
$$

and

$$
k^{\pi}(\mu)=\inf _{Q_{\Omega}=\mu} \mathbf{k}^{\pi}(Q) .
$$

$k^{\pi}$ is finite on the set $\left\{s^{\pi}<\infty\right\}$, convex, and affine in case $\pi$ is ergodic. For any $\gamma^{\cdot} \in \mathscr{G}^{\pi \cdot} \cdot$

$$
k^{\pi}(\mu)=\widehat{h}\left(\mu \mid \gamma^{\cdot}, \pi\right)
$$

7.16. Theorem. Suppose $\mathbf{x}$ is generic for $\pi$. Then (7.9) continues to hold upon replacing $\mathbf{k}^{\pi}, \mathbf{G}_{n}^{\sigma, \mathbf{x}}, \mathbf{R}_{n}$, and $\mathscr{M}_{1}(\boldsymbol{\Omega})$ with $k^{\pi}, G_{n}^{\sigma, \mathbf{x}}, R_{n}$, and $\mathscr{M}_{1}(\Omega)$, respectively. Moreover, the distributions $\gamma\left\{R_{n} \in \cdot\right\}$ on $\mathscr{M}_{1}(\Omega)$ satisfy a large deviation principle with rate $k^{\pi}$, for any $\gamma \in \mathscr{G}^{\mathbf{x}}$.

7.17. Remark. $k^{\pi}(\mu)$ is not a specific entropy relative to a Gibbs state in $\mathscr{G}^{\mathbf{x}}$. For example, if $P \in \mathscr{G}_{\Theta}^{\pi}$ and $\gamma^{\mathbf{x}}$ is a conditional distribution of $P$ on $\Omega$, given $\mathbf{x}$, then $\pi$-a.s. $\gamma^{\mathbf{x}} \in \mathscr{G}^{\mathbf{x}}$ and

$$
\lim _{n \rightarrow \infty} \frac{1}{\left|V_{n}\right|} H\left(\mu_{n} \mid \gamma_{n}^{\mathbf{x}}\right)=\mathbf{h}(\mu \otimes \pi \mid P)
$$

which in general is not equal to $k^{\pi}(\mu)$. See Lemma 8.11 below.

Proofs of Lemma 7.12 and Theorem 7.16. For $\mu \in \mathscr{M}_{\Theta}(\Omega)$ set

$$
I(\mu):=\inf _{Q_{\Omega}=\mu} \mathbf{k}^{\pi}(Q)= \begin{cases}s^{\pi}(\mu)+u^{\pi}(\mu)+\wp^{\pi} & \text { if } s^{\pi}(\mu)<\infty \\ \infty & \text { otherwise }\end{cases}
$$

The second equality above comes from (5.5) and (7.4). We shall show that the limit in (7.11) exists, $I(\mu)=k^{\pi}(\mu)$, and (7.15) holds. Let $\bar{k}$ and $\underline{k}$ be the upper and lower limit, respectively, of the right-hand side of (7.11). 
By (3.5) applied to the Gibbs kernels, $\widehat{H}\left(\mu_{n} \mid G_{n}^{0,}, \pi\right) \leqq \mathbf{K}_{n}^{\pi}\left(Q_{n}\right)$ whenever $Q \in$ $\mathscr{M}_{\Theta}(\boldsymbol{\Omega})$ has $Q_{\Omega}=\mu$, so that $\bar{k} \leqq I(\mu)$. Conversely, there is a $q \in \mathscr{M}_{1}\left(\boldsymbol{\Omega}_{n}\right)$ such that $q_{\Omega_{n}}=\mu_{n}$ and

$$
\begin{aligned}
\widehat{H}\left(\mu_{n} \mid G_{n}^{0, \cdot}, \pi\right) & =\mathbf{K}_{n}^{\pi}(q) \\
& =\mathbf{S}_{n}^{\pi}(q)+\mathbf{U}_{n}(q)+\wp_{n}^{\pi} \\
& \geqq S_{n}^{\pi}\left(\mu_{n}\right)+U_{n}^{\pi}\left(\mu_{n}\right)+\wp_{n}^{\pi},
\end{aligned}
$$

where we used (4.8) and (7.7). Dividing by $\left|V_{n}\right|$ and letting $n \rightarrow \infty$ along a suitable subsequence gives $\underline{k} \geqq I(\mu)$. This shows that the limit in (7.11) exists and equals $I(\mu)$. Equation (7.15) comes from observing that

$$
\widehat{H}\left(\mu_{n} \mid \gamma^{\bullet}, \pi\right)=\widehat{H}\left(\mu_{n} \mid G_{n}^{0, \bullet}, \pi\right)+O\left(c_{r, n}\right) .
$$

The remaining properties of $k^{\pi}$ follow from (7.13) and the corresponding properties of $s^{\pi}$ stated in (3.d).

By the contraction principle [DS, Lemma 2.14], the uniform large deviation principle as in (7.9) holds for the distributions $G_{n}^{\sigma, \mathbf{x}}\left\{R_{n} \in \cdot\right\}$ with rate $I$. For any $\gamma \in \mathscr{G}^{\mathbf{x}}$, the large deviation principle follows from inequalities similar to (7.10).

\section{Variational Principles}

In this section we establish results that correspond to the Dobrushin-Lanford-Ruelle variational principles of shift-invariant interactions. We start with the skew model. A special case of the implication (i) $\Longrightarrow$ (ii) below appeared in Theorem IV.3 of [Com2].

8.1. Theorem. Let $\pi \in \mathscr{M}_{\Theta}(\mathbf{X})$ and $P \in \mathscr{M}_{\Theta}(\boldsymbol{\Omega})$. The following are equivalent:

(i) $P \in \mathscr{G}_{\boldsymbol{\Theta}}^{\pi}$.

(ii) $\mathbf{k}^{\pi}(P)=0$.

(iii) $\mathbf{u}(P)+\mathbf{s}^{\pi}(P)=-\wp^{\pi}=\inf _{Q \in \mathscr{M}_{\Theta}(\Omega)}\left\{\mathbf{u}(Q)+\mathbf{s}^{\pi}(Q)\right\}$.

Proof. (ii) $\Longleftrightarrow$ (iii) follows from (5.10) and (7.4). (i) $\Longrightarrow$ (ii) follows from (7.5). To prove (ii) $\Longrightarrow$ (i), suppose (ii). $P_{\mathbf{X}}=\pi$ by $(7.5)$, so it remains to prove that $\mathbf{G}_{\Lambda} P=P$ for all $\Lambda \subset \subset \mathbb{Z}^{d}$. It suffices to consider the sets $\Lambda=V_{\ell}$, so to get a contradiction, assume that $\mathbf{G}_{\ell} P \neq P$ for some $\ell$.

Before getting into the derivation of the contradiction, note the following technical point: Suppose $\Delta \subset A \subset \Gamma \subset \subset \mathbb{Z}^{d}$, and set $Q:=G_{\Gamma}^{0, \mathbf{x}}(d \sigma) \pi(d \mathbf{x})$, a probability measure on $\boldsymbol{\Omega}$. Let $P_{\Lambda}^{\Lambda \backslash \Lambda}, Q_{\Lambda}^{\Lambda \backslash \Lambda}$, and $\left(\mathbf{G}_{\Delta} P\right)_{\Lambda}^{\Lambda \backslash \Delta}$ be conditional distributions of $P, Q$, and $\mathbf{G}_{\Delta} P$ on $\mathscr{F}_{\Lambda}$, given $\mathscr{\mathscr { F }}_{\Lambda \backslash \Lambda}$. Then

$$
\left|\int H\left(P_{\Lambda}^{\Lambda \backslash \Delta} \mid\left(\mathbf{G}_{\Delta} P\right)_{\Lambda}^{\Lambda \backslash \Delta}\right) d P-\int H\left(P_{\Lambda}^{\Lambda \backslash \Delta} \mid Q_{\Lambda}^{\Lambda \backslash \Delta}\right) d P\right| \leqq 4 b_{\Delta, \Lambda},
$$

where

$$
b_{\Delta, \Lambda}:=\sum_{\substack{A \cap \Delta \neq \emptyset \\ A \not \subset \Lambda A}}\left\|\Phi_{A}\right\| .
$$


This follows from

$$
\left\|\log \left(\mathbf{G}_{\Delta} P\right)_{\Lambda}^{\Lambda \backslash \Lambda}\left(e^{f}\right)-\log Q_{\Lambda}^{\Lambda \backslash \Lambda}\left(e^{f}\right)\right\| \leqq 4 b_{\Delta, \Lambda},
$$

valid for all $\mathscr{F}_{\Lambda}$-measurable bounded functions $f$.

Returning to the proof, there is a $k>\ell$ and an $\eta>0$ such that

$$
H\left(P_{k} \mid\left(\mathbf{G}_{\ell} P\right)_{k}\right) \geqq \eta
$$

By the monotonicity of relative entropy, we may pick $k$ large enough so that $b_{\ell, k}<$ $\eta / 8$. Let $m$ be arbitrary, and pick $n=n_{m}$ so that $V_{n}$ is the disjoint union of the cubes $\mathbf{i}_{1}+V_{k}, \ldots, \mathbf{i}_{m^{d}}+V_{k}$ for some set $\left\{\mathbf{i}_{1}, \ldots, \mathbf{i}_{m^{d}}\right\} \subset \subset \mathbb{Z}^{d}$. For $j=1, \ldots, m^{d}$ set $\Delta_{j}:=\mathbf{i}_{j}+V_{\ell}$ and

$$
\Lambda_{j}:=\left(\mathbf{i}_{1}+V_{k}\right) \cup \cdots \cup\left(\mathbf{i}_{j}+V_{k}\right) .
$$

Use first [DS, (4.4.8)] and the fact that $P_{\Lambda_{J} \backslash \Delta_{J}}=\left(\mathbf{G}_{\Delta_{J}} P\right)_{\Lambda_{J} \backslash \Delta_{J}}$, then the monotonicity of relative entropy, and finally (8.3) shifted by $\mathbf{i}_{j}$ to get

$$
\begin{aligned}
\int H\left(P_{\Lambda_{j}}^{\Lambda_{J} \backslash \Delta_{J}} \mid\left(\mathbf{G}_{\Delta_{j}} P\right)_{\Lambda_{j}}^{\Lambda_{j} \backslash \Delta_{J}}\right) d P & =H\left(P_{\Lambda_{j}} \mid\left(\mathbf{G}_{\Delta_{j}} P\right)_{\Lambda_{j}}\right) \\
& \geqq H\left(P_{\boldsymbol{i}_{j}+V_{k}} \mid\left(\mathbf{G}_{\Delta_{j}} P\right)_{\boldsymbol{i}_{j}+V_{k}}\right) \\
& \geqq \eta
\end{aligned}
$$

Set $\Gamma=V_{n}$ and use (8.2), the choice of $k$, [DS, (4.4.8)], and the monotonicity of entropy again to get

$$
\begin{aligned}
\eta / 2 & \leqq \int H\left(P_{\Lambda_{j} \backslash \Delta_{j}}^{\Lambda_{j}} \mid Q_{\Lambda_{j}}^{\Lambda_{j} \backslash \Delta_{j}}\right) d P \\
& =H\left(P_{\Lambda_{j}} \mid Q_{\Lambda_{j}}\right)-H\left(P_{\Lambda_{j} \backslash \Delta_{j}} \mid Q_{\Lambda_{j} \backslash \Delta_{j}}\right) \\
& \leqq H\left(P_{\Lambda_{j}} \mid Q_{\Lambda_{j}}\right)-H\left(P_{\Lambda_{j-1}} \mid Q_{\Lambda_{j-1}}\right)
\end{aligned}
$$

with the very last term missing for $j=1$. Add over $j=1, \ldots, m^{d}$ and use (3.a) to get

$$
\eta m^{d} / 2 \leqq H\left(P_{n} \mid Q_{n}\right) \leqq \mathbf{K}_{n}^{\pi}\left(P_{n}\right) .
$$

Divide by $\left|V_{n}\right|$ and let $m \nearrow \infty$ to get $\eta(2 k+1)^{-d} / 2 \leqq \mathbf{k}^{\pi}(P)$, contradicting (ii).

Next we project this result to the sample model.

8.4. Theorem. Let $\pi \in \mathscr{M}_{\Theta}(\boldsymbol{X})$ and $\mu \in \mathscr{M}_{\Theta}(\Omega)$. The following are equivalent:

(i) $\mu \in \mathscr{G}_{\Theta}^{\pi}$.

(ii) $k^{\pi}(\mu)=0$.

(iii) $u^{\pi}(\mu)+s^{\pi}(\mu)=-\wp^{\pi}=\inf _{v \in \mathscr{M}_{\Theta}(\Omega)}\left\{u^{\pi}(v)+s^{\pi}(v)\right\}$.

Proof. (i) $\Longleftrightarrow$ (ii) follows from the definition of $\mathscr{G}_{\Theta}^{\pi}$, (7.14), and (i) $\Longleftrightarrow$ (ii) in Theorem 8.1. (ii) $\Longleftrightarrow$ (iii) follows from (5.10) and (7.13).

The sample large deviation principle 7.16 and variational principle 8.4 reveal the relevance of the class $\mathscr{G}_{\Theta}^{\pi}$ for quenched randomness: If $\gamma \in \mathscr{G}^{\mathbf{x}}$ for a variable $\mathbf{x}$ generic for $\pi$, the weak limits of $R_{n}$ under $\gamma$ are contained in $\mathscr{G}_{\Theta}^{\pi}$, in the sense that for any open neighborhood of the convex, compact set $\mathscr{G}_{\Theta}^{\pi}, \gamma\left\{R_{n} \in V^{c}\right\} \rightarrow 0$ exponentially fast as $n \rightarrow \infty$. In particular, if $\mathscr{G}_{\Theta}^{\pi}=\{\mu\}$, then $R_{n} \rightarrow \mu \gamma$-a.s. 
To connect our results with those of $[\mathrm{Z}]$, let us look at variational principles in terms of invariant families as defined in Sect. 6. For the remainder of this section, $\pi$ is a fixed ergodic element of $\mathscr{M}_{\Theta}(\mathbf{X})$, and $Q \in \mathscr{M}_{\Theta}^{\pi}(\boldsymbol{\Omega})$ and $q^{\cdot} \in \mathscr{M}_{\Theta}^{\pi, \cdot}$ are associated as in Lemma 6.3(ii).

8.5. Lemma. For any $\varrho^{\cdot} \in \mathscr{M}_{\Theta}^{\pi, \cdot}$, the entropy

$$
h\left(\varrho^{\bullet} \mid \lambda^{\bullet}\right):=\lim _{n \rightarrow \infty} \frac{1}{\left|V_{n}\right|} H\left(\varrho_{n}^{\mathbf{x}} \mid \lambda_{n}^{\mathbf{x}}\right)
$$

relative to the a priori family $\lambda^{\cdot}$ exists as a $\pi$-a.s. limit, independent of $\mathbf{x}$. If the limit is finite, we have convergence in $L^{1}(\pi)$ too. Moreover,

$$
h\left(q^{\bullet} \mid \lambda^{\bullet}\right)=\mathbf{h}(Q \mid \lambda)=\mathbf{s}^{\pi}(Q) \text {. }
$$

Proof. Equation (8.6) follows from subadditive ergodic theory, see [Z, Proposition 4.2] or [Se1, Theorem 3.10]. Note in particular that a nonnegative superadditive process, integrable or not, converges a.s. [Kr, Theorem 5.4]. To get (8.7), integrate (8.6) against $\pi$ and use [DS, (4.4.8)] and (3.10).

8.8. Remark. The quantity $h\left(\varrho^{\bullet} \mid \hat{\lambda}^{*}\right)$ is the negative of the entropy defined in (4.11) of $[\mathrm{Z}]$.

Next specific energy for invariant families (see Proposition 4.5 in [Z]):

8.9. Lemma. For $\varrho^{\cdot} \in \mathscr{M}_{\Theta}^{\pi, \cdot}$,

$$
u\left(\varrho^{\cdot}\right):=\lim _{n \rightarrow \infty} \frac{1}{\left|V_{n}\right|} \varrho^{\mathbf{x}}\left(H_{n}^{0, \mathbf{x}}\right)
$$

exists as a $\pi$-a.s. and $L^{1}(\pi)$ limit, and satisfies $u\left(\varrho^{\bullet}\right)=\int \varrho^{\mathbf{x}}\left(\varphi^{\mathbf{x}}\right) \pi(d \mathbf{x})$. In particular, $u\left(q^{*}\right)=\mathbf{u}(Q)$.

Proof. Use (5.15) to write

$$
\frac{1}{\left|V_{n}\right|} \varrho^{\mathbf{x}}\left(H_{n}^{0, \mathbf{x}}\right)=\frac{1}{\left|V_{n}\right|} \sum_{\mathbf{i} \in V_{n}} \varrho^{\theta_{\mathbf{i}} \mathbf{x}}\left(\varphi^{\theta_{\mathbf{i}} \mathbf{x}}\right)+O\left(\frac{c_{r, n}}{\left|V_{n}\right|}\right),
$$

then apply the multiparameter ergodic theorem and the argument following (5.17).

The last ingredient of the variational principle is entropy relative to a Gibbs family:

8.11. Lemma. For $\varrho^{\cdot} \in \mathscr{M}_{\Theta}^{\pi \cdot \cdot}$ and $\gamma^{\cdot} \in \mathscr{G}_{\Theta}^{\pi, \cdot}$, the entropy

$$
h\left(\varrho^{\bullet} \mid \gamma^{\bullet}\right):=\lim _{n \rightarrow \infty} \frac{1}{\left|V_{n}\right|} H\left(\varrho_{n}^{\mathbf{x}} \mid \gamma_{n}^{\mathbf{x}}\right)
$$

exists as a $\pi$-a.s. limit, independent of $\mathbf{x}$, and is given by

$$
h\left(\varrho^{\bullet} \mid \gamma^{\bullet}\right)=h\left(\varrho^{\bullet} \mid \lambda^{\bullet}\right)+u\left(\varrho^{\bullet}\right)+\wp^{\pi} .
$$

Also, $h\left(q^{\bullet} \mid \gamma^{\bullet}\right)=\mathbf{k}^{\pi}(Q)$. 
Proof. Since $\gamma^{\mathbf{x}}=\int G_{n}^{\sigma, \mathbf{x}} \gamma^{\mathbf{x}}(d \sigma)$ and $G_{n}^{\sigma, \mathbf{x}}=G_{n}^{0, \mathbf{x}} \cdot e^{O\left(c_{r, n}\right)}$, we have

$$
\begin{aligned}
H\left(\varrho_{n}^{\mathbf{x}} \mid \gamma_{n}^{\mathbf{x}}\right) & =H\left(\varrho_{n}^{\mathbf{x}} \mid G_{n}^{0, \mathbf{x}}\right)+O\left(c_{r, n}\right) \\
& =H\left(\varrho_{n}^{\mathbf{x}} \mid \lambda_{n}^{\mathbf{x}}\right)+\varrho^{\mathbf{x}}\left(H_{n}^{0, \mathbf{x}}\right)+\log Z_{n}^{0, \mathbf{x}}+O\left(c_{r, n}\right),
\end{aligned}
$$

with $c_{r, n}$ again defined by (5.16). Divide by $\left|V_{n}\right|$ and let $n \rightarrow \infty$, note that $\pi$-a.e. $\mathbf{x}$ is generic for $\pi$, and then let $r \rightarrow \infty$.

And now the variational principle. The equivalence (i) $\Longleftrightarrow$ (iii) below was deduced in Theorem 4.8 of [Z] for a class of unbounded interactions with a different definition of an equilibrium family.

8.12. Theorem. Let $\pi \in \mathscr{M}_{\Theta}(\mathbf{X})$ be ergodic, $\varrho^{\cdot} \in \mathscr{M}_{\Theta}^{\pi, \cdot}$, and $\gamma^{\cdot} \in \mathscr{G}_{\Theta}^{\pi, \cdot}$. The following are equivalent:

(i) $\varrho \cdot \in \mathscr{G}_{\Theta}^{\pi,} \cdot$

(ii) $h\left(\varrho^{\bullet} \mid \gamma^{\bullet}\right)=0$.

(iii) $u\left(\varrho^{\bullet}\right)+h\left(\varrho^{\bullet} \mid \lambda^{\bullet}\right)=-\wp^{\pi}=\inf _{v^{\bullet} \in \mathscr{M}_{\Theta}^{\pi \cdot}}\left\{u\left(v^{\bullet}\right)+h\left(v^{\bullet} \mid \lambda^{\bullet}\right)\right\}$.

Proof. Theorem 8.1 and the correspondence $\mathscr{M}_{\Theta}^{\pi \cdot \cdot} \leftrightarrow \mathscr{M}_{\Theta}^{\pi}(\boldsymbol{\Omega})$ of Lemma 6.3(ii).

\section{Equivalence of Ensembles for Observables}

Our next goal is the Lanford theory [La] of large deviations and equivalence of ensembles for observables. Let $\pi \in \mathscr{M}_{\Theta}(\mathbf{X})$ be fixed throughout the section.

9.1. Definition. A collection $\mathbf{g}=\left\{g_{\Lambda}: \Lambda \subset \subset \mathbb{Z}^{d}\right\}$ of continuous functions from $\boldsymbol{\Omega}$ into $\mathbb{R}^{k}$ is an observable if for some bounded continuous function $g$ from $\boldsymbol{\Omega}$ into $\mathbb{R}^{k}$,

$$
\left\|g_{\Lambda}-\sum_{\mathbf{i} \in \Lambda} g \circ \theta_{\mathbf{i}}\right\|=o(|\Lambda|) \quad \text { as }|\Lambda| \rightarrow \infty .
$$

In particular, $\mathbf{g}=\left\{g_{\Lambda}=\left(g_{\Lambda}^{1}, \ldots, g_{\Lambda}^{k}\right): \Lambda \subset \subset \mathbb{Z}^{d}\right\}$ is an observable if there are interactions $\boldsymbol{\Psi}^{j} \in \mathbb{B}$ such that

$$
g_{\Lambda}^{j}=\sum_{A \subset A} \Psi_{A}^{j}
$$

for $j=1, \ldots, k$. For we can take $g=\left(\psi^{1}, \ldots, \psi^{k}\right)$, where $\psi^{j}$ is associated to $\boldsymbol{\Psi}^{j}$ as in (2.2), and then (9.2) follows from (5.15).

To introduce an inverse temperature $\beta$ we replace the interaction $\boldsymbol{\Phi}$ by $\beta \boldsymbol{\Phi}$. $\beta$ may be any real number. The dependence on $\beta$ is indicated by a superscript: The skew Gibbs kernels $\mathbf{G}_{\Lambda}^{\beta, \sigma, \mathbf{x}}$ are defined by

$$
d \mathbf{G}_{\Lambda}^{\beta, \sigma, \mathbf{x}}=\frac{1}{Z_{\Lambda}^{\beta, \sigma, \mathbf{x}}} e^{-\beta H_{\Lambda}} d \lambda_{\Lambda}^{\mathbf{x}} \otimes \delta_{\sigma_{\Lambda^{c}}}
$$

with the obvious $Z_{\Lambda}^{\beta, \sigma, \mathbf{x}}$, the infinite volume pressure is

$$
\wp^{\beta, \pi}=\lim _{n \rightarrow \infty} \frac{1}{\left|V_{n}\right|} \int \log Z_{n}^{\beta, \sigma, \mathbf{x}} \pi(d \mathbf{x})
$$


and $\mathscr{G}_{\Theta}^{\beta, \pi}$ denotes the class of skew Gibbs measures for the interaction $\beta \boldsymbol{\Phi}$ as defined in 6.2 .

Let $\mathbf{g}$ be any observable and $g$ the function associated to it by (9.2). We begin by recording the large deviations of the observable under the a priori measure $\lambda^{\mathbf{x}}$, the microcanonical measures $\boldsymbol{\Lambda}_{n, \delta}^{e, \mathbf{x}}$, and the canonical measures $\mathbf{G}_{n}^{\beta, \sigma, \mathbf{x}}$. Microcanonical measures are defined by conditioning the a priori measure on a thin energy shell: For $e$ real, $\delta>0$, and a Borel subset $A$ of $\boldsymbol{\Omega}$,

$$
\Lambda_{n, \delta}^{e, \mathbf{x}}(A):=\lambda^{\mathbf{x}}\left\{A|| e-\left|V_{n}\right|^{-1} H_{n}^{0} \mid \leqq \delta\right\},
$$

whenever

$$
\lambda^{\mathbf{x}}\left\{\left.|e-| V_{n}\right|^{-1} H_{n}^{0} \mid \leqq \delta\right\}>0 ;
$$

otherwise set $\boldsymbol{\Lambda}_{n, \delta}^{e, \mathbf{x}}:=\lambda^{\mathbf{x}}$. Note that by (5.15) we could just as well have defined the microcanonical probabilities by conditioning on the events $\left\{\left|\mathbf{R}_{n}(\varphi)-e\right| \leqq \delta\right\}$ as in [DSZ], without affecting the limiting behavior as first $n \rightarrow \infty$ and then $\delta \rightarrow 0$.

Next the rate functions: Define the function $I_{g}: \mathbb{R}^{k} \rightarrow[0, \infty]$ by

$$
I_{g}(v):=\inf _{Q(g)=v} \mathbf{s}^{\pi}(Q) .
$$

For $v \in \mathbb{R}^{k}, \beta \in \mathbb{R}$, and $e \in \mathbb{R}$ such that $I_{\varphi}(e)<\infty$, define (imitating [La]'s notation)

$$
\eta^{m c}(v \mid e):=I_{(g, \varphi)}(v, e)-I_{\varphi}(e)
$$

and

$$
\eta^{c}(v \mid \beta):=\inf _{Q(g)=v}\left\{\beta Q(\varphi)+\mathbf{s}^{\pi}(Q)\right\}+\wp^{\beta, \pi} .
$$

For other values of $e$ set $\eta^{m c}(v \mid e)=\infty$. Equation (9.6) makes sense because $I_{(g, \varphi)}(v, e) \geqq I_{\varphi}(e)$ by $(9.5)$. Recall also the shorthand $g_{n}=g_{V_{n}}$.

9.8. Theorem. Suppose $\mathbf{x}$ is generic for $\pi$. We have the following large deviation principles for the observable:

(i) The distributions $\lambda^{\mathbf{x}}\left\{\left|V_{n}\right|^{-1} g_{n} \in \cdot\right\}$ on $\mathbb{R}^{k}$ satisfy a large deviation principle with rate $I_{g}$.

(ii) Suppose $e$ is such that $I_{\varphi}(e)<\infty$. Then for any fixed $\delta>0,(9.4)$ holds for large enough $n$. For Borel sets $A \in \mathscr{B}_{\mathbb{R}^{k}}$,

$$
\begin{aligned}
-\inf _{v \in A^{\circ}} \eta^{m c}(v \mid e) & \leqq \liminf _{\delta \rightarrow 0} \liminf _{n \rightarrow \infty} \frac{1}{\left|V_{n}\right|} \log \Lambda_{n, \delta}^{e, \mathbf{x}}\left\{\frac{g_{n}}{\left|V_{n}\right|} \in A\right\} \\
& \leqq \limsup _{\delta \rightarrow 0} \limsup _{n \rightarrow \infty} \frac{1}{\left|V_{n}\right|} \log \Lambda_{n, \delta}^{e, \mathbf{x}}\left\{\frac{g_{n}}{\left|V_{n}\right|} \in A\right\} \\
& \leqq-\inf _{v \in \bar{A}} \eta^{m c}(v \mid e) .
\end{aligned}
$$

(iii) The distributions $\mathbf{G}_{n}^{\beta, \sigma, \mathbf{x}}\left\{\left|V_{n}\right|^{-1} g_{n} \in \cdot\right\}$ on $\mathbb{R}^{k}$ satisfy a large deviation principle with rate $\eta^{c}(\cdot \mid \beta)$, uniformly in $\sigma$ as in (7.9).

Proof of the theorem and other claims follow at the end of the section. It is clear from the variational principle 8.1 and (9.7) that

$$
\left\{v: \eta^{c}(v \mid \beta)=0\right\}=\left\{P(g): P \in \mathscr{G}_{\boldsymbol{\Theta}}^{\beta, \pi}\right\} .
$$


Hence by part (iii) above, the possible limit points of $g_{n} /\left|V_{n}\right|$ under $\mathbf{G}_{n}^{\beta, \sigma, \mathbf{x}}$ are the expectations of $g$ under Gibbs measures. More precisely, if $V$ is an open neighborhood of the convex, compact set $\left\{P(g): P \in \mathscr{G}_{\boldsymbol{\Theta}}^{\beta, \pi}\right\}$ in $\mathbb{R}^{k}$, then $\mathbf{G}_{n}^{\beta, \sigma, \mathbf{x}}\left\{\left|V_{n}\right|^{-1} g_{n}\right.$ $\left.\in V^{c}\right\} \rightarrow 0$ exponentially fast as $n \rightarrow \infty$.

Let us take a closer look at the important special case $\mathbf{g}=\boldsymbol{\Phi}$, our fixed interaction. Then the rate function $I_{\varphi}$ is in duality with pressure:

$$
-\wp \wp^{\beta, \pi}=\inf _{e \in \mathbb{R}}\left\{\beta e+I_{\varphi}(e)\right\}
$$

and

$$
-I_{\varphi}(e)=\inf _{\beta \in \mathbb{R}}\left\{\beta e+\wp^{\beta, \pi}\right\} .
$$

A very precise description of the correspondence between the dual variables $\beta$ (inverse temperature) and $e$ (energy) can be given. Our model can be in one of two situations: In the degenerate case $\mathscr{G}_{\Theta}^{\beta, \pi}=\{\lambda\}$ for all $\beta \in \mathbb{R}$ (recall that $\lambda:=$ $\left.\int \lambda^{\mathbf{x}} \pi(d \mathbf{x})\right)$, and consequently $\wp^{\beta, \pi}=-\beta \lambda(\varphi)$ for all $\beta$ and $I_{\varphi}(e)=0$ for $e=\lambda(\varphi)$ and $\infty$ otherwise.

Henceforth assume we are not in the degenerate case. Then the classes $\mathscr{G}_{\boldsymbol{\Theta}}^{\beta, \pi}$ of Gibbs measures are disjoint for all distinct $\beta$, and in particular $\lambda$ is the Gibbs measure only for $\beta=0$. To each $\beta$ corresponds a unique nonempty closed interval $\left[e_{0}(\beta), e_{1}(\beta)\right]$ of compatible energy values that can be characterized in the following ways:

$$
\begin{aligned}
{\left[e_{0}(\beta), e_{1}(\beta)\right] } & =\left\{e:-\wp^{\beta, \pi}=\beta e+I_{\varphi}(e)\right\} \\
& =\left\{P(\varphi): P \in \mathscr{G}_{\boldsymbol{\Theta}}^{\beta, \pi}\right\} \\
& =\left\{e: \eta^{c}(e \mid \beta)=0\right\} .
\end{aligned}
$$

In the last formula, the rate function $\eta^{c}(e \mid \beta)$ is the one associated to $\mathbf{g}=\boldsymbol{\Phi}$. Furthermore, the intervals $\left[e_{0}(\beta), e_{1}(\beta)\right]$ are disjoint for distinct $\beta$. (But $e_{0}(\beta)=e_{1}(\beta)$ for all but countably many $\beta$.)

Turning to (9.11), $I_{\varphi}$ is obviously convex and the set $\left\{I_{\varphi}<\infty\right\}$ is a nondegenerate bounded interval that may or may not contain either of its endpoints. If $e$ is an endpoint of $\left\{I_{\varphi}<\infty\right\}$, then either $I_{\varphi}(e)=\infty$, or $I_{\varphi}(e)<\infty$ and $I_{\varphi}$ has infinite slope (negative for left, positive for right endpoint) at $e$. To each interior point $e$ of $\left\{I_{\varphi}<\infty\right\}$ corresponds a unique $\beta=\beta(e)$ such that $e_{0}(\beta) \leqq e \leqq e_{1}(\beta)$. The infimum in (9.11) is attained at this $\beta$, and $-\beta$ is the slope of the unique tangent to $I$ at $e$. The infimum in (9.5) for $v=e$ and $g=\varphi$ is attained at $Q$ if and only if $Q \in \mathscr{G}_{\Theta}^{\beta(e), \pi}$. The association $e \mapsto \beta(e)$ is decreasing because $I_{\varphi}$ is convex, with $\beta=0$ corresponding to $e=\lambda(\varphi) . I_{\varphi}$ is differentiable on the interior $\left\{I_{\varphi}<\infty\right\}^{\circ}$, and

$$
\begin{aligned}
\left\{I_{\varphi}<\infty\right\}^{\circ} & =\bigcup_{\beta \in \mathbb{R}}\left[e_{0}(\beta), e_{1}(\beta)\right] \\
& \subset\left[\int\left(\lambda^{\mathbf{x}} \text {-ess inf } \varphi\right) \pi(d \mathbf{x}), \int\left(\lambda^{\mathbf{x}} \text {-ess } \sup \varphi\right) \pi(d \mathbf{x})\right] .
\end{aligned}
$$

Also for a general observable $\mathrm{g}$, the $\beta \leftrightarrow e$ duality clarifies the relationship of the canonical and microcanonical limit points (i.e. zeroes of the rate functions): For all $\beta$, 
Disordered Lattice Systems

$$
\left\{v: \eta^{c}(v \mid \beta)=0\right\}=\bigcup_{e_{0}(\beta) \leqq e \leqq e_{1}(\beta)}\left\{v: \eta^{m c}(v \mid e)=0\right\} .
$$

This is the "equivalence of canonical and microcanonical ensembles." In particular, if $e=e_{0}(\beta)=e_{1}(\beta)$ is the unique minimizer in (9.10), then

$$
\left\{v: \eta^{m c}(v \mid e)=0\right\}=\left\{v: \eta^{c}(v \mid \beta)=0\right\},
$$

and $\left|V_{n}\right|^{-1} g_{n}$ converges towards this set exponentially fast under both microcanonical and canonical probabilities.

Before concluding this section with the proofs, let us note that all of the above took place in the context of the skew model. Recall that the quantity $u^{\pi}(\mu)$ (see Proposition 5.4) was defined as a candidate for specific energy in the sample model, its virtue being that it gave the basic variational principle. But it is not clear whether this function would be involved in a meaningful conditioning of the sample model on an energy surface.

Proof of Theorem 9.8. By (9.2) and the argument of [Or1, Proposition 3.1], it suffices to prove the theorem for the distributions of $\mathbf{R}_{n}(g)$ instead of $g_{n} /\left|V_{n}\right|$. Then the large deviation principles of (i) and (iii) follow from (3.e) and (7.9), respectively, by the contraction technique of large deviation theory [DS, Lemma 2.1.4]. Now consider (ii). Equation (9.4) for large $n$ follows from the assumption $I_{\varphi}(e)<\infty$ and part (i) applied to $\boldsymbol{\Phi}$. For such $n$,

$$
\begin{aligned}
& \frac{1}{\left|V_{n}\right|} \log \Lambda_{n, \delta}^{e, \mathbf{x}}\left\{g_{n} /\left|V_{n}\right| \in A\right\} \\
&=\frac{1}{\left|V_{n}\right|} \log \lambda^{\mathbf{x}}\left\{\left(g_{n}, H_{n}^{0}\right) /\left|V_{n}\right| \in A \times[e-\delta, e+\delta]\right\} \\
&-\frac{1}{\left|V_{n}\right|} \log \lambda^{\mathbf{x}}\left\{H_{n}^{0} /\left|V_{n}\right| \in[e-\delta, e+\delta]\right\} .
\end{aligned}
$$

Let $\varepsilon>0$. By (i) and $I_{\varphi}$ 's lower semicontinuity there exist $\delta_{\varepsilon}$ and $n_{\delta, \varepsilon}$ such that, for $\delta<\delta_{\varepsilon}$ and $n>n_{\delta, \varepsilon}$, the last term in (9.15) is within $\varepsilon$ of $-I_{\varphi}(e)$. Apply part (i) to the observable $(\mathbf{g}, \boldsymbol{\Phi})$ to let $n \rightarrow \infty$ in (9.15). Then let $\delta \rightarrow 0$ and use the fact that

$$
\lim _{\delta \rightarrow 0} \inf _{\substack{v \in \bar{A} \\|x \in e| \leqq \delta}} I_{(g, \varphi)}(v, x)=\inf _{v \in \bar{A}} I_{(g, \varphi)}(v, e)
$$

a consequence of the lower semicontinuity and compact sublevel sets of $I_{(g, \varphi)}$.

Proof of (9.10) and (9.11). By (5.10) and (9.5) for $\mathbf{g}=\boldsymbol{\Phi}$,

$$
-\wp^{\beta, \pi}=\inf _{e \in \mathbb{R}} \inf _{Q(\varphi)=e}\left\{\beta Q(\varphi)+\mathbf{s}^{\pi}(Q)\right\}=\inf _{e \in \mathbb{R}}\left\{\beta e+I_{\varphi}(e)\right\} .
$$

This is (9.10). (9.11) follows by convex duality.

9.16. Lemma. If $\lambda \in \mathscr{G}_{\boldsymbol{\Theta}}^{\beta, \pi}$ for some $\beta \neq 0$, then $\mathscr{G}_{\boldsymbol{\Theta}}^{\beta, \pi}=\{\lambda\}$ for all real $\beta$.

Proof. By assumption,

$$
\int g(\mathbf{x}) \lambda^{\mathbf{x}}(f) \pi(d \mathbf{x})=\int g(\mathbf{x}) G_{\Lambda}^{\beta, \sigma, \mathbf{x}}(f) \lambda(d \sigma, d \mathbf{x})
$$


for all $g \in C_{b}(\mathbf{X})$ and $f \in \mathscr{C}_{\Lambda}$, so we deduce that

$$
H_{\Lambda}\left(\sigma_{\Lambda}, \tilde{\sigma}_{\Lambda^{c}}, \mathbf{x}\right)=-\beta^{-1} \log Z_{\Lambda}^{\beta, \tilde{\sigma}, \mathbf{x}}
$$

$\lambda$-a.s., for all $\Lambda \subset \subset \mathbb{Z}^{d}$. It follows easily that $\lambda \in \mathscr{G}_{\boldsymbol{\Theta}}^{\beta, \pi}$ for all $\beta$.

Suppose $P \in \mathscr{G}_{\Theta}^{\beta, \pi}$ for some $\beta \neq 0$, and $P \neq \lambda$ so that $\mathbf{s}^{\pi}(P)>0$. $\left(\mathscr{G}_{\Theta}^{0, \pi}=\{\lambda\}\right.$ is immediate.) Then by the variational principle

$$
\beta P(\varphi)+\mathbf{s}^{\pi}(P)=\beta \lambda(\varphi) .
$$

Pick $\beta^{\prime}$ so that $\left(\beta-\beta^{\prime}\right) / \beta<0$. Multiply above by $\beta^{\prime} / \beta$ to get

$$
\beta^{\prime} P(\varphi)+\mathbf{s}^{\pi}(P)=\beta^{\prime} \lambda(\varphi)+\mathbf{s}^{\pi}(P) \cdot\left(\beta-\beta^{\prime}\right) / \beta<\beta^{\prime} \lambda(\varphi) .
$$

This contradicts the variational principle because $\lambda \in \mathscr{G}_{\Theta}^{\beta^{\prime}, \pi}$.

9.17. Lemma. Either the classes $\mathscr{G}_{\Theta}^{\beta, \pi}$ are disjoint for distinct real $\beta$, or $\mathscr{G}_{\Theta}^{\beta, \pi}=\{\lambda\}$ for all real $\beta$ (the degenerate case).

Proof. In view of Lemma 9.16, it suffices to show that $Q \in \mathscr{G}_{\boldsymbol{\Theta}}^{\beta, \pi} \cap \mathscr{G}_{\boldsymbol{\Theta}}^{\beta^{\prime}, \pi}$ for $\beta \neq \beta^{\prime}$ forces $Q=\lambda$. As in the proof of Lemma 9.16, we deduce that

$$
H_{\Lambda}\left(\sigma_{\Lambda}, \tilde{\sigma}_{\Lambda^{c}}, \mathbf{x}\right)=-\left(\beta^{\prime}-\beta\right)^{-1} \log Z_{\Lambda}^{\beta, \tilde{\sigma}, \mathbf{x}} / Z_{\Lambda}^{\beta^{\prime}, \tilde{\sigma}, \mathbf{x}}
$$

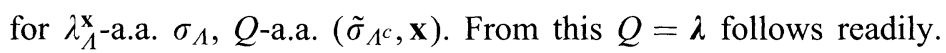

9.18. Remark. The degenerate case can take place even if $\varphi$ is not a.s. constant, with or without quenched disorder. Suppose $\mathscr{S}=\{0,1\}$, and define a function $f$ on $\mathscr{S}^{2}$ by $f(1,0)=1, f(0,1)=-1, f(0,0)=f(1,1)=0$. Then for any $\sigma \in \Omega$ and any $n$, $\sum_{1}^{n} f\left(\sigma_{i}, \sigma_{i+1}\right) \in\{-1,0,1\}$. For any measure $\mu$, the only possible rate function for the laws $\mu\left\{\frac{1}{n} \sum_{1}^{n} f \circ \theta_{i} \in \cdot\right\}$ is the trivial one: $I(0)=0$ and $I(t)=\infty$ for nonzero $t$. In particular, we have the degenerate case for the Gibbs measures of the interaction

$$
\Phi_{A}(\sigma)= \begin{cases}f\left(\sigma_{i}, \sigma_{l+1}\right) & \text { if } A=\{i, i+1\} \text { for some } i \\ 0 & \text { otherwise. }\end{cases}
$$

We return to the proofs of the section: That $\wp^{\beta, \pi}=-\beta \lambda(\varphi)$ in the degenerate case is immediate from the variational principle of Theorem 8.1. Equation (9.11) implies then that $I_{\varphi}(e)=0$ for $e=\lambda(\varphi)$ and $\infty$ otherwise.

Proof of (9.12). $P \mapsto P(\varphi)$ maps the compact convex set $\mathscr{G}_{\Theta}^{\beta, \pi}$ into $\mathbb{R}$, continuously and affinely. Its image is some compact interval. Define $e_{0}(\beta)$ and $e_{1}(\beta)$ so that $\left[e_{0}(\beta), e_{1}(\beta)\right]=\left\{P(\varphi): P \in \mathscr{G}_{\boldsymbol{\Theta}}^{\beta, \pi}\right\}$. By Theorem 8.1, $I_{\varphi}(P(\varphi))=\mathbf{s}^{\pi}(P)$ for every Gibbs measure $P$, hence $-\wp^{\beta, \pi}=\beta e+I_{\varphi}(e)$ for all $e \in\left[e_{0}(\beta), e_{1}(\beta)\right]$. Conversely, if $-\wp^{\beta, \pi}=\beta e+I_{\varphi}(e)$, find $Q$ (using (9.5) and the lower semicontinuity and compact sublevel sets of $\left.\mathbf{s}^{\pi}\right)$ such that $I_{\varphi}(e)=\mathbf{s}^{\pi}(Q)$ and $Q(\varphi)=e$. Then $-\wp^{\beta, \pi}=\beta Q(\varphi)+\mathbf{s}^{\pi}(Q)$, and Theorem 8.1 implies that $Q \in \mathscr{G}_{\boldsymbol{\Theta}}^{\beta, \pi}$. We have proved the first two equalities of (9.12). The last one is obvious from the definition (9.7). 
To show that the sets $\left[e_{0}(\beta), e_{1}(\beta)\right]$ are disjoint for distinct $\beta$, suppose we have $\beta \neq \beta^{\prime}, P \in \mathscr{G}_{\boldsymbol{\Theta}}^{\beta, \pi}$, and $P^{\prime} \in \mathscr{G}_{\boldsymbol{\Theta}}^{\beta^{\prime}, \pi}$ such that $P(\varphi)=P^{\prime}(\varphi)$. By the variational principle,

$$
\beta P(\varphi)+\mathbf{s}^{\pi}(P) \leqq \beta P^{\prime}(\varphi)+\mathbf{s}^{\pi}\left(P^{\prime}\right),
$$

hence $\mathbf{s}^{\pi}(P) \leqq \mathbf{s}^{\pi}\left(P^{\prime}\right)$, and by symmetry $\mathbf{s}^{\pi}(P)=\mathbf{s}^{\pi}\left(P^{\prime}\right)$. But then Theorem 8.1 again implies $P^{\prime} \in \mathscr{G}_{\Theta}^{\beta, \pi}$, and by Lemma 9.17 we must be in the degenerate case, which by assumption we are not.

The set $\left\{I_{\varphi}<\infty\right\}$ is an interval by the convexity of $I_{\varphi}$, but not a singleton because we have excluded the degenerate case. For all $\beta$,

$$
-\int \lambda^{\mathbf{x}} \text {-ess } \sup (\beta \varphi) \pi(d \boldsymbol{x}) \leqq \wp^{\beta, \pi} \leqq-\int \lambda^{\mathbf{x}} \text {-ess inf }(\beta \varphi) \pi(d \mathbf{x}),
$$

hence by (9.11)

$$
\left\{I_{\varphi}<\infty\right\} \subset\left[\int\left(\lambda^{\mathbf{x}} \text {-ess inf } \varphi\right) \pi(d \mathbf{x}), \int\left(\lambda^{\mathbf{x}} \text {-ess } \sup \varphi\right) \pi(d \mathbf{x})\right] .
$$

Suppose $e$ is an interior point of $\left\{I_{\varphi}<\infty\right\}$. By convexity $I_{\varphi}$ has a tangent at $e$, with slope $-\beta$ for some $\beta$. Then (9.11) forces $-I_{\varphi}(e)=\beta e+\wp^{\beta, \pi}$, so in particular $e \in\left[e_{0}(\beta), e_{1}(\beta)\right]$. If the infimum in (9.11) were achieved at distinct $\beta$ and $\beta^{\prime}$, then $e$ would be an energy value compatible with both $\beta$ and $\beta^{\prime}$, contradicting what was proved earlier. That the infimum in (9.5) for $g=\varphi$ is attained precisely on the set $\left\{Q \in \mathscr{G}_{\Theta}^{\beta(e), \pi}: Q(\varphi)=e\right\}$ is another consequence of the variational principle.

Suppose $e$ is an endpoint of $\left\{I_{\varphi}<\infty\right\}$ with $I_{\varphi}(e)<\infty$. If $I_{\varphi}$ had finite slope at $e$, then it would have infinitely many tangents at $e$ (because $I \equiv \infty$ on one side of $e$ ), again associating $e$ to multiple $\beta$, in contradiction to previous conclusions. The uniqueness of the tangent also implies that $I_{\varphi}$ is differentiable on $\left\{I_{\varphi}<\infty\right\}^{\circ}$. Equation (9.13) is evident by now.

Proof of (9.14). Suppose $\eta^{c}(v \mid \beta)=0$. Let $Q$ be a minimizer in (9.7) and $e:=Q(\varphi)$. Then

$$
0=\beta e+\inf _{\substack{Q(g)=v \\ Q(\varphi)=e}} \mathbf{s}^{\pi}(Q)+\wp^{\beta, \pi},
$$

so an application of $(9.5)$ to the observable $(\mathbf{g}, \boldsymbol{\Phi})$ gives

$$
-\wp^{\beta, \pi}=\beta e+I_{(g, \varphi)}(v, e) \text {. }
$$

Equations $(9.10),(9.19)$, and $I_{\varphi}(e) \leqq I_{(g, \varphi)}(v, e)$ combine to give $-\wp^{\beta, \pi}=\beta e+$ $I_{\varphi}(e)$ and $I_{(g, \varphi)}(v, e)=I_{\varphi}(e)$, so that $e \in\left[e_{0}(\beta), e_{1}(\beta)\right]$ and $\eta^{m c}(v \mid e)=0$.

Conversely, assume $e \in\left[e_{0}(\beta), e_{1}(\beta)\right]$ and $\eta^{m c}(v \mid e)=0$. These imply (9.19). Using (9.5), pick a $Q$ such that $Q(g, \varphi)=(v, e)$ and $I_{(g, \varphi)}(v, e)=\mathbf{s}^{\pi}(Q)$. Substitute these into (9.19) and then into (9.7) to get $\eta^{c}(v \mid \beta)=0$.

\section{Equivalence of Ensembles for Measures}

In Sect. 9 we deduced equivalence of the microcanonical and canonical ensembles by looking at the possible limit points of an observable in the infinite volume limit. In this final section of the paper we do the same for measures. We shall show that, in an appropriate sense, the limit points of the microcanonical probability measures are Gibbs measures at the temperature associated with the energy of the 
microcanonical ensemble. Our approach to this question via large deviation theory is motivated by Sect. 3 of [DSZ], where this program was carried out for invariant interactions.

For the duration of the section, fix $\pi \in \mathscr{M}_{\Theta}(\mathbf{X})$ and a real number $e$ such that $I_{\varphi}(e)<\infty$. To avoid worrying about whether $e$ is an interior point or a boundary point of $\left\{I_{\varphi}<\infty\right\}$, adopt the following convention: If $e$ is the left endpoint of $\left\{I_{\varphi}<\infty\right\}$ and $I_{\varphi}(e)<\infty$, then set $\beta(e)=\infty$ and

$$
\boldsymbol{G}_{\boldsymbol{\Theta}}^{\infty, \pi}=\left\{Q \in \mathscr{M}_{\boldsymbol{\Theta}}(\boldsymbol{\Omega}): Q(\varphi)=e, \mathbf{s}^{\pi}(Q)=I_{\varphi}(e)\right\} .
$$

Similarly for a right endpoint. Then all real numbers $e$ such that $I_{\varphi}(e)<\infty$ have a uniquely defined inverse temperature $-\infty \leqq \beta(e) \leqq \infty$ and there is a well-defined nonempty class of Gibbs measures at inverse temperature $\beta(e)$. Set

$$
\mathscr{K}:=\left\{Q \in \mathscr{G}_{\Theta}^{\beta(e), \pi}: Q(\varphi)=e\right\} .
$$

We know from Sect. 9 that $\mathscr{K}$ is the nonempty compact convex set of measures $Q$ for which $Q(\varphi)=e$ and $\mathbf{s}^{\pi}(Q)=I_{\varphi}(e)$.

Since the microcanonical measures are doubly indexed by $\delta>0$ and $n \in \mathbb{N}$, we must be precise about passing to limits. Let us say that a class $\left\{\mu_{n, \delta}\right\}$ of measures is relatively compact as first $n \rightarrow \infty$ and then $\delta \rightarrow 0$ if

(i) $\left\{\mu_{n, \delta}\right\}_{n=1}^{\infty}$ is relatively compact for each fixed $\delta$,

and

(ii) whenever $\delta_{J} \rightarrow 0$ and $v_{\delta_{J}}$ is a limit point of $\left\{\mu_{n, \delta_{j}}\right\}_{n=1}^{\infty}$ for each $j$, the sequence $\left\{v_{\delta},\right\}_{j=1}^{\infty}$ is relatively compact.

Say $v$ is a limit point of $\left\{\mu_{n, \delta}\right\}$ (as first $n \rightarrow \infty$ and then $\delta \rightarrow 0$ ) if some such sequence $\left\{v_{\delta_{j}}\right\}_{j=1}^{\infty}$ converges to $v$.

We shall first consider averaged microcanonical measures

$$
\boldsymbol{\Gamma}_{n, \delta}^{e, \mathbf{x}}:=\frac{1}{\left|V_{n}\right|} \sum_{\mathbf{i} \in V_{n}} \boldsymbol{\Lambda}_{n, \delta}^{e, \mathbf{x}} \circ \theta_{-\mathbf{i}} .
$$

Define a function $\mathbf{k}^{e}$ from $\mathscr{M}_{1}(\boldsymbol{\Omega})$ into $[0, \infty]$ by

$$
\mathbf{k}^{e}(Q):= \begin{cases}\mathbf{s}^{\pi}(Q)-I_{\varphi}(e) & \text { if } Q \text { is invariant and } Q(\varphi)=e \\ \infty & \text { otherwise. }\end{cases}
$$

10.1. Theorem. Suppose $\mathbf{x}$ is generic for $\pi$.

(i) As first $n \rightarrow \infty$ and then $\delta \rightarrow 0$, the distributions $\boldsymbol{\Lambda}_{n, \delta}^{e, \mathbf{x}}\left\{\mathbf{R}_{n} \in \cdot\right\}$ satisfy a large deviation principle with rate function $\mathbf{k}^{e}$, as in (9.9).

(ii) The probability measures $\boldsymbol{\Gamma}_{n, \delta}^{e, \mathbf{x}}$ are relatively compact as first $n \rightarrow \infty$ and then $\delta \rightarrow 0$. If $\Gamma$ is a limit point, then there is a probability measure $\alpha$ on $\mathscr{K}$ such that

$$
\Gamma=\int_{\mathscr{K}} Q \alpha(d Q)
$$

and $\alpha$ is a limit point of the laws $\boldsymbol{\Lambda}_{n, \delta}^{e, \mathbf{x}}\left\{\mathbf{R}_{n} \in \cdot\right\}$. In particular, $\Gamma$ is an element of $\mathscr{K}$.

Our techniques do not permit us to tackle individual microcanonical measures $\boldsymbol{\Lambda}_{n, \delta}^{e, \mathbf{x}}$. We can replicate Theorem 10.1 for the $\pi$-a.s. defined map $\Lambda_{n, \delta}^{e, \bullet}: \mathbf{x} \mapsto \Lambda_{n, \delta}^{e, \mathbf{x}}$, 
where $\Lambda_{n, \delta}^{e, \mathbf{x}}$ is the $\Omega$-marginal of $\Lambda_{n, \delta}^{e, \mathbf{x}}$. But to do so requires some technical assumptions and changes: Assume now that $\mathbf{X}=E^{\mathbb{Z}^{d}}$ for some Polish space $E, \mathbb{Z}^{d}$ acts on $\boldsymbol{X}$ by translations, and $\lambda_{o}^{\mathbf{x}}=\lambda_{o}^{\mathbf{x}_{\mathbf{0}}}$ depends only on the $\mathbf{x}_{\mathbf{0}}$-coordinate. Furthermore, assume $\pi$ is an i.i.d. measure on $\mathbf{X}$. Define the stationary empirical field $\mathbf{R}_{n}^{s}$ by

$$
\mathbf{R}_{n}^{s}:=\frac{1}{\left|V_{n}\right|} \sum_{\mathbf{i} \in V_{n}} \delta_{\theta \mathbf{i}}\left(\sigma^{(n)}, \mathbf{x}^{(n)}\right),
$$

where $\sigma^{(n)}$ and $\mathbf{x}^{(n)}$ are the periodized configurations defined as in the proof of Theorem 7.9. Redefine the microcanonical probability measure by

$$
\boldsymbol{\Lambda}_{n, \delta}^{e, \mathbf{x}}(A):=\lambda^{\mathbf{x}}\left\{A|| \mathbf{R}_{n}^{s}(\varphi)-e \mid \leqq \delta\right\} .
$$

The advantage of this definition is that the conditioning event now depends only on $\left(\sigma_{\mathbf{i}}, \mathbf{x}_{\mathbf{i}}: \mathbf{i} \in V_{n}\right)$.

Consider $\Lambda_{n, \delta}^{e, \cdot}$ as an element of the space $\mathscr{M}^{\pi, \cdot}$ of $\pi$-a.s. defined measurable maps from $\mathbf{X}$ into $\mathscr{M}_{1}(\Omega)$, introduced in Sect. 6. Give this space a Polish topology by identifying a map $\varrho^{\cdot}$ with the measure $\varrho^{\mathbf{x}}(d \sigma) \pi(d \mathbf{x})$ on $\boldsymbol{\Omega}$. In other words, $\varrho_{n}^{\cdot} \rightarrow \varrho^{\cdot}$ in $\mathscr{M}^{\pi \cdot \cdot}$ as $n \rightarrow \infty$ if

$$
\lim _{n \rightarrow \infty} \int \varrho_{n}^{\mathbf{x}}\left(f^{\mathbf{x}}\right) \pi(d \mathbf{x})=\int \varrho^{\mathbf{x}}\left(f^{\mathbf{x}}\right) \pi(d \mathbf{x})
$$

for all bounded continuous functions $f$ on $\boldsymbol{\Omega}$, where $f^{\mathbf{x}}(\sigma):=f(\sigma, \mathbf{x})$. Set

$$
\begin{aligned}
\mathscr{K} & :=\left\{\gamma^{\cdot} \in \mathscr{G}_{\Theta}^{\pi \cdot}: \int \gamma^{\mathbf{x}}\left(\varphi^{\mathbf{x}}\right) \pi(d \mathbf{x})=e\right\} \\
& =\left\{\varrho^{\cdot} \in \mathscr{M}^{\pi \cdot}: \int \varrho^{\mathbf{x}}\left(\varphi^{\mathbf{x}}\right) \pi(d \mathbf{x})=e \text { and } h\left(\varrho^{\cdot} \mid \lambda^{\bullet}\right)=I_{\varphi}(e)\right\} .
\end{aligned}
$$

$h\left(\varrho^{\bullet} \mid \lambda^{\bullet}\right)$ is the entropy defined in (8.6). Recall the bijection $Q \mapsto q^{\bullet}$ from $\mathscr{M}_{\Theta}^{\pi}(\boldsymbol{\Omega})$ onto $\mathscr{M}_{\Theta}^{\pi, \cdot}$ of Lemma $6.3($ ii). It restricts to a homeomorphism from $\mathscr{K}$ onto $\mathscr{K}$. With these assumptions we can state our final theorem:

10.2. Theorem. The maps $\Lambda_{n, \delta}^{e, \cdot}$ are relatively compact as first $n \rightarrow \infty$ and then $\delta \rightarrow 0$. If $\varrho^{\cdot}$ is a limit point, then there is a probability measure $\alpha$ on $\mathscr{K}$ such that

$$
\varrho=\int_{\mathscr{K}} q^{\cdot} \alpha(d Q)
$$

and $\alpha$ is a limit point of the laws $\int \boldsymbol{\Lambda}_{n, \delta}^{e, \mathbf{x}}\left\{\mathbf{R}_{n} \in \cdot\right\} \pi(d \mathbf{x})$. In particular, $\varrho$ is an element of $\mathscr{K}$.

We now turn to the proofs. For $\delta \geqq 0$ and $Q \in \mathscr{M}_{1}(\boldsymbol{\Omega})$, set

$$
\begin{gathered}
m(\delta):=\inf _{|Q(\varphi)-e| \leqq \delta} \mathbf{s}^{\pi}(Q), \\
\mathscr{K}_{\delta}:=\left\{Q \in \mathscr{M}_{1}(\boldsymbol{\Omega}): \mathbf{s}^{\pi}(Q)=m(\delta),|Q(\varphi)-e| \leqq \delta\right\},
\end{gathered}
$$

and

$$
\mathbf{k}_{\delta}^{e}(Q):= \begin{cases}\mathbf{s}^{\pi}(Q)-m(\delta) & \text { if } Q \text { is invariant and }|Q(\varphi)-e| \leqq \delta \\ \infty & \text { otherwise. }\end{cases}
$$

In this new notation, $m(0)=I_{\varphi}(e), \mathscr{K}_{0}=\mathscr{K}$, and $\mathbf{k}_{0}^{e}=\mathbf{k}^{e}$. 


\subsection{Lemma.}

(i) $m(\delta)$ is a continuous, convex, decreasing function from $[0, \infty)$ onto $\left[0, I_{\varphi}(e)\right]$.

(ii) The sets $\mathscr{K}_{\delta}$ are nonempty, convex, and compact, and if $G$ is any open neighborhood of $\mathscr{K}_{\delta}$, then $\mathscr{K}_{\delta^{\prime}} \subset G$ for $\delta^{\prime}$ close enough to $\delta$. Moreover, the union $\mathscr{K}_{0, \infty}:=\bigcup_{\delta \geq 0} \mathscr{K}_{\delta}$ is compact.

(iii) Fix $\delta>0$ and suppose $\mathbf{x}$ is generic for $\pi$. Then the distributions $\boldsymbol{\Lambda}_{n, \delta}^{e, \mathbf{x}}\left\{\mathbf{R}_{n}\right.$ $\in \cdot\}$ satisfy a large deviation principle with rate $\mathbf{k}_{\delta}^{e}$, as $n \rightarrow \infty$.

(iv) Suppose $\mathbf{x}$ is generic for $\pi$. The distributions $\boldsymbol{\Lambda}_{n, \delta}^{e, \mathbf{x}}\left\{\mathbf{R}_{n} \in \cdot\right\}$ are relatively compact as first $n \rightarrow \infty$ and then $\delta \rightarrow 0$, and all the limit points are supported on $\mathscr{K}$.

Proof. (i) $m(\delta)$ is obviously convex and decreasing, and $m(\delta)=0$ for large $\delta$. To prove continuity from the right: Let $\delta_{n} \searrow \delta$. Pick $Q_{n}$ 's such that $\left|Q_{n}(\varphi)-e\right| \leqq \delta_{n}$ and $\mathbf{s}^{\pi}\left(Q_{n}\right)=m\left(\delta_{n}\right)$, using the compact sublevel sets and lower semicontinuity of $\mathbf{s}^{\pi}$. The $Q_{n}$ 's lie inside the compact set $\left\{\mathbf{s}^{\pi} \leqq m(0)\right\}$, hence we may pass to a convergent subsequence and assume $Q_{n} \rightarrow Q$. Then $|Q(\varphi)-e| \leqq \delta$, and by lower semicontinuity

$$
m(\delta) \leqq \mathbf{s}^{\pi}(Q) \leqq \liminf _{n \rightarrow \infty} \mathbf{s}^{\pi}\left(Q_{n}\right)=\liminf _{n \rightarrow \infty} m\left(\delta_{n}\right)
$$

This suffices for continuity from the right, for $m(\delta) \geqq \limsup _{n \rightarrow \infty} m\left(\delta_{n}\right)$ by the monotonicity.

Now suppose $\delta_{n} \nearrow \delta$. Pick $Q$ and $Q_{1}$ that realize the infima in the definitions of $m(\delta)$ and $m\left(\delta_{1}\right)$, respectively. Let $t_{n} \nearrow 1$ be such that $\delta_{n}=t_{n} \delta+\left(1-t_{n}\right) \delta_{1}$. By the convexity of $\mathbf{s}^{\pi}$,

$$
\limsup _{n \rightarrow \infty} m\left(\delta_{n}\right) \leqq \limsup _{n \rightarrow \infty}\left\{t_{n} \mathbf{s}^{\pi}(Q)+\left(1-t_{n}\right) \mathbf{s}^{\pi}\left(Q_{1}\right)\right\}=m(\delta) .
$$

By monotonicity again we have continuity from the left.

(ii) The first clause is true by the properties of $\mathbf{s}^{\pi}$ from (3.e). Suppose $\delta_{n} \rightarrow$ $\delta$ and $Q_{n} \in \mathscr{K}_{\delta_{n}} \backslash G$ for some open neighborhood $G$ of $\mathscr{K}_{\delta}$. As above, we may assume $Q_{n} \rightarrow Q$. Then $Q \in G^{c}$. But $s^{\pi}\left(Q_{n}\right)=m\left(\delta_{n}\right)$ for all $n$, so by $s^{\pi}$ 's lower semicontinuity and part (i), $\mathbf{s}^{\pi}(Q) \leqq m(\delta)$. And $|Q(\varphi)-e| \leqq \delta$ by the continuity of the integral $Q(\varphi)$, hence we must have $\mathbf{s}^{\pi}(Q)=m(\delta)$ and $Q \in \mathscr{K}_{\delta}$, a contradiction with $Q \in G^{c}$.

$\mathscr{K}_{0, \infty}$ is a subset of the compact set $\left\{\mathbf{s}^{\pi} \leqq m(0)\right\}$, so we need only prove closedness. Suppose $Q_{n} \in \mathscr{K}_{\delta_{n}}$ and $Q_{n} \rightarrow Q$. If the $\delta_{n}$ remain bounded, pass to a convergent subsequence $\delta_{n^{\prime}} \rightarrow \delta$, and then $Q \in \mathscr{K}_{\delta}$ by the previous paragraph. If $\delta_{n^{\prime}} \nearrow \infty$ for some subsequence $n^{\prime}$, then $Q_{n^{\prime}}=\lambda$ for large enough $n^{\prime}$ and $Q=\lambda \in$ $\mathscr{K}_{0, \infty}$.

For (iii), let $0<\eta<\delta$ and write for large $n$,

$$
\begin{aligned}
& \frac{1}{\left|V_{n}\right|} \log \lambda^{\mathbf{x}}\left\{\mathbf{R}_{n} \in A^{\circ},\left|\mathbf{R}_{n}(\varphi)-e\right|<\delta-\eta\right\}-\frac{1}{\left|V_{n}\right|} \log \lambda^{\mathbf{x}}\left\{\left|\mathbf{R}_{n}(\varphi)-e\right| \leqq \delta+\eta\right\} \\
\leqq & \frac{1}{\left|V_{n}\right|} \log \lambda_{n, \delta}^{e, \mathbf{x}}\left\{\mathbf{R}_{n} \in A\right\} \\
\leqq & \frac{1}{\left|V_{n}\right|} \log \lambda^{\mathbf{x}}\left\{\mathbf{R}_{n} \in \bar{A},\left|\mathbf{R}_{n}(\varphi)-e\right| \leqq \delta+\eta\right\}-\frac{1}{\left|V_{n}\right|} \log \lambda^{\mathbf{x}}\left\{\left|\mathbf{R}_{n}(\varphi)-e\right|<\delta-\eta\right\} .
\end{aligned}
$$


For the legitimacy of this deduce from (3.e) that

$$
\liminf _{n \rightarrow \infty} \frac{1}{\left|V_{n}\right|} \log \lambda^{\mathbf{x}}\left\{\left|\mathbf{R}_{n}(\varphi)-e\right|<\delta-\eta\right\} \geqq-m(\delta-\eta / 2)>-\infty .
$$

Let $n \rightarrow \infty$ and then $\eta \searrow 0$, and use the large deviation principle of (3.e), the lower semicontinuity and compact sublevel sets of $\mathbf{s}^{\pi}$, and the continuity of $m(\delta)$. The upper bound follows without difficulty, but the lower bound emerges in the form

$$
\liminf _{n \rightarrow \infty} \frac{1}{\left|V_{n}\right|} \log \Lambda_{n, \delta}^{e, \mathbf{x}}\left\{\mathbf{R}_{n} \in A\right\} \geqq-\lim _{\eta \searrow 0} \inf _{\substack{Q \in A^{\circ} \\|Q(\varphi)-e|<\delta-\eta}} \mathbf{s}^{\pi}(Q)+m(\delta) .
$$

Thus we need to show that

$$
\lim _{\eta \backslash 0} \inf _{\substack{Q \in A^{\circ} \\|Q(\varphi)-e|<\delta-\eta}} \mathbf{s}^{\pi}(Q) \leqq \inf _{\substack{Q \in A^{\circ} \\|Q(\varphi)-e| \leqq \delta}} \mathbf{s}^{\pi}(Q) .
$$

Let $Q^{\prime} \in A^{\circ}$ be such that $\left|Q^{\prime}(\varphi)-e\right| \leqq \delta$ and $\mathbf{s}^{\pi}\left(Q^{\prime}\right)<\infty$. (If no such $Q^{\prime}$ exists, we can stop here.) Pick $P$ such that $|P(\varphi)-e| \leqq \delta / 2$ and $\mathbf{s}^{\pi}(P)<\infty$. Such a $P$ exists because $m(\delta / 2) \leqq m(0)<\infty$ by assumption. Pick $0<t<1$ close enough to 1 so that $Q_{t}:=t Q^{\prime}+(1-t) P$ lies in $A^{\circ} .\left|Q_{t}(\varphi)-e\right| \leqq \delta(1+t) / 2<\delta-\eta$ for all small enough $\eta$, hence

$$
\lim _{\eta \backslash 0} \inf _{\substack{Q \in A^{\circ} \\|Q(\varphi)-e|<\delta-\eta}} \mathbf{s}^{\pi}(Q) \leqq \mathbf{s}^{\pi}\left(Q_{t}\right)=t \mathbf{s}^{\pi}\left(Q^{\prime}\right)+(1-t) \mathbf{s}^{\pi}(P) .
$$

Let $t \nearrow 1$ and then vary $Q^{\prime}$ for the conclusion.

(iv) By the upper bound of the large deviation principle of part (iii), $\Lambda_{n, \delta}^{e, \mathbf{x}}\left\{\mathbf{R}_{n}\right.$ $\in G\} \rightarrow 1$ as $n \rightarrow \infty$ for any neighborhood $G$ of $\mathscr{K}_{\delta}$, which implies part (i) of the definition of relative compactness and that the limit points of $\boldsymbol{\Lambda}_{n, \delta}^{e, \mathbf{x}}\left\{\mathbf{R}_{n} \in \cdot\right\}$, for a fixed $\delta>0$, are supported by $\mathscr{K}_{\delta}$ (see the remark on p. 49 of [Pa]). All the limit points for all $\delta>0$ are supported by the compact set $\mathscr{K}_{0, \infty}$, hence also part (ii) of the definition is satisfied. The last statement of (iv) follows from part (ii) since the sets $\mathscr{K}_{\delta}$ converge to $\mathscr{K}$ as $\delta \searrow 0$.

Proof of Theorem 10.1. Part (i) follows from Lemma 10.3 by letting $\delta \searrow 0$ in part (iii). Part (ii) follows from Lemma 10.3(iv) because $\Gamma_{n, \delta}^{e, \mathbf{x}}=\boldsymbol{\Lambda}_{n, \delta}^{e, \mathbf{x}}\left(\mathbf{R}_{n}(\cdot)\right)$ is the image of $\boldsymbol{\Lambda}_{n, \delta}^{e, \mathbf{x}}\left\{\mathbf{R}_{n} \in \cdot\right\}$ under the continuous map that sends a distribution on probability measures to its expectation.

Proof of Theorem 10.2. Suppose $f$ is a bounded measurable function on $\boldsymbol{\Omega}$ that depends only on $\left(\sigma_{\mathbf{i}}, \mathbf{x}_{\mathbf{i}}: \mathbf{i} \in V_{n}\right)$. Then

$$
\begin{aligned}
\int \Lambda_{n, \delta}^{e, \mathbf{x}}\left(f^{\mathbf{x}}\right) \pi(d \mathbf{x}) & =\int \boldsymbol{\Lambda}_{n, \delta}^{e, \mathbf{x}^{(n)}}(f) \pi(d \mathbf{x})=\int \boldsymbol{\Lambda}_{n, \delta}^{e, \mathbf{x}^{(n)}}\left(\mathbf{R}_{n}^{s}(f)\right) \pi(d \mathbf{x}) \\
& =\int \boldsymbol{\Lambda}_{n, \delta}^{e, \mathbf{x}}\left(\mathbf{R}_{n}^{s}(f)\right) \pi(d \mathbf{x}) .
\end{aligned}
$$

Theorem 10.2 now follows as Theorem 10.1 did because the distributions $\boldsymbol{\Lambda}_{n, \delta}^{e, \mathbf{x}}\left\{\mathbf{R}_{n}^{s} \in\right.$ $\cdot\}$ and $\Lambda_{n, \delta}^{e, \mathbf{x}}\left\{\mathbf{R}_{n} \in \cdot\right\}$ satisfy the same large deviation principle. In particular, the distributions $\Lambda_{n, \delta}^{e, \mathbf{x}}\left\{\mathbf{R}_{n}^{s} \in \cdot\right\}$ concentrate on $\mathscr{K} \pi$-a.s. as first $n \rightarrow \infty$ and then $\delta \searrow 0$, 
hence so do the distributions $\int \boldsymbol{\Lambda}_{n, \delta}^{e, \mathbf{x}}\left\{\mathbf{R}_{n}^{s} \in \cdot\right\} \pi(d \mathbf{x})$. And by (10.4), this determines the asymptotic behavior of $\Lambda_{n, \delta}^{e, \cdot}$ in the topology of $\mathscr{M}^{\pi, \cdot}$.

\section{References}

[Ah] Aharony, A.: Tetracritical points in mixed magnetic crystals. Phys. Rev. Lett. 34 590-593 (1975)

[AW] Aizenman, M., Wehr, J.: Rounding effects of quenched randomness on first-order phase transitions. Commun. Math. Phys. 130 489-528 (1990)

[BK] Bricmont, J., Kupiainen, A.: Phase transition in the 3d random field Ising model, Commun. Math. Phys. 116 539-572 (1988)

[Cog1] Cogburn, R.: The ergodic theory of Markov chains in random environments. Z. Wahrsch. verw. Geb. 66 109-128 (1984)

[Cog2] Cogburn, R.: On direct convergence and periodicity for transition probabilities of Markov chains in random environments. Ann. Probab. 18 642-654 (1990)

[Cog3] Cogburn, R.: On the central limit theorem for Markov chains in random environments. Ann. Probab. 19 587-604 (1991)

[Com1] Comets, F.: Grandes déviations pour des champs de Gibbs sur $\mathbb{Z}^{d}$. C. R. Acad. Sci. Paris, Série I 303 (1986)

[Com2] Comets, F.: Large deviation estimates for a conditional probability distribution. Applications to random interaction Gibbs measures. Probab. Theory Relat. Fields. 80 407-432 (1989)

[Cs] Csisár, I.: I-divergence geometry of probability distributions and minimization problems. Ann. Probab. 3 146-158 (1975)

[DS] Deuschel, J.-D., Stroock, D.W.: Large Deviations. San Diego: Academic Press, 1989

[DSZ] Deuschel, J.-D., Stroock, D.W., Zessin, H.: Microcanonical distributions for lattice gases. Commun. Math. Phys. 139 83-101 (1991)

[DV] Donsker, M.D., Varadhan, S.R.S.: Asymptotic evaluation of certain Markov process expectations for large time, I. Comm. Pure Appl. Math. XXVIII 1-47 (1975)

[Du] Dudley, R.M.: Real Analysis and Probability. Pacific Grove: CA Wadsworth \& Brooks/ Cole, 1989

[DuS] Dunford, N., Schwartz, J.T.: Linear Operators, Part I. New York: J. Wiley, 1988

[E] Ellis, R.S.: Entropy, Large Deviations, and Statistical Mechanics. Berlin: Springer, 1985

[ET] Ekeland, I., Temam, R.: Convex Analysis and Variational Problems. Amsterdam: NorthHolland, 1976

[FH] Fischer, K.H., Hertz, J.A.: Spin Glasses. Cambridge: Cambridge University Press, 1991

[FO] Föllmer, H., Orey, S.: Large deviations for the empirical field of a Gibbs measure. Ann. Probab. 16 961-977 (1988)

[Ge1] Georgii, H.-O.: Gibbs Measures and Phase Transitions. Berlin: W. de Gruyter, 1988

[Ge2] Georgii, H.-O.: Large deviations and maximum entropy principle for interacting random fields on $\mathbb{Z}^{d}$. Ann. Probab. 21 1845-1875 (1993)

[Gr] Gross, L.: Thermodynamics, Statistical Mechanics and Random Fields. Springer Lecture Notes in Mathematics 929 (1982)

[HEC] van Hemmen, J.L., van Enter, A.C.D., Canisius, J.: On a classical spin class model. Z. Phys. B - Condensed Matter 50 311-336 (1983)

[Is] Israel, R.B.: Convexity in the Theory of Lattice Gases. Princeton, NJ: Princeton U. Press, 1979

[Kr] Krengel, U.: Ergodic Theorems. Berlin: de Gruyter, 1985

[La] Lanford, O.E.: Entropy and Equilibrium States in Classical Statistical Mechanics. Springer Lecture Notes in Physics 20 (1973), pp.1-113

[Le] Ledrappier, F.: Pressure and variational principle for random Ising model. Commun. Math. Physics 56 297-302 (1977)

[Ol] Olla, S.: Large deviations for Gibbs random fields. Probab. Theory Relat. Fields 77 343-357 (1988)

[Or1] Orey, S.: Large deviations in ergodic theory, Seminar on Stochastic Processes, (eds) E. Cinlar, K-L. Chung, R. Getoor, Boston: Birkhäuser, 1986, pp. 195-249 
[Or2] Orey, S.: Markov chains with stochastically stationary transition probabilities. Ann. Probab. 19, 907-928 (1991)

[Pa] Parthasarathy, K.R.: Probability Measures on Metric Spaces. New York: Academic Press, 1967

[Se1] Seppäläinen, T.: Large deviations for lattice systems I. Probab. Theory Relat. Fields 96 241-260 (1993)

[Se2] Seppäläinen, T.: Large deviations for lattice systems II. Probab. Theory Relat. Fields 97 $103-112(1993)$

[Se3] Seppäläinen, T.: Large deviations for Markov chains with random transitions. Ann. Probab. 22, 713-748 (1994)

[Se4] Seppäläinen, T.: Maximum entropy principles for disordered spins. To appear in Probab. Theory Relat. Fields

[Str] Stroock, D.W.: Microcanonical distributions for one dimensional lattice gases. Proc. of 1989 Lisbon Conf. on Stoch. Analysis, Birkhäuser

[SZ] Stroock, D.W., Zeitouni, O.: Microcanonical distributions, Gibb's states, and the equivalence of ensembles. Spitzer Festschrift, ed. R. Durrett and H. Kesten, Birkhäuser

[V] Varadhan, S.R.S.: Large Deviations and Applications. Philadelphia: SIAM, 1984

[Z] Zegarlinski, B.: Interactions and pressure functionals for disordered lattice systems. Commun. Math. Phys. 139 305-339 (1991)

Communicated by T. Spencer 
